\title{
FoxA1 and FoxA2 regulate growth and cellular identity in NKX2-1-positive lung adenocarcinoma
}

Grace Orstad $^{1,2}$, Alex Jones ${ }^{1,3,5}$, Timothy J Parnell ${ }^{1,4}$, Gabriela Fort ${ }^{1,2}$, Brian Lohman ${ }^{1,4}$, Katherine L. Gillis ${ }^{1,2}$, Olaf Klingbeil $^{6}$, Klaus Kaestner ${ }^{7}$, Christopher R Vakoc ${ }^{6}$, Eric L. Snyder ${ }^{1,2,3}$

\section{Affiliations}

1. Huntsman Cancer Institute, University of Utah, Salt Lake City, Utah, USA

2. Department of Oncological Sciences, University of Utah, Salt Lake City, Utah, USA

3. Department of Pathology, University of Utah, Salt Lake City, Utah, USA

4. Bioinformatics Shared Resource, Huntsman Cancer Institute, University of Utah, Salt Lake City, Utah, USA

5. Current affiliation: $\mathrm{HCl}$ Clinical Trials Operations, Huntsman Cancer Institute, University of Utah, Salt Lake City, Utah, USA

6. Cold Spring Harbor Laboratory, Cold Spring Harbor, New York, USA

7. Department of Genetics, Perelman School of Medicine, University of Pennsylvania, Philadelphia, Pennsylvania, USA

Corresponding author: eric.snyder@hci.utah.edu

Key words: FoxA1, FoxA2, NKX2-1, lung adenocarcinoma, cellular identity 


\section{ABSTRACT}

Changes in cellular identity (also known as histologic transformation or lineage plasticity) can drive malignant progression and resistance to therapy in multiple types of cancer, including lung adenocarcinoma (LUAD). The lineage specifying transcription factors FoxA1 and FoxA2 (FoxA1/2) regulate identity in NKX2-1/TTF1-negative LUAD. However, their role in NKX2-1-positive LUAD has not been systematically investigated. We find that Foxa1/2 knockout severely impairs tumorigenesis in KRAS-driven genetically engineered mouse models and human cell lines. Foxa1/2 deletion leads to the collapse of a dual identity state, marked by co-expression of pulmonary and gastrointestinal transcriptional programs, which has been implicated in LUAD progression. Mechanistically, loss of FoxA1/2 leads to aberrant NKX2-1 activity and genomic localization, which inhibits tumorigenesis and drives alternative cellular identity programs associated with non-proliferative states. This work demonstrates that FoxA1/2 expression is a novel lineage-specific vulnerability in NKX2-1-positive LUAD and identifies mechanisms of response and resistance to targeting FoxA1/2 in this disease.

\section{SIGNIFICANCE}

Recent advances in targeted protein degradation have the potential to make transcription factor inhibition a tractable therapeutic strategy in cancer. This study shows that targeting FoxA1/2 may have a major impact on NKX2-1-positive lung adenocarcinoma, including tumors driven by currently undruggable KRAS mutants.

\section{INTRODUCTION}

The behavior of a cancer cell is initially constrained by the specific epigenetic state of its cell of origin. Changes in cancer cell identity (often referred to as lineage switching, lineage plasticity, or histological transformation) have become increasingly well characterized as a mechanism of cancer progression and acquired resistance to targeted therapies ${ }^{1-3}$. Lung cancer is the leading cause of cancer death worldwide, and lung adenocarcinoma (LUAD) is the most frequently diagnosed subtype of this disease ${ }^{4}$. In patients, LUAD exhibits significant heterogeneity in cell identity and differentiation state ${ }^{5}$; these characteristics correlate directly with prognosis, response to available therapies, and acquisition of drug resistance ${ }^{6-9}$. Although epigenetic changes have been implicated in cell identity heterogeneity, the field lacks a comprehensive understanding of factors governing LUAD cell identity and how perturbations within these networks alter malignant potential ${ }^{10-12}$. 
Previous work from our lab and others has shown that the transcription factors FoxA1, FoxA2, and NKX2-1/TTF1 coordinately regulate cellular identity in LUAD ${ }^{13-17}$. The homeodomain transcription factor NKX2-1/TTF1 is a master regulator of normal lung morphogenesis and differentiation ${ }^{18-23}$ as well as LUAD identity ${ }^{15-17}$. NKX2-1 activity is dependent on differential interactions with many transcription factors, co-activators and co-repressors in a gene-specific manner, including FoxA1 and FoxA2 in both normal and malignant lung tissue. FoxA1/2 physically interact with NKX2-1, which enhances the expression of NKX2-1 target genes that have both NKX21 and FoxA binding motifs within the promoter ${ }^{24}$. FoxA1 and FoxA2 (FoxA1/2) are closely related paralogues in the forkhead box transcription factor family ${ }^{25}$. They share many cellular roles including the establishment and maintenance of cellular identity in multiple endodermal-derived tissues ${ }^{26}$. FoxA1/2 are critical regulators of normal foregut differentiation; embryonic deletion of Foxa1/2 in the foregut endoderm blocks branching morphogenesis of the lung ${ }^{27}$, hepatic specification ${ }^{28,29}$, and pancreatogenesis ${ }^{30}$. In the adult mouse lung, FoxA1/2 and NKX2-1 are co-expressed in the tracheal epithelium, bronchiolar epithelium, and Type 2 Alveolar cells ${ }^{31,32}$.

FoxA1 and FoxA2 play context-specific roles in multiple types of cancer ${ }^{33}$. For example, FoxA1 functions as an oncogene in breast and prostate cancer by virtue of genomic amplifications and point mutations within the forkhead box DNA binding domain ${ }^{34-36}$. In a p53-proficient LUAD genetically engineered mouse model (GEMM), we have previously shown that NKX2-1 regulates FoxA1/2 function by dictating whether they bind pulmonary or gastric marker genes ${ }^{15}$. In the absence of NKX2-1, FoxA1/2 drive a gastric differentiation program, reflecting their role in normal foregut differentiation. Nkx2-1-deleted murine tumors closely resemble a subtype of human LUAD termed invasive mucinous adenocarcinoma (IMA), which also exhibits gastric differentiation ${ }^{37}$. In contrast, FoxA1/2 bind to regulatory elements of pulmonary marker genes in both human ${ }^{16,38}$ and murine ${ }^{15}$ NKX2-1-positive LUAD. FoxA1/2 and NKX2-1 exhibit extensive genomic co-localization in LUAD, suggesting they may have cooperative functions in cancer that mirror their functions in normal tissue. NKX2-1 is genomically amplified in $\sim 12 \%$ of LUAD ${ }^{39}$, and FOXA1 is frequently co-amplified with $N K X 2-1$ ( $\sim 10 \%$ of human LUAD cases in TCGA, PanCancer Atlas, data not shown). In a functional screen of nuclear receptors and their co-regulators in human LUAD cell lines, FoxA1 was identified as pro-tumorigenic in a subset of the cell lines analyzed: those with highest FOXA1 copy number or expression exhibit varied levels of dependence on the gene, whereas cell 
lines with low levels were generally FOXA1-independent ${ }^{40}$. In contrast, a subset of human LUAD downregulate FoxA2, but FoxA1 and NKX2-1 levels were not evaluated in this study ${ }^{41}$.

Despite these observations, FoxA1 and FoxA2 have not been systematically characterized in NKX2-1-positive LUAD, which comprises $\sim 75 \%$ of human LUAD cases overall ${ }^{42}$. Here we use complementary GEMMs, organoid cultures, and human cell lines to demonstrate that FoxA1/2 are critical regulators of growth and cellular identity in NKX2-1-positive LUAD.

\section{$\underline{\text { RESULTS }}$}

\section{FoxA1/2 are required for growth of NKX2-1-positive lung adenocarcinoma}

We first evaluated expression of NKX2-1, FoxA1 and FoxA2 in primary human LUAD via immunohistochemistry (IHC) on whole tumor sections and tumor microarrays ( $n=132$ independent tumors). We found that all NKX2-1 positive tumors evaluated $(n=87)$ express FoxA1 and/or FoxA2. In contrast, 17\% (8/45) of NKX2-1-negative tumors have no detectable FoxA1 or FoxA2 expression (Supplemental Figure 1A-B, p<0.0001, Fisher's Exact test). These initial observations reveal that NKX2-1 and FoxA1/2 are co-expressed in human LUAD. NKX2-1 and FoxA1/2 are also co-expressed in the LUAD GEMM driven by KRAS ${ }^{\mathrm{G} 12 \mathrm{D}}$ and deletion of Trp53 ("KP" hereafter; Figure 1A, left panels). These data suggest that there is selective pressure for NKX2-1 positive LUAD tumors to retain FoxA1/2, and that NKX2-1-positive disease may be dependent on FoxA1/2 activity. Consistent with this possibility, stochastic NKX2-1 loss typically precedes FoxA2 loss in the KP model ${ }^{14}$.

To investigate the role of FoxA1/2 in NKX2-1-positive LUAD, we developed a sequential recombination system to delete Foxa1 and/or Foxa2 in existing KRAS driven, NKX2-1 positive lung tumors in vivo. We generated Kras $^{\text {FSF-G12D/+; }}$ Trp53 $3^{\text {Frt/Frt; }}$ Rosa26 $6^{\text {FSF-CreERT2; Foxa1 }}{ }^{\text {F/F; }}$ Foxa2 ${ }^{F / F}$ mice, as well as control mice harboring conditional alleles of either Foxa $1^{30}$ or Foxa $2^{43}$ alone. In these mice, delivery of the FlpO recombinase to the lung epithelium activates oncogenic $\operatorname{Kras}^{G 12 D}{ }^{44}$, inactivates the tumor suppressor $\operatorname{Trp53^{45}}$, and induces transcription of Cre ${ }^{\mathrm{ERT} 2}$ from the Rosa26 locus ${ }^{46}$. Upon tamoxifen treatment, Cre ${ }^{\text {ERT2 }}$ enters the nucleus and deletes Foxa1 and/or Foxa2 $\left(\mathrm{KPF}_{1}, \mathrm{KPF}_{2}\right.$, or $\mathrm{KPF}_{1} \mathrm{~F}_{2}$ hereafter) specifically in LUAD cells (Supplemental Figure 1C-D). We first used this model system to determine the effect of Foxa1/2 deletion at an early timepoint in tumor development. Lung 
tumors were initiated and allowed to develop for 6 weeks before deletion of Foxa1/2 via tamoxifen treatment. Eight weeks after tumor initiation, lung tissue was collected for histopathologic evaluation (Figure 1A). Heterozygous deletion of a single copy of Foxa1 and Foxa2 $\left(\mathrm{KPF}_{1}{ }^{\mathrm{F} /+} \mathrm{F}_{2}{ }^{\mathrm{F} /+}\right.$; shortened to $\mathrm{KP}$ hereafter), as well as homozygous deletion of either Foxa1 $\left(\mathrm{KPF}_{1}\right)$ or Foxa2 $\left(\mathrm{KPF}_{2}\right)$ alone has no effect on tumor proliferation (Figure 1B) or overall tumor burden (Figure 1C). Simultaneous deletion of both Foxa1 and Foxa2 causes a significant decrease in both proliferation (7.8-fold decrease) and overall tumor burden at an early timepoint (Figure 1B-C), demonstrating that low-grade LUAD tumors are dependent on FoxA1/2. Concomitant Foxa1/2 deletion also causes a subtle but highly reproducible change in tumor morphology, characterized by smaller, more compact nuclei (Figure 1A). In contrast, we observed no specific changes in morphology after deletion of either Foxa1 or Foxa2 alone (Supplemental Figure 1D). We next wanted to discern whether Foxa1/2 deletion simply slows the rate of growth or if it causes tumor regression. Using weekly $\mu \mathrm{CT}$ scans of tumor bearing mice, we found that tamoxifen treatment caused an initial decrease in tumor burden, followed by disease stasis over the course of five weeks. In contrast, vehicle treated mice had a continuous increase in tumor burden over the same period of time (Supplemental Figure 1E).

To evaluate long term consequences of Foxa1/2 deletion, we performed a survival study in which tumors developed for 10 weeks prior to tamoxifen or vehicle injections. At this timepoint, a subset of tumors in the KP mouse model have progressed to higher grade, macroscopic lesions ${ }^{47}$. Concomitant deletion of both Foxa1 and Foxa2 in established tumors causes a substantial increase in survival, extending median lifespan by 13.5 weeks (Figure 1D). In contrast, deletion of a single allele of both Foxa1 and Foxa2 (Figure 1E), or Foxa2 alone has no impact on median overall survival, and deletion of Foxa1 alone leads to a slight (1.5 week) increase in median survival (Supplemental Figure 1F-G). We observed high levels of recombination in tamoxifen treated $\mathrm{KPF}_{1}, \mathrm{KPF}_{2}$ and $\mathrm{KPF}_{1} \mathrm{~F}_{2}$ mice, although incomplete recombinants could also be detected in most mice by IHC, particularly $\mathrm{KPF}_{1} \mathrm{~F}_{2}$ mice that survived for extended periods of time (data not shown). Foxa1/2 deletion in established tumors also increased survival in a LUAD model driven by BRAF ${ }^{\mathrm{V} 600 \mathrm{E}}\left(B R A F^{\text {FSF-V600E/+ }} ; \operatorname{Trp}^{\text {Frt/Frt }}\right)^{48}$, demonstrating that FoxA1/2 are required for the growth of LUAD harboring distinct driver mutations (Supplemental Figure $1 \mathrm{H}-\mathrm{I}$ ). Of note, we detected a higher proportion of incomplete recombinants in tamoxifen treated $B P F_{1} F_{2}$ survival mice than $\mathrm{KPF}_{1} \mathrm{~F}_{2}$ mice. This is likely due the use of lentivirus in $\mathrm{KPF}_{1} \mathrm{~F}_{2}$ mice rather than adenovirus, which may have 
led to a higher recombination rate of the Rosa26FSFCreERT2 allele. Detailed histological analysis of the survival studies can be found in Supplemental Table 1. Together, these results establish that FoxA1 and FoxA2 are necessary for in vivo growth of NKX2-1-positive LUAD.

We derived cell lines from individual macroscopic $\mathrm{KPF}_{1} \mathrm{~F}_{2}$ tumors under either standard 2D (3311) or threedimensional, Matrigel-based organoid (1027B, 1027D, and 1292B) culture conditions. Transient treatment with 4-hydroxytamoxifen (4-OHT) generates isogenic pairs of organoid and cell lines that differ solely in the presence or absence of FoxA1/2 (Figure 2A, Supplemental Figure 2A). In agreement with our in vivo data, in vitro deletion of Foxa1/2 in both cell lines and organoids leads to a significant decrease in proliferation (Figure 2B-C). In subcutaneous tumors, deletion of Foxa1/2 initially causes modest but significant tumor regression $(p<0.003)$, followed by slower growth, a pattern which closely matches in vivo MicroCT analysis (Figure 2D, see also 7G). Upon histopathologic analysis, we found that the tamoxifen treated subcutaneous tumors were predominantly FoxA1/2-negative ( 65-100\%) and identified two distinct morphologies in Foxa1/2-deleted tumors: a moderate to poorly differentiated adenocarcinoma component (similar to controls) and a distinct sarcomatoid/quasimesenchymal component, which exhibited moderately lower NKX2-1 levels (Supplemental Figure 2B-C).

We next wanted to directly examine FoxA1/2 dependence in NKX2-1-positive human LUAD cell lines by knocking out FoxA1/2 via CRISPR/Cas9. To accomplish this, we selected three cell lines harboring KRAS mutations that express NKX2-1, as well as one or both of the FoxA1/2 proteins: $\mathrm{NCl}-\mathrm{H} 358, \mathrm{NCl}-\mathrm{H} 441$, and $\mathrm{NCl}-\mathrm{H} 2009$. Cells stably expressing Cas9 were transduced with three independent lentivectors that simultaneously express unique sgRNAs targeting both FoxA1 and FoxA2 (sgFoxA\#1, sgFoxA\#10, and sgFoxA\#11), as well as a non-targeting dual sgRNA control (sgNT). The three sgRNA combinations have varied levels of efficiency in targeting FoxA1/2: sgFoxA\#1 yields the weakest decrease in FoxA1/2 protein levels, while both sgFoxA\#10 and sgFoxA\#11 reduce FoxA1/2 protein levels to a much greater extent (Figure 2E). The difference in FoxA1/2 targeting efficiency correlates directly with decrease in proliferation: the least efficient sgFoxA\#1 yields an average of $40 \%$ decrease in proliferation, while the more efficient sgFoxA\#10 and sgFoxA\#11 cause a 94\% and 90\% decrease, respectively (Figure 2F). Combined, these murine and human results demonstrate that FoxA1/2 are required for the growth of NKX2-1 positive LUAD in vivo and in vitro. 


\section{FoxA1/2 enforce GI-related and Alveolar Type 2 programs in NKX2-1-positive lung adenocarcinoma}

We next sought to determine whether FoxA1/2 regulate cellular identity programs associated with malignant progression in NKX2-1-positive LUAD. We have shown previously that FoxA1/2 are critical activators of gastric differentiation in NKX2-1-negative LUAD ${ }^{15,49}$. Recent single cell analyses of the KP LUAD GEMM, including in vivo lineage recording of cancer progression, defined multiple transcriptional programs associated with distinct cellular identities that emerge as tumors progress ${ }^{11,12,50}$. Early low grade tumor cells most closely resemble normal alveolar type 2 (AT2) cells, as evidenced by high levels of Nkx2-1 and its canonical AT2 targets. As tumors progress (12-30 weeks after initiation), subsets of higher grade LUAD cells upregulate distinct gastrointestinal (GI) transcriptional programs despite the continued expression of Nkx2-1. (For simplicity, we use the term "dual identity" to refer to cell clusters in which NKX2-1 and the AT2 program are co-expressed with nonpulmonary programs.) In late stage disease, a sub-population of cells reach a terminal poorly differentiated/ EMT-like transcriptional state that lacks both AT2 and GI differentiation. Transition through dual identity states is a key component of malignant progression, but the mechanisms driving these lineage changes are not fully defined.

In order to determine which cellular identities are regulated by FoxA1/2 in NKX2-1-positive LUAD, we performed scRNAseq on in vivo $\mathrm{KPF}_{1} \mathrm{~F}_{2}$ and $\mathrm{KP}$ tumors initiated with Ad5mSPC-FlpO (to specifically target SPC-positive epithelial cells). Mice were injected with tamoxifen 10 weeks post tumor-initiation. Twelve weeks after tumor initiation, we macrodissected individual tumors, digested into single cell suspensions, depleted CD45+ and CD31+ cells via magnetic activated cell sorting (MACS) to increase representation of tumor cells, and sequenced cells both pre-depletion and post-depletion ( $n=2$ mice per genotype, multiple tumors per mouse). We visualized single cells in reduced dimensionality using uniform manifold approximation and projection (UMAP) and identified 25 major clusters between both genotypes (Supplemental Figure 3A). Of the 25 clusters, 10 were identified as tumor cells based on expression of FlpO-induced Cre ERT2 transcripts and low levels of stromal specific marker genes (Supplemental Figure 3B; Supplemental Table 2). The remaining 15 clusters were identified as immune cells, endothelial cells, fibroblasts, and normal lung epithelium (Supplemental Figure 3B-C; Supplemental Table 2). Analysis of only the tumor cell population identified 13 major clusters (Figure 3A), of which six are largely KP specific (Clusters 1, 3, 4, 5, 6, and 12), four are $\mathrm{KPF}_{1} \mathrm{~F}_{2}$ specific (Clusters 2, 7, 8, and 9), and three contain cells 
from both $\mathrm{KP}$ and $\mathrm{KPF}_{1} \mathrm{~F}_{2}$ mice (Clusters 0, 10, and 11; Figure 3B, Supplemental Figure 3D-E). Upon closer inspection, we found that the cells from $\mathrm{KPF}_{1} \mathrm{~F}_{2}$ samples that cluster with the KP tumor cells (Clusters 0 , 10, and 11) are Foxa1/2-positive incomplete recombinants, based on equivalent levels of the Foxa1 exon flanked by loxP sites in both $\mathrm{KP}$ and $\mathrm{KPF}_{1} \mathrm{~F}_{2}$ cells (Figure $3 \mathrm{C}$ ). In contrast, cells in $\mathrm{KPF}_{1} \mathrm{~F}_{2}$ specific clusters appear to be complete recombinants based on much lower levels of this exon compared to KP specific clusters (Supplemental Figure 3F). The observation that multiple clusters contain either normal stromal cells or unrecombined tumor cells from mice of both genotypes suggests that cell type, rather than genotype per se, is the primary driver of clustering. Thus, the near-complete separation of FoxA1/2-negative and FoxA1/2-positive tumor cells shows that in vivo Foxa1/2 deletion has a major impact on the transcriptome of LUAD cells. To complement this in vivo analysis, we also performed bulk RNAseq on isogenic $\mathrm{KPF}_{1} \mathrm{~F}_{2}$ organoid pairs, which identified 856 differentially expressed genes: 438 enriched in FoxA1/2-positive organoids and 418 enriched in FoxA1/2-negative organoids (Supplemental Table 3).

We then intersected scRNAseq and organoid RNAseq data with the key transcriptional cell identity programs defined by Marjanovic et $\mathrm{al}^{11}$. As expected, we identified distinct cell clusters from KP mice expressing most of these programs, including AT2 (Cluster 0), AT1/AT2 (Cluster 10), endodermal/gastric (Clusters 3-6), and ‘Highly Mixed' programs (Cluster 1; Figure 3D, Supplemental Figure 3F). We also identified smaller clusters expressing the EMT (Cluster 12) and high cycling (Cluster 11) programs, which likely correspond to a small number of poorly differentiated and G2/M phase cells, respectively. Thus, KP cells exhibit the full range of identities known to arise during LUAD progression ${ }^{11}$. Importantly, multiple clusters exhibit simultaneous expression of endodermal/gastric programs with Nkx2-1 and the AT2 program (albeit at lower levels than cluster 0) (Figure 3D-E). These "dual identity" cells are also detectable by IHC for NKX2-1 and the GI marker HNF4 $\alpha$, which are co-expressed in a subset of tumor cells in KP mice at this timepoint (Figure 3G). NKX2-1/HNF4 $\alpha$ dual positive cells are also readily identified in KP organoids (Supplemental Figure 3G).

We next asked whether these programs are FoxA1/2-dependent by evaluating their levels in $\mathrm{KPF}_{1} \mathrm{~F}_{2}-\mathrm{Specific}$ clusters (i.e. those containing predominantly complete recombinants). Maintenance of non-pulmonary endodermal programs appears to be almost entirely dependent on FoxA1/2. The embryonic liver-like and gastric- 
like programs are strongly expressed in several KP-specific clusters (6, 5, 4, 3, and 1), but are significantly downregulated in the FoxA1/2-negative clusters (Figure 3D). Less than 3\% of all cells with gene module scores at or over the midpoint of the range of expression for the Embryonic Liver and Gastric transcriptional programs across all tumor cells are FoxA1/2-negative (Figure 3F). Canonical markers of gastro-intestinal identity, such as $H n f 4 \alpha, G k n 1, G k n 2, G k n 3, L g a l s 4, T f f 1$, and Vsig, are uniquely expressed in FoxA1/2-positive clusters (Figure 3E, Supplemental Figure 3H). In LUAD organoids, Foxa1/2 deletion causes an overall decrease in the expression of GI programs associated with LUAD progression (Figure $3 \mathrm{H}$ ), including a consistent and significant decrease in canonical GI marker genes (Figure 3I). At the protein level, HNF4 $\alpha$ is lost following Foxa1/2 deletion in vivo (Figure 3G) and in vitro (Supplemental Figure 3G). Together, these data show that the dual identity state, characterized by the co-expression of pulmonary and GI transcriptional programs, is critically dependent on FoxA1/2 in LUAD.

The AT2-like program is most strongly expressed in cluster 0 , which is a FoxA1/2-positive cluster. Deletion of Foxa1/2 causes an overall decrease in the AT2 program (and in Sftpc, one of the most specific AT2 cell markers) in vivo and in organoids (Figure 3D,H, Supplemental Figure 3F). However, the AT2 program is not completely lost after Foxa1/2 deletion, and there is significant heterogeneity amongst the $\mathrm{KPF}_{1} \mathrm{~F}_{2}$-specific clusters. Several AT2 genes are detectable in three of the four $\mathrm{KPF}_{1} \mathrm{~F}_{2}$-specific clusters (Clusters 2, 8, and 9) although their levels are generally lower than KP cells (Cluster 0; Supplemental Figure 3I,H). Meanwhile, there are specific genes in each cluster (such as $C x c / 15$ in Cluster 9 and Napsa in Clusters 8 and 2) that are expressed at approximately the same level as cluster 0 (Supplemental Figure 3H). Importantly, IHC for pro-SPB and pro-SPC shows that the significant decrease in Sftpb and Sftpc transcripts leads to a corresponding decrease in protein levels in $\mathrm{KPF}_{1} \mathrm{~F}_{2}$ tumors, despite the continued expression of NKX2-1 (Figure 3J). In organoid cultures, Foxa1/2 deletion yields a similar pattern: some AT2 markers significantly decrease, whereas others (e.g. Napsa) are not significantly impacted (Figure 3K). In human LUAD cell line H441, FOXA1/2 knockout also causes a significant decrease in SFTPA1 and SFTPB (Supplemental Figure 3J). Thus, FoxA1/2 are necessary for full activation of the AT2-like transcriptional program, but AT2 identity is not completely dependent on FoxA1/2 transcriptional activity in LUAD. 
Taken together, these data show that the dual identity state associated with LUAD progression undergoes near complete collapse after Foxa1/2 deletion, with only some elements of AT2 identity remaining in a subset of $\mathrm{KPF}_{1} \mathrm{~F}_{2}$ tumor cells (Figure $3 \mathrm{~L}$ ).

\section{FoxA1/2 suppress alternative pulmonary and stratified squamous transcriptional programs in}

\section{NKX2-1-positive lung adenocarcinoma}

After identifying cell identity programs dependent on FoxA1/2, we determined the transcriptional state(s) to which LUAD cells equilibrate in the absence of FoxA1/2. We noted high levels of the AT1/AT2 program in a subset of FoxA1/2-negative cells (Figure 3D-F), so we first evaluated relative levels of AT1 vs. AT2 transcriptional programs in more depth. Using gene signatures of normal AT1 and AT2 cells derived from the same study ${ }^{51}$, we found that the Krasnow AT2 signature is detectable in FoxA1/2-negative Clusters 2, 8, and 9 (Figure 4A), but at a lower level than Cluster 0 FoxA1/2-positive cells, consistent with our analysis of the Marjanovic AT2-like signature (Figure 3D-E, Supplemental Figure 3I). The Krasnow AT1 signature is readily detectable in Clusters 2 and 7 (Figure 4B), as are an AT1 signature from the LungGENS database ${ }^{52}$ (Supplemental Figure 4A) and the AT1 genes uniquely dependent on NKX2-1 as defined by Little et al ${ }^{22}$ (Figure 4C). Close inspection of Cluster 2 reveals an inverse correlation between AT2 and AT1 identities within this cluster (Supplemental Figure 4B-C). FoxA1/2-mediated inhibition of AT1 differentiation is also evident in LUAD organoids. Using Gene Set Enrichment Analysis (GSEA), we found that Foxa1/2 deletion in organoids impairs AT2 differentiation while promoting AT1 differentiation (Figure 3H, 4E).

Like the AT1/AT2-like LUAD program, the 'Highly Mixed'/Cldn4-high program from Marjanovic et al is found in both FoxA1/2-positive and FoxA1/2-negative clusters (Cluster 1 vs. 7, Figure 3 D-F). Three recent publications have described a distinct alveolar cell state that emerges as AT2 cells differentiate into AT1 cells in response to lung injury, which Strunz et $\mathrm{al}^{53}$ have termed Alveolar Differentiation Intermediate (ADI; also described by Kobayashi et $\mathrm{al}^{54}$ as PATS - pre-alveolar type- 1 transitional cell state, and Choi et al ${ }^{55}$ as DATPs damage-associated transient progenitors). They found that this intermediate alveolar cell type is not simply undergoing a gradual loss of AT2 characteristics while acquiring AT1 characteristics; rather this is a unique transitional cell population that emerges in response to injury and is marked by high Cldn4 levels and activation 
of signaling pathways such as NFKB and TGF $\beta$. The ADI and PATS signatures are both strongly expressed in a subset of KP and $\mathrm{KPF}_{1} \mathrm{~F}_{2}$ LUAD cells and overlap with the 'Highly Mixed' LUAD signature in our dataset (Figure 4D; Supplemental Figure 4D). Additionally, GSEA reveals enrichment of these gene sets in organoids upon loss of FoxA1/2 in vitro (Figure 4E). This suggests that the LUAD cells previously described as 'Highly Mixed' may have activated the transcriptional program of PATS/ADI transitional cells that emerge in response to lung injury. We note that in scRNA-seq data, levels of both PATS/ADI and AT1 signatures are not necessarily higher in specific Foxa1/2-deleted clusters compared to controls, but that a higher fraction of cells express these programs, which likely explains their relative enrichment in bulk RNA-seq data.

Global pathway analysis (GSEA) of organoid RNAseq provided additional insights into the cellular identities adopted by FoxA1/2-negative cells. We find consistent enrichment of gene sets canonically expressed in maturing stratified squamous epithelium in FoxA1/2-negative organoids across multiple MSigDB collections. Genes sets associated with keratinocyte differentiation, the cornified envelope, and more broad categorizations of squamous tumors and epidermal cell differentiation are all enriched in FoxA1/2-negative organoids (Figure 4F-G, Supplemental Table 5). GSEA of differentially expressed genes in each cluster (Supplemental Table 3) revealed that similar array of gene sets associated with stratified squamous differentiation were highly enriched in FoxA1/2-negative Cluster 7 (Figure 4H; Supplemental Figure 4E). Specific genes highly expressed in FoxA1/2-negative cells in vivo include Cnfn, a marker of the superficial layers of the stratified squamous epithelium (hereafter SSSE), and Cryab, a reported marker of SSSE and AT1 cells (Figure 4I). IHC for alpha crystallin- $\beta$ chain (the Cryab gene product), revealed expression specifically in a subpopulation of Foxa1/2 deleted tumor cells, but not controls (Figure 4J). Of note, in our analysis this protein was detectable in normal esophageal SSSE but not AT1 cells (Supplemental Figure 4F). Despite induction of several SSSE marker genes, $\Delta$ Np63 is undetectable in FoxA1/2-negative tumor cells (Supplemental Figure $4 \mathrm{G}$ ). $\Delta$ Np63 is a master regulator of squamous differentiation that is normally expressed in the basal layer of stratified squamous epithelia, including the stem cell compartment, and can function as an oncogene in squamous cell carcinoma (SCC) $)^{56}$. Thus, Foxa1/2 deletion causes an incomplete shift toward a squamous differentiation program in a subset of LUAD cells, but the absence of $\Delta \mathrm{Np} 63$ may prevent full squamous transdifferentiation. 
Taken together, these data show that FoxA1/2 inhibit alternative alveolar and stratified squamous differentiation states in NKX2-1-positive LUAD. Specifically, Foxa1/2 deletion causes a partial loss of AT2-like identity in LUAD, with a concomitant increase in the percentage of cells expressing markers of alternative alveolar cell types, such as AT1 or ADI. Moreover, a subset of LUAD cells also de-repress transcriptional programs associated with the SSSE after Foxa1/2 deletion.

\section{NKX2-1 is responsible for a subset of gene expression changes induced by Foxa1/2 deletion}

NKX2-1 has been implicated in the activation of both AT2 and AT1 cell fates and regulates a distinct set of genes

in each cell type $e^{22,23}$. We therefore decided to investigate whether changes in NKX2-1 activity might drive the changes in pulmonary identity programs caused by Foxa1/2 deletion.

We first sought to determine which gene expression changes induced by Foxa1/2 deletion can be attributed to NKX2-1 transcriptional activity. In order to answer this question, we performed RNAseq on organoid cultures transduced with shNKX2-1 or shScramble after treatment with 4-OHT or vehicle ( $\mathrm{n}=2$ biological replicates, Supplemental Figure 5A). Intersection of differentially expressed genes from each of the 4 conditions (Supplemental Figure 5B; Supplemental Table 6) reveals that NKX2-1 is required for $\sim 25 \%$ of gene expression changes caused by Foxa1/2 deletion. Specifically, $22 \%$ of the genes induced by Foxa1/2 deletion (86/396) and $31 \%$ of genes that decline after Foxa1/2 deletion are NKX2-1-dependent (208/663; Figure 5A).

We then investigated how NKX2-1 contributes to the activation of the AT1 and AT2 identities at the program level. Both the AT1 and AT2 transcriptional signatures are enriched in the FoxA1/2-negative/NKX2-1-positive organoids in comparison to the FoxA1/2-negative/NKX2-1-knock-down organoids. This indicates that overall, NKX2-1 is important for both the maintenance of residual AT2 identity and the activation of the de novo AT1 phenotype after Foxa1/2 deletion (Figure 5B). At the individual gene level, we observed nuanced transcriptional dynamics of key AT2 markers. The expression of hallmark surfactant proteins critical for AT2 function, Sftpc and Sftpb, are regulated by both FoxA1/2 and NKX2-1 in an additive manner. Deletion of Foxa1/2 and knock down of NKX2-1 both independently decrease the expression of these genes. A combination of both of these conditions yields the maximal decrease in transcription, illustrating that FoxA1/2 and NKX2-1 can each partially activate 
these AT2 marker genes independent of one another (Figure 5C). Other AT2 marker genes are primarily reliant on the presence of either FoxA1/2 or NKX2-1, but not both. For example, expression of Lamp3 and Sftpa1 is maximally decreased upon Foxa1/2 deletion, and Nkx2-1 knockdown inhibits their expression only in FoxA1/2positive cells, but not FoxA1/2-negative cells, indicating that expression of these genes is primarily dependent on FoxA1/2 (Figure 5D). Conversely, Napsa expression exhibits dependence solely on NKX2-1. Loss of FoxA1/2 does not decrease expression, and NKX2-1 knockdown decreases Napsa transcription to the same extent in both FoxA1/2-positive and FoxA1/2-negative cells (Figure 5E). The complex transcriptional hierarchy between these transcription factors at AT2 marker genes explains why deleting Foxa1/2 decreases, but does not completely ablate, the AT2 signature in NKX2-1-positive LUAD.

In addition to investigating pneumocyte cell fate regulation, we also asked whether NKX2-1 contributes to the activation of the SSSE in the absence of FoxA1/2. We found that SSSE gene sets (Figure 4F, Supplemental Figure 4E) are also enriched in the FoxA1/2-negative/NKX2-1-positive organoids when compared to the FoxA1/2-negative/NKX2-1-knock-down organoids, indicating that NKX2-1 is important for full activation of the SSSE phenotype induced by Foxa1/2 deletion (Figure 5B). At the individual gene level, a subset of SSSE genes are NKX2-1-dependent (e.g. Krt14 and Lor, Figure 5F), whereas others are NKX2-1 independent (e.g. Krt16 and Cldn3, Figure 5G). These data show that NKX2-1 contributes to, but is only partially responsible for, activation of the SSSE identity induced by Foxa1/2 deletion, suggesting that there are other factors that coordinate with NKX2-1 for full expression of the observed SSSE lineage switch.

\section{FoxA1/2 regulate cell type-specific NKX2-1 binding in lung adenocarcinoma}

To further understand the mechanism by which NKX2-1 drives unique transcriptional programs after Foxa1/2 deletion, we performed Chromatin Immunoprecipitation followed by massively parallel DNA sequencing (ChIPseq) for NKX2-1 in FoxA1/2-positive and FoxA1/2-negative organoids ( $n=4$ biological replicates of each condition). We identified a total of 36,958 individual NKX2-1 peaks: 16,911 peaks in FoxA1/2-positive organoids and 32,358 peaks in FoxA1/2-negative organoids via MACS2 (Supplemental Table 7). Initial HOMER transcription factor motif analysis of these two sets of peaks showed that, as expected, NKX binding motifs are enriched in both conditions, whereas FOX motifs are exclusively enriched in FoxA1/2-positive NKX2-1 ChIP 
peaks (Supplemental Figure 6A). Both sets of peaks also share similar genomic occupancy profiles, with a plurality of peaks found in distal intergenic (enhancer) regions, followed by promoters and introns (Supplemental Figure 6B).

Recent publications have shown that in addition to club cells and AT2 cells, NKX2-1 is also expressed in normal AT1 cells, and that differential NKX2-1 localization and transcriptional activity drives alveolar cell fate ${ }^{22,23}$. NKX2-1 is expressed in FoxA1/2-negative tumors (Figure 1A), and FoxA1/2-negative tumors retain residual expression of an AT2 identity while also activating an AT1 program (Figure 4A-E). We therefore investigated whether differential NKX2-1 chromatin binding drives a shift in pulmonary cell identity in the absence of FoxA1/2. Little et $\mathrm{al}^{23}$ performed NKX2-1 ChIPseq in normal mouse AT1 and AT2 cells and categorized all peaks into four groups: AT1-specific binding sites, AT2-specific binding sites, shared general lung epithelial lineage sites, and shared housekeeping sites. In alignment with our gene expression data, there is an overall increase in NKX2-1 localization to its AT1-specific binding sites in the absence of FoxA1/2 (Figure 6A), whereas global NKX2-1 localization at AT2-specific and shared lung epithelial lineage binding sites is unchanged (Figure 6B and Supplemental Figure 6C). NKX2-1 localization at the housekeeping sites also increases upon Foxa1/2 deletion (Supplemental Figure 6D). These data suggest that FoxA1/2 regulates NKX2-1 genomic occupancy, which in turn dictates the specific pulmonary identity adopted by tumor cells.

To investigate differential NKX2-1 genomic localization in more depth, we utilized MAnorm2 ${ }^{57}$ to identify a total of 2,758 high confidence dynamic NKX2-1 binding sites: 1,262 significantly enriched in FoxA1/2-positive cells and 1,496 significantly enriched in FoxA1/2-negative cells (Figure 6C; Supplemental Table 7). When compared to static peaks, a smaller percentage of dynamic peaks are found in promoters, which is accompanied by an increase to $>50 \%$ of peaks localized to distal intergenic (enhancer) regions (Supplemental Figure 6B). HOMER analysis (Supplemental Table 7) of dynamic (Figure 6D-E) and static peaks (Figure 6F) identified two interesting patterns beyond the expected FOX motif and NKX motif distribution. (1) Differential AT2 transcription factor distribution: CEBP-family proteins, NFI-family proteins, GRHL-family proteins, and HNF1 $\beta$ are all transcription factors that play a role in AT2 differentiation ${ }^{23,58-60}$. CEBP motifs are enriched in FoxA1/2-positive and static NKX2-1 peaks, but not in FoxA1/2-negative NKX2-1 peaks. The HNF1 $\beta$ motif is also lost upon Foxa1/2 deletion. 
Instead, we find alternative AT2 transcription factor motifs (NFI and GRHL) in a FoxA1/2-negative context. These data suggest that Foxa1/2 deletion could alter the specific AT2-regulating transcription factors with which NKX2-1 physically interacts and/or binds chromatin. (2) NF $\kappa B$ enrichment in FoxA1/2-negative NKX2-1 ChIP peaks: activation of the NFאB pathway is a hallmark of the ADI/PATS cell population that emerges in response to alveolar injury ${ }^{53,54}$. The presence of an NFאB motif in Foxa1/2 deleted cells raises the possibility of cooperation between NKX2-1 and NFKB signaling in promoting a ADI-like identity.

We also intersected dynamic NKX2-1 peaks with genes that significantly change in response to Foxa1/2 deletion. Overall, we find that direct binding by dynamic NKX2-1 peaks is more significantly associated with gene activation than repression ( $p<0.0001$, Fishers Exact Test; Figure 6G; Supplemental Table 7). Specifically, genes upregulated in FoxA1/2-negative organoids are more strongly associated with NKX2-1 peaks specific to FoxA1/2-negative organoids ( $18 \%$ bound) than with peaks specific to FoxA1/2-positive organoids ( $7 \%$ bound). For example, there are two unique NKX2-1 peaks in Foxa1/2 deleted cells within the promoter region of the AT1 marker Podoplanin (Pdpn; Figure 6H). Similarly, genes upregulated in FoxA1/2-positive organoids are more strongly associated with NKX2-1 peaks specific to FoxA1/2-positive organoids, including a subset of AT2 marker genes. Although at the global level there is no discernable difference in NKX2-1 localization to unique AT2 binding sites (Figure 6B), there are several AT2 marker genes that require FoxA1/2 for NKX2-1 localization. For example, three NKX2-1 peaks near the transcription start site of Lamp3 and two NKX2-1 peaks in the promoter of Sftpa1 are only present in a FoxA1/2-positive context (Figure 6I-J). Corresponding with the loss of NKX2-1 peaks, Foxa1/2 deletion is sufficient for maximal reduction in Lamp3 and Sftpa1 transcript levels (Figure 5D). In contrast, most NKX2-1 peaks at Sftpb and Sftpc can be found in both FoxA1/2-positive and negative organoids (Figure 6K-L). This likely explains why loss of both FoxA1/2 and NKX2-1 is required for full inhibition of their transcription (Figure 5C). Expression of AT2 marker Napsa is not impacted by Foxa1/2 deletion, but does decrease upon NKX2-1 knock down. Correspondingly, NKX2-1 binds within the promoter of Napsa independently of FoxA1/2 (Figure 5E, 6M).

Taken together, these data underscore the complex transcriptional relationship between FoxA1/2 and NKX2-1 in regulation of pulmonary identity. FoxA1/2 regulate cell type-specific localization of NKX2-1 in lung 
adenocarcinoma, and the dynamic NKX2-1 binding sites observed in the context of Foxa1/2 deletion are correlated with differential gene expression leading to the activation of AT1 and AT2 cellular identities.

\section{NKX2-1 impairs tumor growth in the absence of FoxA1/2}

After we established the role of NKX2-1 in regulation of LUAD cellular identity changes following Foxa1/2 deletion, we next wanted to determine whether de novo NKX2-1 transcriptional activity and genomic localization contribute to the growth arrest caused by FoxA1/2 loss. Stochastic NKX2-1 loss can facilitate progression of KP $\operatorname{LUAD}^{17}$. We therefore predicted that if NKX2-1 restrains the growth of Foxa1/2-deleted tumors, there would also be selection for NKX2-1 loss in Foxa1/2-deleted tumors over time. Histopathologic analysis of tamoxifen treated mice in the survival study (Figure 1D) revealed two classes of tumors that grew to macroscopic size despite the absence of FoxA1/2. These macroscopic tumors exhibited morphologies that are highly distinct from the majority of FoxA1/2-negative tumors, which remained microscopic and were indistinguishable from FoxA1/2-negative tumors evaluated two weeks after deletion (Figure 1A-C). The first class, found in $80 \%$ of tamoxifen treated $\mathrm{KPF}_{1} \mathrm{~F}_{2}$ mice ( $\mathrm{n}=8 / 10$ mice histologically evaluated), is characterized by a keratinizing squamous cell carcinoma (SCC) morphology (Supplemental Table 1). These SCCs express squamous lineage specifier $\Delta$ Np63 and retain expression of NKX2-1, albeit at reduced levels compared to adenocarcinomas (Supplemental Figure 7A). The second class, found in $30 \%$ of tamoxifen treated $\mathrm{KPF}_{1} \mathrm{~F}_{2}$ mice $(n=3 / 10)$, consists of poorly differentiated, high grade tumors that are NKX2-1-negative (Figure 7A). In contrast, all poorly-differentiated, NKX2-1-negative tumors observed in vehicle treated $\mathrm{KPF}_{1} \mathrm{~F}_{2}$ mice $(n=9 / 10)$ expressed FoxA1 and/or FoxA2, suggesting that the combined absence of NKX2-1 and FoxA1/2 is specifically found in the context of Foxa1/2 genetic deletion. Since Foxa1/2 deletion does not directly lead to loss of NKX2-1 or gain of $\Delta$ Np63 expression (Figure $1 \mathrm{~A}$ and Supplemental Figure 4E), these observations point to specific stochastic events that might enable LUAD to escape Foxa1/2 deletion over time. Consistent with the first possibility, a stochastic decrease in NKX2-1 levels correlated with sarcomatoid morphology and eventual outgrowth of subcutaneous tumors following Foxa1/2 deletion (Supplemental Figure 2B, right column).

Based on these correlations, we sought to determine whether loss of NKX2-1 is sufficient to rescue Foxa1/2 deletion using multiple independent approaches. First, we initiated Kras ${ }^{G 12 D}$-driven p53-proficient tumors, 
allowed tumors to develop for 6 weeks, and then administered tamoxifen to delete Foxa1 alone $\left(\mathrm{KF}_{1}\right)$, Foxa1 and Foxa2 $\left(\mathrm{KF}_{1} \mathrm{~F}_{2}\right), N k x 2-1$ alone $(\mathrm{KN})$, or $N k x 2-1$, Foxa1 and Foxa2 $\left(\mathrm{KNF}_{1} \mathrm{~F}_{2}\right)$. Tumors progressed for an additional 6 weeks before tissue was collected. Nkx2-1 deletion significantly rescued the effects of Foxa1/2 deletion on tumor growth, resulting in a comparable overall tumor burden in $\mathrm{KNF}_{1} \mathrm{~F}_{2}$ and $\mathrm{KN}$ mice (Figure 7B). As expected ${ }^{15,49}$, tumor burden in $\mathrm{KN}$ was highest overall. We have previously shown that $\mathrm{KNF}_{1} \mathrm{~F}_{2}$ mice develop two distinct tumor types (squamocolumnar junction (SCJ)-like and keratinizing squamous cell carcinoma (SCC), also pictured in Supplemental Figure $7 \mathrm{~B})^{13}$. Both subtypes are more proliferative than both $\mathrm{KF}_{1}$ and $\mathrm{KF}_{1} \mathrm{~F}_{2}$ tumors and therefore contribute to the overall increase in tumor burden of $\mathrm{KNF}_{1} \mathrm{~F}_{2}$ mice (tumor burden of individual subtypes quantified in Supplemental Figure 7C). SCC lesions have a higher BrdU incorporation rate than SCJ-like lesions, consistent with the fact that SCC can form macroscopic tumors whereas SCJ-like lesions remain microscopic in size (Figure $7 C)$.

To determine whether NKX2-1 loss can also rescue Foxa1/2 deletion in a p53-deficient context, we introduced an shRNA against NKX2-1 ${ }^{17}$ to $\mathrm{KPF}_{1} \mathrm{~F}_{2}$ cell lines and organoids in order to decrease NKX2-1 levels alongside deletion of Foxa1/2 via 4-OHT treatment (Figure 7D; Supplemental Figure 5A). Knockdown of NKX2-1 partially rescues the proliferation defect observed upon Foxa1/2 deletion in both 2D cell lines (Figure 7E) and organoid cultures (Figure 7F). As expected from previous work ${ }^{15,17}$, NKX2-1 knockdown also enhances proliferation in parental FoxA1/2-positive cell lines and organoids. In order to determine whether NKX2-1 knockdown prevents tumor regression upon FoxA1/2 loss, we subcutaneously injected NSG mice with the 3311 cell line carrying shNKX2-1 and treated mice with either vehicle or tamoxifen when tumors reached an average volume of $200 \mathrm{~mm}^{3}$. We found that NKX2-1 knockdown increases the rate of growth in both the tamoxifen and vehicle treatments. Importantly, NKX2-1 knock down largely prevents the tumor regression following Foxa1/2 deletion seen in the shScramble tamoxifen treated cohort (Figures 2D and 7G, Supplemental Figure 7D-E). Together, these data demonstrate that aberrant NKX2-1 activity is one mechanism by which FoxA1/2 loss inhibits LUAD growth. 


\section{DISCUSSION}

LUAD progression is driven by epigenetic changes that lead to increased plasticity relative to normal cells of origin, which ultimately enables cancer cells to undergo profound changes in cell identity that enhance malignant potential $^{1-3,5-12}$. Here we show that FoxA1/2 regulate critical aspects of lineage plasticity and infidelity, coordinately driving multiple cell identity programs that are essential for LUAD progression (Figure $7 \mathrm{H}$, top). In the absence of FoxA1/2, LUAD cells undergo lineage switching to alternative identities normally associated with low levels of proliferation (AT1 and SSSE). However, a subset of FoxA1/2-negative LUAD cells stochastically escape this proliferative arrest over time, undergoing an additional lineage switch (to either SCC or poorly differentiated, NKX2-1-negative carcinoma) that enables them to regain their full malignant potential (Figure $7 \mathrm{H}$, bottom).

FoxA1/2 can activate pulmonary and non-pulmonary identities within a single cell in lung adenocarcinoma In NKX2-1-positive, p53-deficient LUAD, FoxA1/2 are required for expression of multiple cellular identity programs, including an AT2-like program and various programs associated with endodermal/GI-like differentiation states. Moreover, we find that FoxA1/2 can activate both AT2 and GI-like differentiation programs within the same cancer cell. This was particularly striking because our previous work in p53-proficient LUAD mouse models showed that FoxA1/2 are restricted to pulmonary activity when NKX2-1 is expressed, and can only activate $\mathrm{Gl}$ programs upon NKX2-1 loss ${ }^{15}$. Specific mechanisms that promote expanded FoxA1/2 transcriptional activity in p53-deficient LUAD are unknown and will require additional investigation. It is likely that a combination of altered histone modification and DNA methylation profiles, as well as changes in cofactor relationships, could enable FoxA1/2 to access non-pulmonary targets in KP LUAD that it cannot bind in p53proficient LUAD (or normal lung epithelium). Moreover, we have recently found that oncogenic signaling can modulate cellular identity in NKX2-1-negative $L U A D^{61}$. We speculate that the increased signaling downstream of KRAS ${ }^{\mathrm{G} 12 \mathrm{D}}$ observed during progression in $\mathrm{KP} \mathrm{LUAD}^{62}$ may also contribute to the capacity of FoxA1/2 to activate GI-related differentiation programs despite the expression of NKX2-1.

FoxA1/2 promote LUAD growth by multiple mechanisms, including regulation of NKX2-1 activity

NKX2-1-positive LUAD growth is highly dependent on FoxA1/2. Deletion of Foxa1/2 in autochthonous lung tumors, murine organoids, and human LUAD cell lines severely impedes proliferation, which in turn significantly 
increases survival. This intense dependence on FoxA1/2 is likely due to a combination of at least two distinct mechanisms, the first being the loss of the dual identity state associated with progression into high grade disease. Additional studies will be needed to determine how co-activation of $\mathrm{Gl}$ and pulmonary programs in NKX2-1positive disease promotes tumor progression. However, we have previously shown that HNF4 $\alpha$, which is a direct FoxA1/2 target in non-pulmonary tissues, promotes the initiation of p53-proficient, NKX2-1-negative LUAD ${ }^{15}$. This raises the possibility that the growth of p53-deficient, NKX2-1-positive LUAD might also be augmented by HNF4 $\alpha$ and other endodermal lineage specifiers, many of which may be direct FoxA1/2 targets outside the lung.

Second, our data suggest that FoxA1/2-dependence in LUAD is also due to novel NKX2-1 activity that, in the absence of FoxA1/2, triggers the activation of transcriptional programs associated with non-proliferative cell types, including AT1 cells and maturing cells of the superficial stratified squamous epithelium. In KP LUAD, FoxA1/2 regulate NKX2-1 localization, resulting in the expression of AT2-specific targets. Upon Foxa1/2 deletion, NKX2-1 exhibits de novo transcriptional activity that contributes to the activation of AT1 and SSSE identities. Under normal conditions, both of these cell types are non-proliferative, and typically require pools of other cell types to differentiate and replace them upon injury or death (AT2/ADI cells and basal epithelial stem cells, respectively). In further support of this hypothesis, concomitant loss of NKX2-1 alongside FoxA1/2 prevents the significant antiproliferative impact of Foxa1/2 deletion alone, as well as the emergence of these non-proliferative cellular identities. Altogether, these data suggest a model in which novel NKX2-1 transcriptional activity after FoxA1/2 loss restrains tumor growth through the activation of alternative, non-proliferative cell identity programs.

\section{FoxA1/2-negative tumors can overcome growth arrest via additional lineage switching}

Despite the severe antiproliferative consequences of Foxa1/2 deletion, we have identified two categories of tumors that stochastically escape this fate over extended periods of time: fully keratinized, NKX2-1 positive SCCs or high grade, poorly differentiated NKX2-1 negative carcinomas. This raises the question of whether all Foxa1/2deleted cells are equally capable of progressing to these two fates, or if only a subset of cells are poised to undergo the stochastic lineage switch required to resume proliferation in a FoxA1/2-negative state. Although our data cannot answer this question directly, we speculate that cells within Cluster 7 may be the most poised to overcome Foxa1/2 deletion-induced growth arrest over time. These cells retain expression of the 'Highly Mixed' 
transcriptional program, which is associated with increased tumorigenic potential in KP LUAD ${ }^{11}$ and might be permissive for dedifferentiation into the NKX2-1 negative, poorly differentiated carcinoma. Furthermore, a subgroup of cells within Cluster 7 have adopted an SSSE-like transcriptional identity. Those cells that co-express SSSE and 'Highly Mixed' programs may be the most likely to give rise to the fully keratinized squamous cell carcinoma escaper tumors in response to stochastic upregulation of $\Delta \mathrm{Np63}$. Technical innovations enabling Foxa1/2 deletion in specific subpopulations of KP LUAD will help to address this question in the future.

Identifying the master transcriptional regulators of cellular identity and investigating the impact of their inactivation is critical for understanding of mechanisms of cancer progression and will contribute to the development of differentiation state-specific therapeutic strategies. Although transcription factors have been historically viewed as undruggable, recent advances in targeted degradation have the potential to make transcription factor inhibition a pharmacologically tractable strategy in cancer ${ }^{63}$. This study suggests that targeting FoxA1/2 may have a major impact on NKX2-1-positive LUAD, including tumors driven by currently undruggable KRAS mutants such as G12D. We also identify candidate mechanisms of escape from FoxA1/2 inhibition, which will likely require co-targeting in order to forestall resistance and achieve a durable response to this potential therapeutic approach.

Acknowledgements: We are grateful to members of the Snyder lab for suggestions and comments. We thank Brian Dalley for sequencing expertise, James Marvin for FACS expertise, Benjamin Spike for scRNAseq analysis expertise, and University of Utah core facilities (PRR, BMP, DNA sequencing, Genomics/Bioinformatics, Flow Cytometry). ELS was supported grants from NIH (R01CA212415 and R01CA240317), a Career Award for Medical Scientists from the Burroughs Wellcome Fund, and institutional funds (Department of Pathology and Huntsman Cancer Institute/Huntsman Cancer Foundation, University of Utah). CRV was supported by Pershing Square Sohn Cancer Research Alliance, the Cold Spring Harbor Laboratory and Northwell Health Affiliation, the National Cancer Institute 5P01CA013106-Project 4 and 1R01CA229699, the Thompson Family Foundation, the Simons Foundation, and a Career Development Award from the Pancreatic Cancer Action Network-American Association for Cancer Research 16-20-25-VAKO. GO was supported by the NIH (F31CA243427) and the Eunice Kennedy Shriver National Institute of Child Health \& Human Development of the National Institutes of 
Health (T32HD007491). OK was supported by the Deutsche Forschungsgemeinschaft (DFG) Research Fellowship (KL 3228/1-1). Research reported in this publication utilized shared resources (including Flow Cytometry, High Throughput Genomics, Bioinformatics, and Biorepository and Molecular Pathology) at the University of Utah and was supported by the National Cancer Institute of the National Institutes of Health under Award Number P30CA042014. Work in the flow cytometry core was also supported by the National Center for Research Resources of the National Institutes of Health under Award Number 1S20RR026802. The content is solely the responsibility of the authors and does not necessarily represent the official views of the NIH.

Competing interests: CRV has received consulting fees from Flare Therapeutics, Roivant Sciences, and C4 Therapeutics, has served on the scientific advisory board of KSQ Therapeutics, Syros Pharmaceuticals, and Treeline Biosciences, has received research funding from Boehringer-Ingelheim and Treeline Biosciences, and owns a stock option from Treeline Biosciences.

Author contributions: GO and ELS designed experiments. GO, AJ, GF, and KG performed experiments. GO, GF, BL, TP, and ELS analyzed data. ELS performed histopathologic review. OK, CRV and KK provided essential tools and reagents. GO and ELS wrote the manuscript. All authors discussed results, reviewed and revised the manuscript. 


\section{Supplemental Tables}

Table 1: Detailed histological analysis of $\mathrm{KP}, \mathrm{KPF}_{1}, \mathrm{KPF}_{2}, \mathrm{KPF}_{1} \mathrm{~F}_{2}$, and $\mathrm{BPF}_{1} \mathrm{~F}_{2}$ survival studies

Table 2: Mouse Cell Atlas analysis of scRNAseq data

Table 3: Differentially expressed genes in $\mathrm{KPF}_{1} \mathrm{~F}_{2}$ organoid isogenic pairs as determined by DEseq2

Table 4: scRNAseq tumor cell barcodes, cluster DEG, and cluster GSEA

Table 5: Significantly enriched gene sets in $\mathrm{KPF}_{1} \mathrm{~F}_{2}$ organoid isogenic pairs as determined by GSEA

Table 6: Differentially expressed genes in $\mathrm{KPF}_{1} \mathrm{~F}_{2}$ shScramble and shNKX2-1 organoid isogenic pairs as determined by DEseq2

Table 7: NKX2-1 ChIPseq (all peaks, differential analysis, gene intersection, HOMER analysis) 


\section{METHODS}

\section{Mice, tumor initiation, tamoxifen administration, and BrdU administration in vivo}

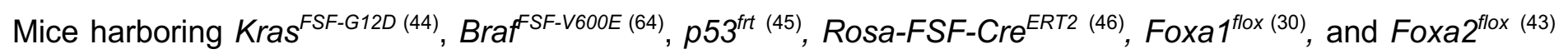
have been previously described. All animals were maintained on a mixed 129/B6 background. Animal studies were approved by the IACUC of the University of Utah, conducted in compliance with the Animal Welfare Act Regulations and other federal statutes relating to animals and experiments involving animals, and adhered to the principles set forth in the Guide for the Care and Use of Laboratory Animals, National Research Council (PHS assurance registration number A-3031-01).

Autochthonous lung tumors were initiated by administering viruses via intratracheal intubation. Adenoviruses were obtained from University of lowa Viral Vector Core. Lentivirus was generated as described in Lentiviral production and transduction methods section.

\begin{tabular}{|l|l|l|}
\hline Experiment & Virus & PFU/mouse \\
\hline Figure 1A-C; Supplemental Figure 1D & Adeno-CMV-FIpO & $4 \times 10^{7}$ \\
\hline Figure 1D-E, 7A; Supplemental Figure 1 F-G, 7A & Lenti-EFS-FlpO & $1 \times 10^{7}$ \\
\hline Supplemental Figure 1E,I & Adeno-mSPC-FlpO & $2.5 \times 10^{8}$ \\
Figures 3-4 & & $5 \times 10^{8}$ \\
Figure 7B-C; Supplemental Figure 7 B-C & & $1 \times 10^{9}$ \\
\hline
\end{tabular}

Tumor-specific activation of Cre $\mathrm{ERT2}^{\mathrm{ER}}$ nuclear activity was achieved by intraperitoneal injection of tamoxifen (Sigma) dissolved in corn oil at a dose of $120 \mathrm{mg} / \mathrm{kg}$. Mice received 4 injections over the course of 5 days. For survival experiments, mice were additionally given pellets supplemented with $500 \mathrm{mg} / \mathrm{kg}$ tamoxifen (Envigo) for 7 days following injections. BrdU incorporation was performed by injecting mice at 40mg/kg (Sigma) intraperitoneally 1 hour prior to tissue collection. Mice in survival studies were monitored for lethargy and respiratory distress, at which time animals were euthanized. 


\section{Analysis of primary human lung adenocarcinoma}

Whole sections of de-identified formalin fixed, paraffin-embedded (FFPE) LUAD $(n=42)$ were obtained from the Intermountain Biorepository, which collects samples in accordance with protocols approved by the Intermountain Healthcare Institutional Review Board. An additional 92 de-identified tumors were evaluated by FFPE tissue microarrays obtained from US Biomax (BC04115c and BCS04017).

\section{Histology and immunohistochemistry}

All tissues were fixed in $10 \%$ formalin overnight and when necessary, lungs were perfused with formalin via the trachea. Organoids were first fixed in $10 \%$ formalin overnight and then mounted in HistoGel (Thermo Fisher Scientific). Mounted organoids and tissues were transferred to $70 \%$ ethanol, embedded in paraffin, and fourmicrometer sections were cut. Immunohistochemistry (IHC) was performed manually on Sequenza slide staining racks (Thermo Fisher Scientific). Sections were treated with Bloxall (Vector Labs) followed by Horse serum 536 (Vector Labs) or Rodent Block M (Biocare Medical), primary antibody, and HRP-polymer-conjugated secondary antibody (anti-Rabbit, Goat and Rat from Vector Labs; anti-Mouse from Biocare. The slides were developed with Impact DAB (Vector Labs) and counterstained with hematoxylin. Slides were stained with antibodies to FoxA1 (1:4000, Abcam 10881-14), FoxA2 (1:1200, Abcam 4466), Murine NKX2-1 (1:2000, Abcam EP1584Y), Human NKX2-1 (1:2000, Abcam 133638), BRDU (1:400, Abcam BU1/75), proSP-B (1:3000, Millipore AB3430), proSPC (1:4000, Millipore AB3786), HNF4 $\alpha$ (1:500, CST C11F12), $\Delta$ Np63 (1:100, Biocare [clone BC28]). Images were taken on a Nikon Eclipse Ni-U microscope with a DS-Ri2 camera and NIS-Elements software. Tumor Burden and BrdU quantitation and histological analyses were performed on hematoxylin and eosin-stained and IHCstained slides using NIS-Elements software. All histopathologic analysis was performed by a board-certified anatomic pathologist (E.L.S.).

\section{MicroCT imaging and analysis}

Mice were anesthetized with isoflurane and imaged using a small animal Quantum GX2 microCT (Perkin Elmer). Images were acquired with 2 minute scans at $90 \mathrm{kV}$ and $88-\mu \mathrm{A}$ current, and reconstructed at a $90-\mu \mathrm{m}$ voxel size. Resulting images were processed with Analyze 12.0 software (Analyze Direct) as described in Mollaoglu et al. $2017^{65}$. 


\section{Establishing primary murine LUAD cell lines and organoids}

Five months after tumor initiation in $\mathrm{KF}_{1} \mathrm{~F}_{2}$ mice (2D cell lines; Lenti-CA2-FlpO-shp53) and $\mathrm{KPF}_{1} \mathrm{~F}_{2}$ mice (3D organoid lines; Adeno-mSPC-FlpO), tumor bearing mice were euthanized and lungs were isolated. Individual macroscopic tumors were removed from lungs, minced under sterile conditions, and digested at $37^{\circ} \mathrm{C}$ for 30 minutes with continuous agitation in a solution of Advanced DMEM/F12 containing the following enzymes: Collagen Type I (Thermo Fisher Scientific, 450U/ml), Dispase (Corning, 5U/ml), DNasel (Sigma, 0.25mg/ml). Enzyme reactions were stopped by addition of cold DMEM/F-12 with $10 \%$ FBS. The digested tissue was repeatedly passed through a 20 -gauge syringe needle, sequentially dispersed through $100 \mu \mathrm{m}, 70 \mu \mathrm{m}$, and $40 \mu \mathrm{m}$ cell strainers, and treated with erythrocyte lysis buffer (eBioscience) to obtain a single cell suspension.

Standard cultures were established by seeding tumor cells in adherent culture flasks. Organoid cultures were established by seeding $1 \times 10^{5}$ tumor cells in 50ul of Matrigel (Corning) and plated in 24-well plates. For the first 1-2 weeks of organoid initiation, Matrigel droplets were overlaid with recombinant organoid medium: Advanced DMEM/F-12 supplemented with 1X B27 (Gibco), 1X N2 (Gibco), 1.25mM nAcetylcysteine (Sigma), 10mM Nicotinamide (Sigma), 10nM Gastrin (Sigma), 100ng/ml EGF (Peprotech), 100ng/ml R-spondin1 (Peprotech), 100ng/ml Noggin (Peprotech), and 100ng/ml FGF10 (Peprotech). After organoids were established, cultures were switched to $50 \%$ L-WRN conditioned media ${ }^{66}$.

Organoid cultures were screened via immunohistochemistry and qPCR, and lines that uniformly expressed FoxA1, FoxA2, and NKX2-1 in all cells were selected for subsequent analysis. Standard culture cell lines were heterogeneous for NKX2-1, with both NKX2-1-positive and NKX2-1-negative cells identified within the same parental culture. In order to generate uniform cultures for downstream analysis our 2D cell lines were single cell cloned. The clonal populations were screened via immunoblotting for FoxA1, FoxA2, and NKX2-1, and the cell line with strongest expression of all three transcription factors was selected for subsequent analysis. The full name of the 2D cell line in this paper labeled 3311 is 3311-Tumor 3-Subclone 1, which was shortened for clarity.

\section{Cell culture}

Standard culture murine cell lines and HEK293T cells were cultured in DMEM/10\% FBS (Gibco). Murine 
organoids were cultured within Matrigel (Corning or Preclinical Research Shared Resource core facility) submerged in 50\% L-WRN conditioned media. All cell lines were tested periodically for mycoplasma contamination. To maintain cell cultures mycoplasma free, all culture media were supplemented with $2.5 \mathrm{ug} / \mathrm{ml}$ Plasmocin. Cells were transiently treated with $2 \mu \mathrm{M}$ 4-hydroxytamoxifen (Cayman Chemical Company, dissolved in $100 \%$ Ethanol) or vehicle for 48 (standard culture) or 72 (organoid culture) hours to activate CreER $^{\mathrm{T} 2}$ nuclear activity and generate isogenic pairs.

\section{Immunoblotting}

Cells were lysed on ice for 20 minutes in RIPA buffer (50mM Tris $\mathrm{HCl} \mathrm{pH} \mathrm{7.4,} 150 \mathrm{mM} \mathrm{NaCl}, 0.1 \%$ (w/v) SDS, $0.5 \%(\mathrm{w} / \mathrm{v})$ sodium deoxycholate, $1 \%(\mathrm{v} / \mathrm{v})$ Triton X-100) plus complete protease phosphatase inhibitor cocktail (A32961, Thermo Fisher Scientific). Cellular debris was pelleted for 15 minutes at $4{ }^{\circ} \mathrm{C}$ and protein concentration was quantitated with the Coomassie (Bradford) Protein Assay (ThermoFisher Scientific). A total of $15 \mu \mathrm{g}$ (organoids) or $30 \mu \mathrm{g}$ (cell lines) of protein lysates were separated on Tris-Glycine (TGX) precast gels (BIO RAD) and transferred to nitrocellulose membranes (Thermo Fisher Scientific). Membranes were probed overnight with antibodies to FoxA1 (1:1000, Abcam 23738), FoxA2 (1:1000, Abcam 108422), NKX2-1 (1:2000, Abcam 133638), and Vinculin (1:20000, Abcam 129002). The next day membranes were probed with IRDye 800CW Goat antiRabbit IgG Secondary Antibody (1:20000, LI-COR) and imaged with a LI-COR Odyssey CLx and Image Studio Software.

\section{Generating a single cell suspension from organoid cultures}

Matrigel droplets containing organoid cells were broken down via repeated pipetting in Cell Recovery Solution (Corning, $500 \mu \mathrm{l}$ per Matrigel droplet). Cell Recovery Solution containing organoids was transferred to sterile conical tubes and submerged in ice for $20-30$ minutes before centrifugation at $4^{\circ} \mathrm{C}(300-500 \mathrm{G})$. Cell Recovery Solution supernatant was removed and the cell pellet was washed via resuspension in PBS followed by centrifugation. Cells were then resuspended in pre-warmed TrypLE Express Enzyme (Thermo Fisher Scientific) and incubated for 5-7 minutes at $37^{\circ} \mathrm{C}$. TrypLE reaction was quenched via dilution with cold Splitting Media (Advanced DMEM/F-12 [Gibco], 10 mM HEPES [Invitrogen], 1X Penicillin-Streptomycin-Glutamine [Invitrogen]). Cells were centrifuged and then resuspended in a pre-warmed DNase solution (L-WRN media supplemented to 
a final concentration of $200 \mathrm{U} / \mathrm{ml}$ DNase [Worthington], $2.5 \mathrm{mM} \mathrm{MgCl}, 500 \mu \mathrm{M} \mathrm{CaCl}_{2}$ ) and incubated for 5-7 minutes at $37^{\circ} \mathrm{C}$. Cells were centrifuged and washed in PBS before use.

\section{Cell viability and growth assays}

EDU Incorporation Assay: One week after 4-OHT/Ethanol treatment, Matrigel droplets containing isogenic organoid cells were incubated in L-WRN/100mM EdU for 30 minutes. EdU solution was then removed and droplets were washed with $500 \mu$ l of warm PBS before generating a single cell suspension. The single cell suspension was fixed in 1ml of PFA in PME Buffer for 20 minutes (4\% Formaldehyde [Pierce], 500 mM PIPES, $25 \mathrm{mM} \mathrm{MgCl}_{2}, 50 \mathrm{mM}$ EDTA in UltraPure Water [Invitrogen]), washed in 1ml of IF Buffer (PBS pH 7.4, 0.20\% Triton X-100, 0.05\% Tween-20), permeabilized for 20 minutes in 1X Click-iT Permeabilization and Wash Reagent (Thermo Fisher), washed in PBS/3\% BSA, incubated shielded from light for 30 minutes in $500 \mu$ of Click-iT Reaction Cocktail (Thermo Fisher), washed in 3ml of 1X Click-iT Permeabilization and Wash Reagent, and resuspended in $500 \mu$ l of DAPI Solution $(1 \mu \mathrm{g} / \mathrm{ml}$ in 1 X Click-iT Permeabilization and Wash Reagent). Cells were analyzed on a BD LSR Fortessa. Cells were first passed through routine light-scatter and doublet discrimination gates before being concurrently analyzed for DAPI signal for cell cycle distribution (BV421, Area) vs EdU signal for actively replicating cells (Alexa Fluor 647, Area).

Incucyte Live-Cell Imaging: Standard culture cells were seeded at a density of 7,000 cells per well $(3311), 10,000$ cells per well (H2009), or 20,000 cells per well (H358 and H441) in a 96-well plate and grown within an IncuCyte Live Cell Imaging System. Wells were imaged every two hours. Percent phase confluence was determined using IncuCyte Zoom software. Culture media was changed as needed and the assay was ended once one of the conditions reached $100 \%$ confluency.

CellTiter-Glo Assay: Organoid cells were broken down into a single cell suspension. Four identical 48-well plates were seeded with three wells per condition (5000 cells/well in 15 ul of Matrigel). The following protocol was performed on the day of seeding, and every other day for 6 days following seeding. CellTiter-Glo 3D reagent (Promega) and 50\% L-WRN media were warmed to room temperature. A working solution was prepared with a ratio of 1 part CTG:5 parts L-WRN. Media was removed from each well, $330 \mu$ of CTG working solution was 
added to each well containing organoids and one empty well before shielding from light and incubating at room temperature for 30 minutes on a plate shaker at 500 RPM. After incubation CTG working solution was transferred to a clear-bottom, solid-wall 96 well plate (100ul/well; 3 wells/replicate; 3 replicates/sample). Luminescence was measured using an EnVision 2105 Multimode Plate Reader (Perkin Elmer). Luminescent signal for each condition was normalized to the corresponding Day 0 luminescence read.

\section{Subcutaneous allografts}

For subcutaneous allograft experiments, a single cell suspension of $3 \times 10^{5}$ standard culture cells were mixed in a 1:1 volume with $50 \mu$ of Matrigel. Cells were subcutaneously injected into the flank of NOD/SCID-gamma chain deficient mice (NSG). Tumor dimensions were measured with calipers, and tumor volume was calculated using the $\left(\mathrm{L} \times \mathrm{W}^{2}\right) / 2$ formula. When the average volume of the tumors surpassed $200 \mathrm{~mm}^{3}$, mice were randomized into Corn Oil or Tamoxifen cohorts (120mg/kg Tamoxifen). Mice received daily injections for 4 days. Tumor volume was monitored and measured every 3 days, and mice were euthanized once one tumor within the cohort surpassed $1000 \mathrm{~mm}^{3}$ (Figure 1) or individually as their tumor volume surpassed $1000 \mathrm{~mm}^{3}$ (Figure $4 / 4 \mathrm{~S}$ ).

\section{Lentiviral production and transduction}

Cloning of EFS-FIpO lentivector: We generated a pCDH-EFS-FIpO lentiviral vector by PCR amplifying the EFS (EF1a) promoter from the $\mathrm{pCDH}-\mathrm{Cre}$ plasmid ${ }^{67}$, digesting the purified PCR product with $\mathrm{Xbal}$, and cloning into SnaBI-Xbal sites of $\mathrm{pCDH}-\mathrm{CMV}-\mathrm{FIpO}^{13}$ via ligation with T4 DNA ligase. Correct identity and orientation of the construct was confirmed via Sanger sequencing.

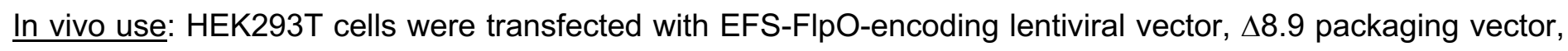
and VSV-G envelope vector mixed with TransIT-293 (Mirus). Virus-containing supernatant was collected 36, 48, 60 , and 72 hours after transfection, and then ultracentrifuged at 25,000 RPM for 2 hours to concentrate virus for in vivo infection. The concentration of the final viral stock was determined using the mouse fibroblast FlpO-GFP reporter cell line 3TZ to determine Plaque Forming Units/ $\mu \mathrm{l}$ (PFU).

Cloning of human dual sgRNA constructs: Dual targeting vectors were generated by inserting a sgRNA1scaffold-bovineU6-sgRNA2 cassette (synthesized as gene-blocks by IDT) into the LRG2.1-GFP-P2A-BlastR 
vector using Gibson assembly. The scaffold sequence for sgRNA was generated by altering the stem-stem loop region of the LRG2.1 scaffold $^{68}$ based on previously described CRISPRi sgRNA scaffolds ${ }^{69}$.

\begin{tabular}{|l|l|l|}
\hline Construct & sgRNA \#1 & sgRNA \#2 \\
\hline sgFoxA\#1 & ATGTACGAGTAGGGCGGCT & TCGCCCGACAAGCCCGGCAA \\
\hline sgFoxA\#10 & CCGTTCTCGAACATGTTGC & AGTCGTTGAAGGAGAGCGAG \\
\hline sgFoxA\#11 & TGGACGCTGCACCCGGACTC & GCTCAGCGTCAGCATCTTGT \\
\hline sgNT & CCGGCGCCGAGCCGGACTTC & TTGCGGTCAGGTCACGCCGC \\
\hline
\end{tabular}

In vitro use: HEK293T cells were transfected with lentiCRISPRv2 (Addgene Plasmid \#98290), the above dual sgRNA constructs, pLKO.shNkx2-1 (Addgene Plasmid \#32400) or pLKO.shScramble (Addgene Plasmid \#1864) lentiviral vectors, $\Delta 8.9$ packaging vector, and VSV-G envelope vector mixed with TransIT-293 (Mirus). Viruscontaining supernatant was collected 48,60 , and 72 hours after transfection, centrifuged to pellet floating HEK293T cells, and filtered using $0.45 \mu \mathrm{m}$ filters before storing long term at $-80^{\circ} \mathrm{C}$.

Human CRISPR/Cas9: Human cell lines were transduced with lentiCRISPRv2 by culturing with undiluted lentiviral media containing $8 \mu \mathrm{g} / \mathrm{ml}$ polybrene for 48 hours total, refreshing the media and polybrene at 24 hours. Three days after transduction ceased, cells were subjected to Puromycin selection to produce stable cell lines expressing Cas9. Cells were then transduced with lentiviral sgNT, sgFoxA\#1, sgFoxA\#10, or sgFoxA\#11 and selected with Blasticidin in the same manner. After three days of selection, cells were seeded for an Incucyte proliferation assay, protein was collected for immunoblotting, and RNA was collected for qRT-PCR analysis.

shRNA: Standard culture cell lines were transduced by culturing with undiluted lentiviral media containing $8 \mu \mathrm{g} / \mathrm{ml}$ polybrene for 48 hours total, refreshing the media and polybrene at 24 hours. Three days after transduction ceased, cells were subjected to Puromycin selection to produce stable lines. For stable transduction of isogenic organoid pairs post-4OHT/Ethanol treatment, Matrigel domes containing organoids were resuspended in ice cold Splitting Media and centrifuged at $300 \mathrm{G} 4^{\circ} \mathrm{C}$ twice. Cells were then resuspended in TrypLE Express Enzyme (Thermo Fisher Scientific), incubated for 5 minutes at $37^{\circ} \mathrm{C}$, and quenched with ice cold Splitting Media. Cell 
pellets were resuspended in a 1:1 mixture, by volume, of $50 \%$ L-WRN and lentiviral media with a final concentration of $8 \mu \mathrm{g} / \mathrm{ml}$ polybrene, 1X Y-27632 (ROCK inhibitor), 1X A83-01 (ALK inhibitor), and 1X SB431542 (ALK inhibitor). The cell suspension was then transferred to a 6 -well plate and centrifuged at 1700 RPM for 1 hour at room temperature. After centrifugation, the plate was incubated at $37^{\circ} \mathrm{C}$ for 2 hours. Cells were collected (scraping the bottom of the plate when necessary), centrifuged, resuspended in Matrigel, seeded in 24-well plates, and cultured in 50\% L-WRN supplemented with 1XY-27632, 1X A83-01, and 1X SB431542 for 72 hours. Three days after transduction, inhibitor media was washed away and cells were subjected to Puromycin selection to produce stable lines.

\section{RNA Sequencing}

RNA was collected from biological replicates of isogenic organoid cultures 1027B, 1027D, and 1292B 3 weeks after 4-OHT/Ethanol treatment. RNA was isolated via Trizol-chloroform extraction followed by column-based purification. The aqueous phase was brought to a final concentration of $50 \%$ ethanol, and RNA was purified using the PureLink RNA Mini kit according to the manufacturer's instructions (ThermoFisher Scientific). Library preparation was performed using the Illumina TruSeq Stranded mRNA Library Prep with UDI (Illumina; poly(A) selection). Sequencing was performed using the NovaSeq 6000 (50 x 50 bp paired-end sequencing; 25 million reads per sample).

\section{RNAseq Data Processing and Analysis}

Mouse FASTA and GTF files were downloaded from Ensembl release 98 and a reference database was created using STAR version 2.7.2 $\mathrm{c}^{70}$ with splice junctions optimized for 50 base pair reads. Optical duplicates were removed from NovaSeq runs via Clumpify v38.34 ${ }^{71}$. Reads were trimmed of adapters and aligned to the reference database using STAR in two pass mode to output BAM file sorted by coordinates. Mapped reads were assigned to annotated genes in the GTF file using featureCounts version 1.6. $3^{72}$. Non-coding features, histones, and ribosomal RNAs were removed and differentially expressed genes were identified using a $5 \%$ false discovery rate with DESeq2 version $1.30 .1^{73}$. 
GSEA-Preranked was run with the differential gene list generated from DESeq2 and the following MSigDB gene sets: c2, c3, c4, c5, c6, c8, and Hallmarks on November 12, 2020. Gene sets smaller than 15 and larger than 500 were excluded from analysis. Marjanovic et al Clusters and Program gene lists and ADI/PATS signatures were downloaded from their supplemental materials.

\section{Evaluation of NKX2-1 dependence}

We first performed differential gene expression analysis (DESeq2) between the following pairs of samples: (1) shScramble vehicle treated (FoxA1/2-positive; NKX2-1-positive) vs shScramble tamoxifen treated (FoxA1/2negative; NKX2-1-positive) (2) shScramble vehicle treated (FoxA1/2-positive; NKX2-1-positive) vs shNKX201 tamoxifen treated (FoxA1/2-negative; NKX2-1-knock down). We then intersected the differential gene lists for these two comparisons. Gene expression changes found in both comparisons were deemed NKX2-1 independent because the expression change is induced upon Foxa1/2 deletion regardless of NKX2-1 levels. Gene expression changes unique to comparison (1) were deemed NKX2-1 dependent because the expression change induced upon Foxa1/2 deletion only occurs when NKX2-1 is fully expressed.

\section{Single Cell RNA Sequencing}

Mice were injected with tamoxifen 10 weeks after intubation and single cells were collected 12 weeks after intubation as follows. Lungs and heart were perfused with PBS. Individual macroscopic tumors were removed from the lungs and broken down into single cell suspensions as described in Establishing primary murine LUAD cell lines and organoids methods. Pre-depletion cells were viably cryopreserved in 5\% DMSO/FBS. The remaining cells were depleted of CD45-positive and CD31-positive cells using MACS with Miltenyi microbeads (CD45: 130-052-301; CD31: 130-097-418) and LD columns (130-042-901) following manufacturer recommendations. Post-depletion cells were viably cryopreserved in 5\% DMSO/FBS.

Protocols used to generate scRNA-seq data with 10x Genomics Chromium platform can be found at https://support.10xgenomics.com/single-cell-gene-expression.

In brief, the Chromium Single Cell Gene Expression Solution with 3' chemistry, version 3 (PN-1000075) was used to barcode individual cells with 16bp 10X barcodes and to tag cell specific transcript molecules with 10bp 
Unique Molecular Identifier (UMI) according to the manufacturer's instructions. The following protocol was performed at the High-Throughput Genomics Shared Resource at Huntsman Cancer Institute, University of Utah. Single cells were suspended in phosphate buffered saline with $0.04 \%$ bovine serum albumin, and the cell suspension was passed through a 40 micron cell strainer. Viability and cell count were assessed on Countess II (Thermo Scientific). Suspensions were equilibrated to targeted cell recovery of 8000 cells. For the KP sample without stromal depletion, the targeted cell recovery was 7500 cells. 10x Gel Beads and reverse transcription reagents were added and cell suspensions were loaded to Chromium Single Cell A (PN-120236) to form Gel Beads-in emulsions (GEMs) - the nano-droplets. Within individual GEMs, cDNA generated from captured and barcoded mRNA was synthesized by reverse transcription at the setting of $53^{\circ} \mathrm{C}$ for 45 min followed by $85^{\circ} \mathrm{C}$ for 5 min. Subsequent A tailing, end repair, adaptor ligation and sample indexing were performed in bulk according to the manufacturer's instructions. The resulting barcoding libraries were qualified on Agilent D1000 ScreenTape on Agilent Technology 2200 TapeStation system and quantified by quantification PCR using KAPA Biosystems Library Quantification Kit for Illumine Platforms (KK4842). Multiple libraries were then normalized and sequenced on NovaSeq 6000 with $2 \times 150$ PE mode.

\section{scRNAseq Data Processing and Analysis}

\section{Demultiplexing and data alignment}

Single-cell RNA-seq data from both $\mathrm{KP}(\mathrm{n}=2)$ and $\mathrm{KPF}_{1} \mathrm{~F}_{2}(\mathrm{n}=2)$ tumors were demultiplexed using the $10 \mathrm{x}$ cellranger mkfastq version 3.1 .0 to create fastq files with the 11 sample index, R1 cell barcode+UMI, and R2 sequence. Reads were aligned to the mouse genome (mm10 with custom CRE-ERT2 and FoxA1/2 individual exon references) and UMls were generated using cellranger count 3.1 .0 with expected-cells set to 8000 per library. For the KP sample without stromal depletion, expected-cells was set to 7500. QC reporting, clustering, and dimension reduction were performed for initial data evaluation in 10x Genomics' Cell Loupe Browser (v5.0). For the KP sample without stromal depletion, we captured 5,963 cells total with 48,090 mean reads per cell and 1,488 median genes per cell. For the KP sample with stromal depletion, we captured 8,536 cells total with 25,593 mean reads per cell and 736 median genes per cell. For the $\mathrm{KPF}_{1} \mathrm{~F}_{2}$ sample without stromal depletion, we captured 4,662 cells total with 55,589 mean reads per cell and 1,552 median genes per cell. For the $\mathrm{KPF}_{1} \mathrm{~F}_{2}$ sample with stromal depletion, we captured 4,415 cells total with 60,226 mean reads per cell and 1,339 median 
genes per cell. Additional details of the primary Cell Ranger data processing can be found at: https://support.10xgenomics.com/single-cell-gene-expression/software/pipelines/latest/algorithms/overview.

\section{Quality control, clustering, and cell type identification}

Single cell expression data was subjected to common Seurat workflows for initial quality control and clustering (https://satijalab.org/seurat/articles/pbmc3k_tutorial.html). Cells with unique feature counts over 7500 or less than 200 and over $20 \%$ mitochondrial counts were filtered out for downstream analysis. Counts of cells passing QC were then log normalized and scaled based on all genes using Seurat's NormalizeData and ScaleData functions. PCA linear dimension reduction was performed and Seurat's FindNeighbors function was employed to embed single cell profiles in a K-nearest neighbor (KNN) graph based on a (30 PC) PCA space. The FindClusters function was utilized to iteratively group cells together using Louvian algorithm modularity optimization techniques. Clustering was performed based on the top 30 dimensions using Seurat's RunUMAP function. Differentially expressed genes for each cluster were identified using Seurat's FindMarkers function using default setting. The Mouse Cell Atlas' scMCA R package was run with the top differentially expressed genes for each cluster (Supplemental Table 2) to facilitate cell type identification. Following QC filtering and tumor cell identification, $4646 \mathrm{KP}$ and $2481 \mathrm{KPF} 1 \mathrm{~F} 2$ high quality tumor cells remained. For subsequent analyses, identified tumor cells from KP and KPF1F2 tumors were subsetted out and reclustered based on the top 25 dimensions. Cell barcodes identified as tumor cells that were used for downstream analyses are included in Supplemental Table 3. Reclustering of identified tumor cells in UMAP space revealed 13 clusters (Figure 3A) To identify $\mathrm{KPF}_{1} \mathrm{~F}_{2}$ complete recombinants, expression of the floxed second exon of Foxa1 was visualized (Figure 3C).

\section{Differential gene expression and signature score assignment}

Differentially expressed genes in each of the tumor cell clusters were calculated using Seurat's FindMarkers function. Differentially expressed genes of UMAP clusters from all KP and $\mathrm{KPF}_{1} \mathrm{~F}_{2}$ tumor cells can be found in Supplemental Table 3. Gene module scores for several published gene signatures for AT1 cells AT2 cells $^{11,22,51,52}$, and various additional cell types and states including stratified squamous epithelium (MSigDB) 
were determined per cell across the tumor cell and complete recombinant data sets using Seurat's AddModuleScore function. Gene lists for each of these scores can be found in Supplemental Table 5.

\section{Chromatin Immunoprecipitation Sequencing}

Organoids were broken down into a single cell suspension before following the manufacturer protocol v1.5 for Chromatrap ChIP-seq Protein A kits (500189) with the following modifications/optimizations:

Step 1 (Chromatin preparation, fixation, collection): Broken down, individual organoid cells were treated as suspension cells for crosslinking.

Step 2 (Cell lysis and chromatin shearing): Crosslinked cells were suspended in 100ul of Lysis Buffer/2,000,000 cells. Sonication was performed with 20 cycles of 30 seconds on:30 seconds off at $70 \%$ to reach fragmentation of $100-500$ bp lengths. Input DNA was purified with Zymo ChIP DNA Clean and Concentrator columns (D5205). DNA concentration was determined using Qubit dsDNA High Sensitivity Assay Kit (Q32851).

Step 3 (Slurry preparation and immunoprecipitation): NKX2-1 IP was performed with a sample:antibody ratio of 10ug sample:4.2ug antibody (Abcam ab133737).

Step 4 (Reverse cross-linking): IP DNA was purified with Zymo ChIP DNA Clean and Concentrator columns (D5205).

IP and input samples were prepared for sequencing with NEBNext Ultra II DNA Library Prep Kit and sequenced on an Illumina Novaseq 6000 as paired-end 150 bp reads (HCI High-Throughput Genomics core).

\section{ChIPseq Data Processing and Analysis}

Samples were de-multiplexed with Illumina BCL2Fastq using dual indexes and single bp mismatch. Fastq reads were aligned to the standard chromosomes of mouse genome, version mm10, using Novocraft novoalign (v4.03.01 http://www.novocraft.com) with provided tuned settings for Novaseq. Sequence adapter sequences were provided to Novoalign for masking during alignment. Alignment pairings were checked with samtools (v1.10) fixmate function. 
Samples were run through the MultiRepMacsChIPSeq pipeline

https://github.com/HuntsmanCancerInstitute/MultiRepMacsChIPSeq) with five independent samples. After comparison, four replicates were chosen for analysis, based on correlation metrics and number of peaks identified. Briefly, the pipeline consists of the following steps. Exclusion intervals representing high-copy genomic sequences were first generated by calling peaks on combined, unfiltered Input samples. Any alignments overlapping exclusion intervals were discarded from further analysis. Only properly-paired alignments were used; chimeric and singleton alignments were discarded. Duplicate alignments based on coordinates were randomly subsampled to a final rate of $5 \%$ across all samples from a mean of $24 \%$ (range $18-32 \%$ ). Peaks were called individually for each replicate using MACS2 (v2.2.6 https://github.com/macs3-project/MACS) with peak calling parameters of qvalue 0.01, min-length $200 \mathrm{bp}$, max-gap $100 \mathrm{bp}$, and genome size (empirically determined) 2554209000 bp. Called peaks were first combined between replicates for each condition, EtOH and Tamoxifen treated, and then combined into a master peak list for both conditions using BEDtools (v2.28.0 https://bedtools.readthedocs.io). Mean fragment coverage tracks were generated using bam2wig (BioToolBox, v1.68 https://github.com/tjparnell/biotoolbox) by first depth-normalizing to 1 million paired-end fragments, then averaging between replicates. Multi-mapping alignments were scaled by the number of genomic hits reported by the aligner. Mean Log2 Fold Enrichment tracks were generated by using MACS2 bdgcmp function with depthnormalized ChIP coverage and generated mean lambda control coverage tracks, then converting to base 2 log values with manipulate_wig (BioToolBox). Count tracks were generated with bam2wig scaling to a total depth of 20M fragments, the lowest observed depth amongst all samples.

Peaks were scored using get_datasets (BioToolBox) with the depth-normalized count files generated by the MultiRepMacsChIPSeq pipeline. Fractional fragment counts were rounded to the nearest integer for simplicity. Differential peaks were identified by running MAnorm $2^{57}$ with recommended parameters, using "parametric" for fitting the mean variance curve and initial coefficients of 0.1 and 10 . Differential peaks were identified by a padj value $<0.05$. Data was collected for peaks by using BED files of the peaks of interest using the BioToolBox (v1.68) programs get_relative_data, using 50 bins of $40 \mathrm{bp}$ surrounding the peak midpoint $( \pm 1 \mathrm{~kb})$. Heat maps and plots were generated with custom R scripts using pHeatmap (https://cran.r-project.org/package=pheatmap) and ggplot2 (https://ggplot2.tidyverse.org). Peaks were annotated with ChIPseeker ${ }^{74}$ and Ensembl gene 
bioRxiv preprint doi: https://doi.org/10.1101/2021.06.29.450247; this version posted November 10, 2021. The copyright holder for this preprint (which was not certified by peer review) is the author/funder. All rights reserved. No reuse allowed without permission.

annotation (release 98), restricted to expressed, protein-coding, Gencode transcripts. Motifs, both known and novel, were identified using the Homer package (http://homer.ucsd.edu/homer/).

\section{Data Availability}

All sequencing data generated in this study are available at Gene Expression Omnibus (GEO accession number pending) 


\section{REFERENCES}

1 Boumahdi, S. \& de Sauvage, F. J. The great escape: tumour cell plasticity in resistance to targeted therapy. Nat Rev Drug Discov 19, 39-56, doi:10.1038/s41573-019-0044-1 (2020).

2 Le Magnen, C., Shen, M. M. \& Abate-Shen, C. Lineage Plasticity in Cancer Progression and Treatment. Annu Rev Cancer Biol 2, 271-289, doi:10.1146/annurev-cancerbio-030617-050224 (2018).

3 Quintanal-Villalonga, A. et al. Lineage plasticity in cancer: a shared pathway of therapeutic resistance. Nat Rev Clin Oncol 17, 360-371, doi:10.1038/s41571-020-0340-z (2020).

4 Meza, R., Meernik, C., Jeon, J. \& Cote, M. L. Lung cancer incidence trends by gender, race and histology in the United States, 1973-2010. PLoS One 10, e0121323, doi:10.1371/journal.pone.0121323 (2015).

5 Travis, W. D. et al. International association for the study of lung cancer/american thoracic society/european respiratory society international multidisciplinary classification of lung adenocarcinoma. J Thorac Oncol 6, 244-285, doi:10.1097/JTO.0b013e318206a221 (2011). Campos-Parra, A. D. et al. Relevance of the novel IASLC/ATS/ERS classification of lung adenocarcinoma in advanced disease. Eur Respir J 43, 1439-1447, doi:10.1183/09031936.00138813 (2014). Niederst, M. J. et al. RB loss in resistant EGFR mutant lung adenocarcinomas that transform to small-cell lung cancer. Nat Commun 6, 6377, doi:10.1038/ncomms7377 (2015). Russell, P. A. W., Z.; Wright, G.M.; Daniels, M.; Conron, M.; Williams, R.A. Does lung adenocarcinoma subtype predict patient survival?: A clinicopathologic study based on the new International Association for the Study of Lung Cnacer/American Thoracic Society/European Respiratory Society international multidisciplinary lung adenocarcinoma classification. Journal of thoracic oncology: official publication of the International Association for the Study of Lung Cnacer, 1496-1504 (2011).

9 Sun, Z. \& Yang, P. Gene expression profiling on lung cancer outcome prediction: present clinical value and future premise. Cancer Epidemiol Biomarkers Prev 15, 2063-2068, doi:10.1158/1055-9965.EPI-06-0505 (2006).

10 Tavernari, D. et al. Nongenetic Evolution Drives Lung Adenocarcinoma Spatial Heterogeneity and Progression. Cancer Discov 11, 1490-1507, doi:10.1158/2159-8290.CD-20-1274 (2021).

11 Marjanovic, N. D. et al. Emergence of a High-Plasticity Cell State during Lung Cancer Evolution. Cancer Cell 38, 229-246 e213, doi:10.1016/j.ccell.2020.06.012 (2020).

12 LaFave, L. M. et al. Epigenomic State Transitions Characterize Tumor Progression in Mouse Lung Adenocarcinoma. Cancer Cell 38, 212-228 e213, doi:10.1016/j.ccell.2020.06.006 (2020).

13 Camolotto, S. A. et al. FoxA1 and FoxA2 drive gastric differentiation and suppress squamous identity in NKX2-1-negative lung cancer. Elife 7, doi:10.7554/eLife.38579 (2018). $\mathrm{Li}, \mathrm{C}$. M. et al. Foxa2 and Cdx2 cooperate with Nkx2-1 to inhibit lung adenocarcinoma metastasis. Genes Dev 29, 1850-1862, doi:10.1101/gad.267393.115 (2015).

15 Snyder, E. L. et al. Nkx2-1 represses a latent gastric differentiation program in lung adenocarcinoma. Molecular cell 50, 185-199, doi:10.1016/j.molcel.2013.02.018 (2013). Watanabe, H. et al. Integrated cistromic and expression analysis of amplified NKX2-1 in lung adenocarcinoma identifies LMO3 as a functional transcriptional target. Genes Dev 27, 197210, doi:10.1101/gad.203208.112 (2013).

17 Winslow, M. M. et al. Suppression of lung adenocarcinoma progression by Nkx2-1. Nature 473, 101-104, doi:10.1038/nature09881 (2011).

Bohinski, R. J., Di Lauro, R. \& Whitsett, J. A. The lung-specific surfactant protein B gene promoter is a target for thyroid transcription factor 1 and hepatocyte nuclear factor 3 , indicating common factors for organ-specific gene expression along the foregut axis. $\mathrm{Mol} \mathrm{Cell} \mathrm{Biol} \mathrm{14,}$ 5671-5681 (1994). 
19 Liu, C., Glasser, S. W., Wan, H. \& Whitsett, J. A. GATA-6 and thyroid transcription factor-1 directly interact and regulate surfactant protein-C gene expression. J Biol Chem 277, 45194525, doi:10.1074/jbc.M107585200 (2002).

20 Yi, M., Tong, G. X., Murry, B. \& Mendelson, C. R. Role of CBP/p300 and SRC-1 in transcriptional regulation of the pulmonary surfactant protein-A (SP-A) gene by thyroid transcription factor-1 (TTF-1). J Biol Chem 277, 2997-3005, doi:10.1074/jbc.M109793200 (2002).

21 Bingle, C. D. Thyroid transcription factor-1. Int J Biochem Cell Biol 29, 1471-1473 (1997).

22 Little, D. R. et al. Transcriptional control of lung alveolar type 1 cell development and maintenance by NK homeobox 2-1. Proc Natl Acad Sci U S A 116, 20545-20555, doi:10.1073/pnas.1906663116 (2019).

23 Little, D. R. et al. Differential chromatin binding of the lung lineage transcription factor NKX2-1 resolves opposing murine alveolar cell fates in vivo. Nat Commun 12, 2509, doi:10.1038/s41467-021-22817-6 (2021).

24 Minoo, P. et al. Physical and functional interactions between homeodomain NKX2.1 and winged helix/forkhead FOXA1 in lung epithelial cells. Mol Cell Biol 27, 2155-2165, doi:10.1128/MCB.01133-06 (2007).

25 Kaestner, K. H. The FoxA factors in organogenesis and differentiation. Curr Opin Genet Dev 20, 527-532, doi:10.1016/j.gde.2010.06.005 (2010).

26 Bochkis, I. M. et al. Genome-wide location analysis reveals distinct transcriptional circuitry by paralogous regulators Foxa1 and Foxa2. PLoS Genet 8, e1002770, doi:10.1371/journal.pgen.1002770 (2012).

27 Wan, H. et al. Compensatory roles of Foxa1 and Foxa2 during lung morphogenesis. J Biol Chem 280, 13809-13816, doi:10.1074/jbc.M414122200 (2005).

28 Kaestner, K. H. The making of the liver: developmental competence in foregut endoderm and induction of the hepatogenic program. Cell Cycle 4, 1146-1148, doi:10.4161/cc.4.9.2033 (2005).

29 Lee, C. S., Friedman, J. R., Fulmer, J. T. \& Kaestner, K. H. The initiation of liver development is dependent on Foxa transcription factors. Nature 435, 944-947, doi:10.1038/nature03649 (2005).

30 Gao, N. et al. Dynamic regulation of Pdx1 enhancers by Foxa1 and Foxa2 is essential for pancreas development. Genes Dev 22, 3435-3448, doi:10.1101/gad.1752608 (2008).

31 Besnard, V., Wert, S. E., Hull, W. M. \& Whitsett, J. A. Immunohistochemical localization of Foxa1 and Foxa2 in mouse embryos and adult tissues. Gene Expr Patterns 5, 193-208, doi:10.1016/j.modgep.2004.08.006 (2004). Paranjapye, A., Mutolo, M. J., Ebron, J. S., Leir, S. H. \& Harris, A. The FOXA1 transcriptional network coordinates key functions of primary human airway epithelial cells. Am J Physiol Lung Cell Mol Physiol 319, L126-I136, doi:10.1152/ajplung.00023.2020 (2020).

33 Gao, B., Xie, W., Wu, X., Wang, L. \& Guo, J. Functionally analyzing the important roles of hepatocyte nuclear factor 3 (FoxA) in tumorigenesis. Biochim Biophys Acta Rev Cancer 1873, 188365, doi:10.1016/j.bbcan.2020.188365 (2020).

34 Bernardo, G. M. \& Keri, R. A. FOXA1: a transcription factor with parallel functions in development and cancer. Biosci Rep 32, 113-130, doi:10.1042/BSR20110046 (2012).

35 Robinson, J. L., Holmes, K. A. \& Carroll, J. S. FOXA1 mutations in hormone-dependent cancers. Front Oncol 3, 20, doi:10.3389/fonc.2013.00020 (2013).

36 Teng, M., Zhou, S., Cai, C., Lupien, M. \& He, H. H. Pioneer of prostate cancer: past, present and the future of FOXA1. Protein \& Cell 12, 29-38, doi:10.1007/s13238-020-00786-8 (2021).

37 Cha, Y. J. \& Shim, H. S. Biology of invasive mucinous adenocarcinoma of the lung. Transl Lung Cancer Res 6, 508-512, doi:10.21037/tlcr.2017.06.10 (2017).

38 Boggaram, V. Thyroid transcription factor-1 (TTF-1/Nkx2.1/TITF1) gene regulation in the lung. Clin Sci (Lond) 116, 27-35, doi:10.1042/CS20080068 (2009). 
39 Weir, B. A. et al. Characterizing the cancer genome in lung adenocarcinoma. Nature 450, 893898, doi:10.1038/nature06358 (2007).

40 Hight, S. K. et al. An in vivo functional genomics screen of nuclear receptors and their coregulators identifies FOXA1 as an essential gene in lung tumorigenesis. Neoplasia 22, 294310, doi:10.1016/j.neo.2020.04.005 (2020).

41 Basseres, D. S. et al. Frequent downregulation of the transcription factor Foxa2 in lung cancer through epigenetic silencing. Lung Cancer 77, 31-37, doi:10.1016/j.lungcan.2012.01.011 (2012).

42 Bejarano, P. A. et al. Surfactant proteins and thyroid transcription factor-1 in pulmonary and breast carcinomas. Mod Pathol 9, 445-452 (1996).

43 Sund, N. J. et al. Hepatocyte nuclear factor 3beta (Foxa2) is dispensable for maintaining the differentiated state of the adult hepatocyte. Mol Cell Biol 20, 5175-5183 (2000).

44 Young, N. P., Crowley, D. \& Jacks, T. Uncoupling cancer mutations reveals critical timing of p53 loss in sarcomagenesis. Cancer Res 71, 4040-4047, doi:10.1158/0008-5472.CAN-104563 (2011).

45 Lee, C. L. et al. Generation of primary tumors with Flp recombinase in FRT-flanked p53 mice. Dis Model Mech 5, 397-402, doi:10.1242/dmm.009084 (2012).

46 Schonhuber, N. et al. A next-generation dual-recombinase system for time- and host-specific targeting of pancreatic cancer. Nat Med 20, 1340-1347, doi:10.1038/nm.3646 (2014).

47 Caswell, D. R. et al. Obligate Progression Precedes Lung Adenocarcinoma Dissemination. Cancer Discovery 4, 781-789, doi:10.1158/2159-8290.cd-13-0862 (2014).

48 Dankort, D. et al. A new mouse model to explore the initiation, progression, and therapy of BRAFV600E-induced lung tumors. Genes Dev 21, 379-384, doi:10.1101/gad.1516407 (2007).

49 Camolotto, S. A. et al. FoxA1 and FoxA2 drive gastric differentiation and suppress squamous identity in NKX2-1-negative lung cancer. Elife 7, doi:10.7554/eLife.38579 (2018).

50 Yang, D. et al. Lineage Recording Reveals the Phylodynamics, Plasticity and Paths of Tumor Evolution. bioRxiv, 2021.2010.2012.464111, doi:10.1101/2021.10.12.464111 (2021).

51 Travaglini, K. J. et al. A molecular cell atlas of the human lung from single-cell RNA sequencing. Nature 587, 619-625, doi:10.1038/s41586-020-2922-4 (2020).

52 Du, Y., Guo, M., Whitsett, J. A. \& Xu, Y. 'LungGENS': a web-based tool for mapping single-cell gene expression in the developing lung. Thorax 70, 1092-1094, doi:10.1136/thoraxjnl-2015207035 (2015).

53 Strunz, M. et al. Alveolar regeneration through a Krt8+ transitional stem cell state that persists in human lung fibrosis. Nat Commun 11, 3559, doi:10.1038/s41467-020-17358-3 (2020).

54 Kobayashi, Y. et al. Persistence of a regeneration-associated, transitional alveolar epithelial cell state in pulmonary fibrosis. Nat Cell Biol 22, 934-946, doi:10.1038/s41556-020-0542-8 (2020).

55 Choi, J. et al. Inflammatory Signals Induce AT2 Cell-Derived Damage-Associated Transient Progenitors that Mediate Alveolar Regeneration. Cell Stem Cell 27, 366-382 e367, doi:10.1016/j.stem.2020.06.020 (2020).

56 Moses, M. A. et al. Molecular Mechanisms of p63-Mediated Squamous Cancer Pathogenesis. Int J Mol Sci 20, doi:10.3390/ijms20143590 (2019).

57 Tu, S. et al. MAnorm2 for quantitatively comparing groups of ChIP-seq samples. Genome Res 31, 131-145, doi:10.1101/gr.262675.120 (2021).

58 Bachurski, C. J., Yang, G. H., Currier, T. A., Gronostajski, R. M. \& Hong, D. Nuclear factor I/thyroid transcription factor 1 interactions modulate surfactant protein $\mathrm{C}$ transcription. Mol Cell Biol 23, 9014-9024, doi:10.1128/MCB.23.24.9014-9024.2003 (2003).

59 Herriges, J. C. et al. Genome-scale study of transcription factor expression in the branching mouse lung. Dev Dyn 241, 1432-1453, doi:10.1002/dvdy.23823 (2012).

60 Varma, S. et al. The transcription factors Grainyhead-like 2 and NK2-homeobox 1 form a regulatory loop that coordinates lung epithelial cell morphogenesis and differentiation. $J$ Biol Chem 287, 37282-37295, doi:10.1074/jbc.M112.408401 (2012). 
61 Zewdu, R. et al. An NKX2-1/ERK/WNT feedback loop modulates gastric identity and response to targeted therapy in lung adenocarcinoma. Elife 10, doi:10.7554/eLife.66788 (2021).

62 Feldser, D. M. et al. Stage-specific sensitivity to p53 restoration during lung cancer progression. Nature 468, 572-575, doi:10.1038/nature09535 (2010).

63 Samarasinghe, K. T. G. \& Crews, C. M. Targeted protein degradation: a promise for undruggable proteins. Cell Chem Biol, doi:10.1016/j.chembiol.2021.04.011 (2021).

64 Shai, A., Dankort, D., Juan, J., Green, S. \& McMahon, M. TP53 Silencing Bypasses Growth Arrest of BRAFV600E-Induced Lung Tumor Cells in a Two-Switch Model of Lung Tumorigenesis. Cancer Res 75, 3167-3180, doi:10.1158/0008-5472.CAN-14-3701 (2015).

65 Mollaoglu, G. et al. MYC Drives Progression of Small Cell Lung Cancer to a Variant Neuroendocrine Subtype with Vulnerability to Aurora Kinase Inhibition. Cancer Cell 31, 270285, doi:10.1016/j.ccell.2016.12.005 (2017).

66 Miyoshi, H. \& Stappenbeck, T. S. In vitro expansion and genetic modification of gastrointestinal stem cells in spheroid culture. Nature Protocols 8, 2471-2482, doi:10.1038/nprot.2013.153 (2013).

67 Han, X. et al. Transdifferentiation of lung adenocarcinoma in mice with Lkb1 deficiency to squamous cell carcinoma. Nat Commun 5, 3261, doi:10.1038/ncomms4261 (2014).

68 Shi, J. et al. Discovery of cancer drug targets by CRISPR-Cas9 screening of protein domains. Nat Biotechnol 33, 661-667, doi:10.1038/nbt.3235 (2015).

69 Adamson, B. et al. A Multiplexed Single-Cell CRISPR Screening Platform Enables Systematic Dissection of the Unfolded Protein Response. Cell 167, 1867-1882 e1821, doi:10.1016/j.cell.2016.11.048 (2016).

70 Dobin, A. et al. STAR: ultrafast universal RNA-seq aligner. Bioinformatics 29, 15-21, doi:10.1093/bioinformatics/bts635 (2013).

71 Bushnell, B. BBtools, 2021).

72 Liao, Y., Smyth, G. K. \& Shi, W. The R package Rsubread is easier, faster, cheaper and better for alignment and quantification of RNA sequencing reads. Nucleic Acids Research 47, e47e47, doi:10.1093/nar/gkz114 (2019).

73 Love, M. I., Huber, W. \& Anders, S. Moderated estimation of fold change and dispersion for RNA-seq data with DESeq2. Genome Biol 15, 550, doi:10.1186/s13059-014-0550-8 (2014).

74 Yu, G., Wang, L. G. \& He, Q. Y. ChIPseeker: an R/Bioconductor package for ChIP peak annotation, comparison and visualization. Bioinformatics 31, 2382-2383, doi:10.1093/bioinformatics/btv145 (2015). 


\section{FIGURES}

\section{FIGURE 1}
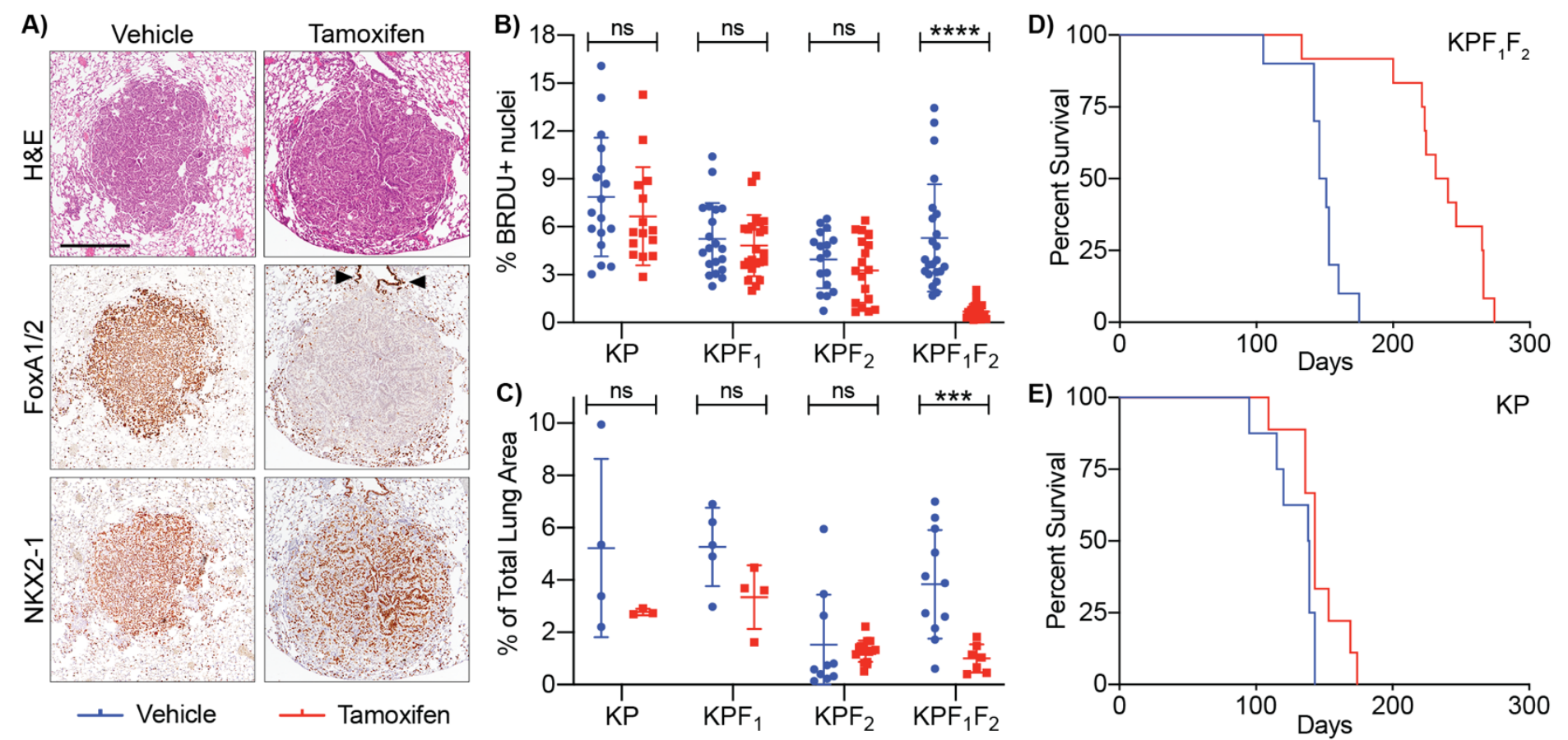

Figure 1: FoxA1/2 are required for growth of NKX2-1-positive lung adenocarcinoma in vivo

A) Representative images of $\mathrm{KPF}_{1} \mathrm{~F}_{2}$ tumors (i.e. in Kras ${ }^{\text {FSF-G12D/+} ; ~ T r p 53^{\text {Frt/Frt; Rosa26 }}}{ }^{\text {FSF-CreERT2; Foxa1 }}{ }^{\text {F/F; }}$; Foxa2 ${ }^{F / F}$ mice) 8 weeks post-initiation with adenoviral CMV-FIpO. Tamoxifen or vehicle was administered 6 weeks post-initiation. IHC for FoxA1/2 and NKX2-1 and Hematoxylin and Eosin (H\&E) staining shown. Arrowheads in center right panel mark FoxA1/2-positive normal bronchiolar epithelium, confirming tumorspecific loss of FoxA1/2 (scale bar $1000 \mu \mathrm{m}$ ).

B) BRDU IHC quantification of individual $\mathrm{KP}, \mathrm{KPF}_{1}, \mathrm{KPF}_{2}$, and $\mathrm{KPF}_{1} \mathrm{~F}_{2}$ tumors initiated with adenoviral CMVFlpO, injected with tamoxifen (red) or vehicle (blue) 6 weeks later, and sacrificed at 8 weeks ( 1 hour post BRDU injection; ${ }^{* * *} p<0.0001$, unpaired t-test)

C) Tumor burden quantification of individual mice from the experiment shown in $B\left({ }^{* * *} p=0.001014\right.$, unpaired ttest)

D) $\mathrm{KPF}_{1} \mathrm{~F}_{2}$ survival study in which tamoxifen (red, $\mathrm{n}=12$ ) or vehicle (blue, $\mathrm{n}=10$ ) was administered 10 weeks post-initiation with lentiviral EFS-FIpO (vehicle median survival of 21.21 weeks; tamoxifen median survival of 34.71 weeks; $p<0.0001$, Log-rank Mantel-Cox test). 
bioRxiv preprint doi: https://doi.org/10.1101/2021.06.29.450247; this version posted November 10, 2021. The copyright holder for this preprint (which was not certified by peer review) is the author/funder. All rights reserved. No reuse allowed without permission.

E) KP survival study in which tamoxifen (red, $n=9$ ) or vehicle (blue, $n=8$ ) was administered 10 weeks postinitiation with lentiviral EFS-FIpO (vehicle median survival of 19.79 weeks; tamoxifen median survival of 20.43 weeks; $p=0.0645$, Log-rank Mantel-Cox test). 
bioRxiv preprint doi: https://doi.org/10.1101/2021.06.29.450247; this version posted November 10, 2021. The copyright holder for this preprint (which was not certified by peer review) is the author/funder. All rights reserved. No reuse allowed without permission.

\section{SUPPLEMENTAL FIGURE 1}

$\begin{array}{lll}\text { A) Human LUAD IHC } & \text { B) }\end{array}$

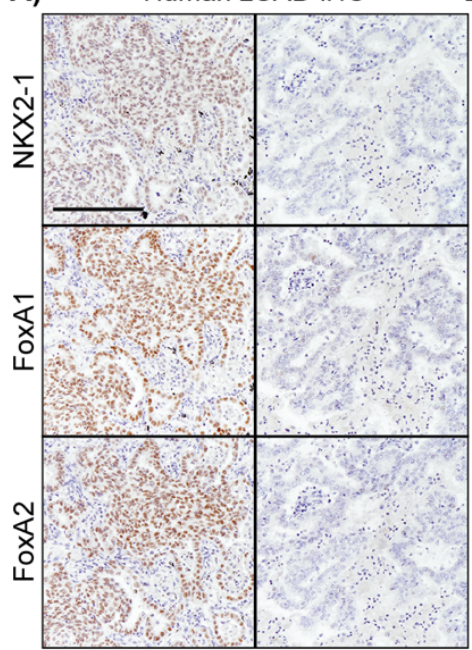

C)

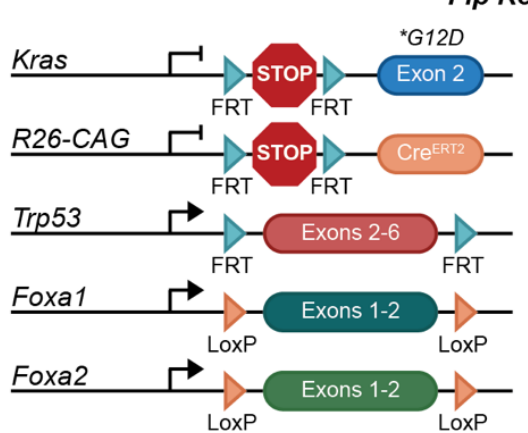

D)
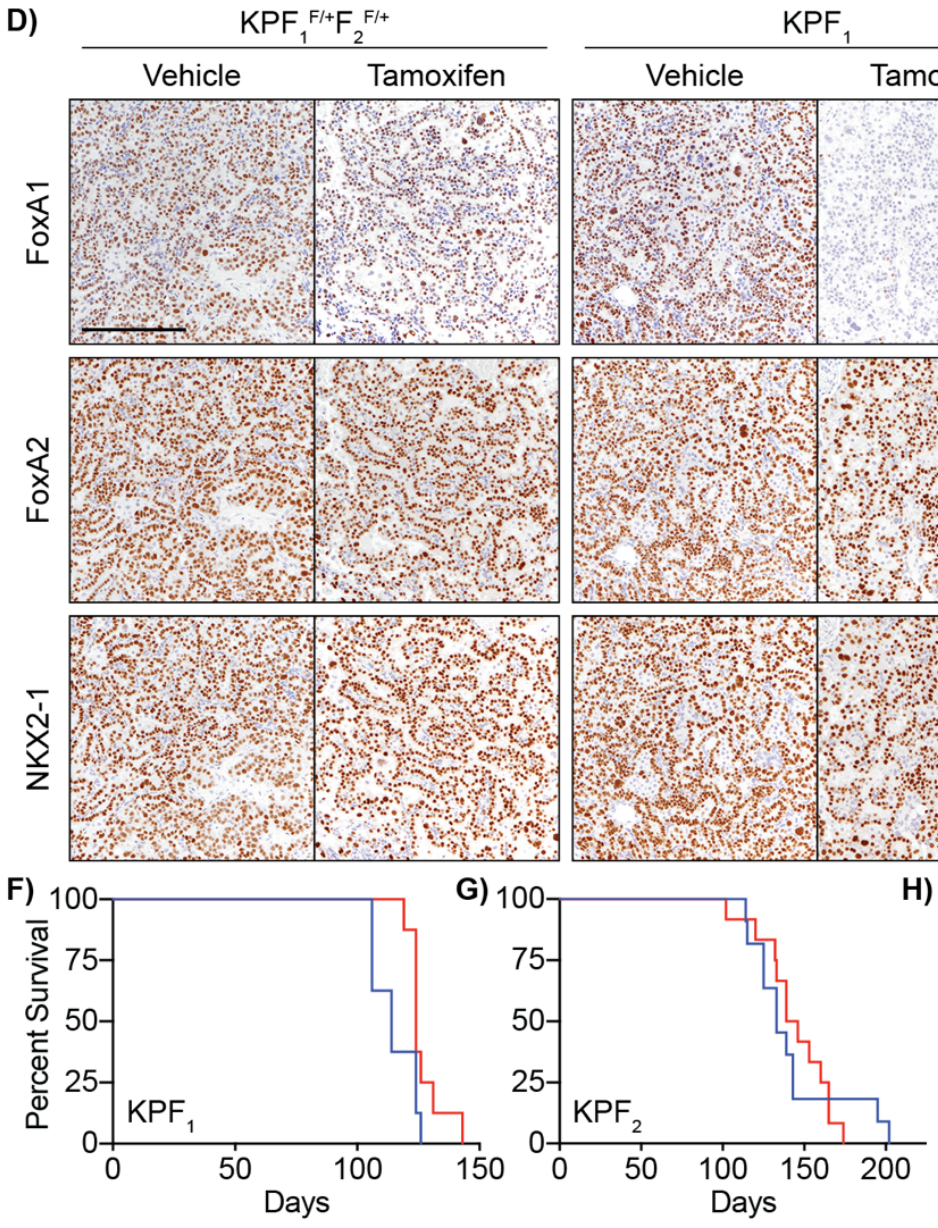

$\leftarrow$ Vehicle

NKX2-1 + NKX2-1 -
E)

FoxA1/2+
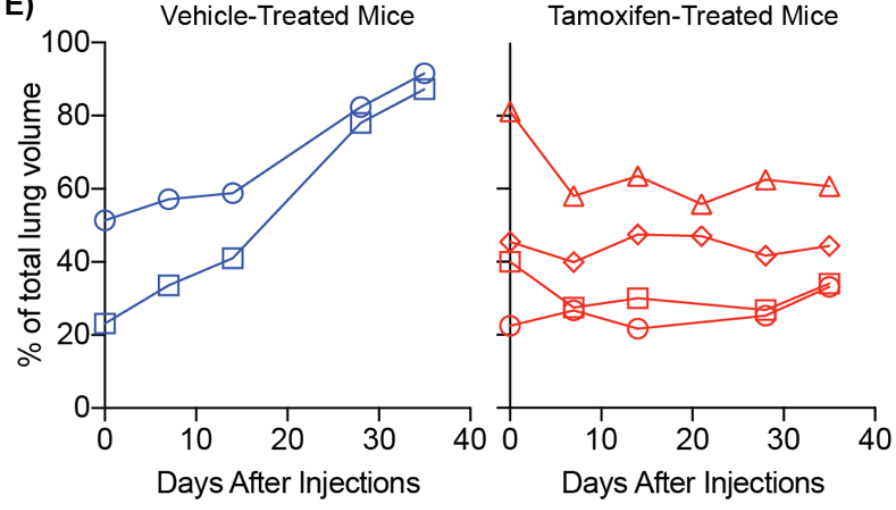

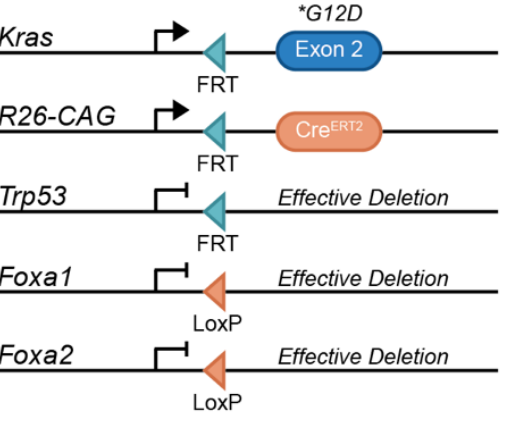

$\mathrm{KPF}_{2}$
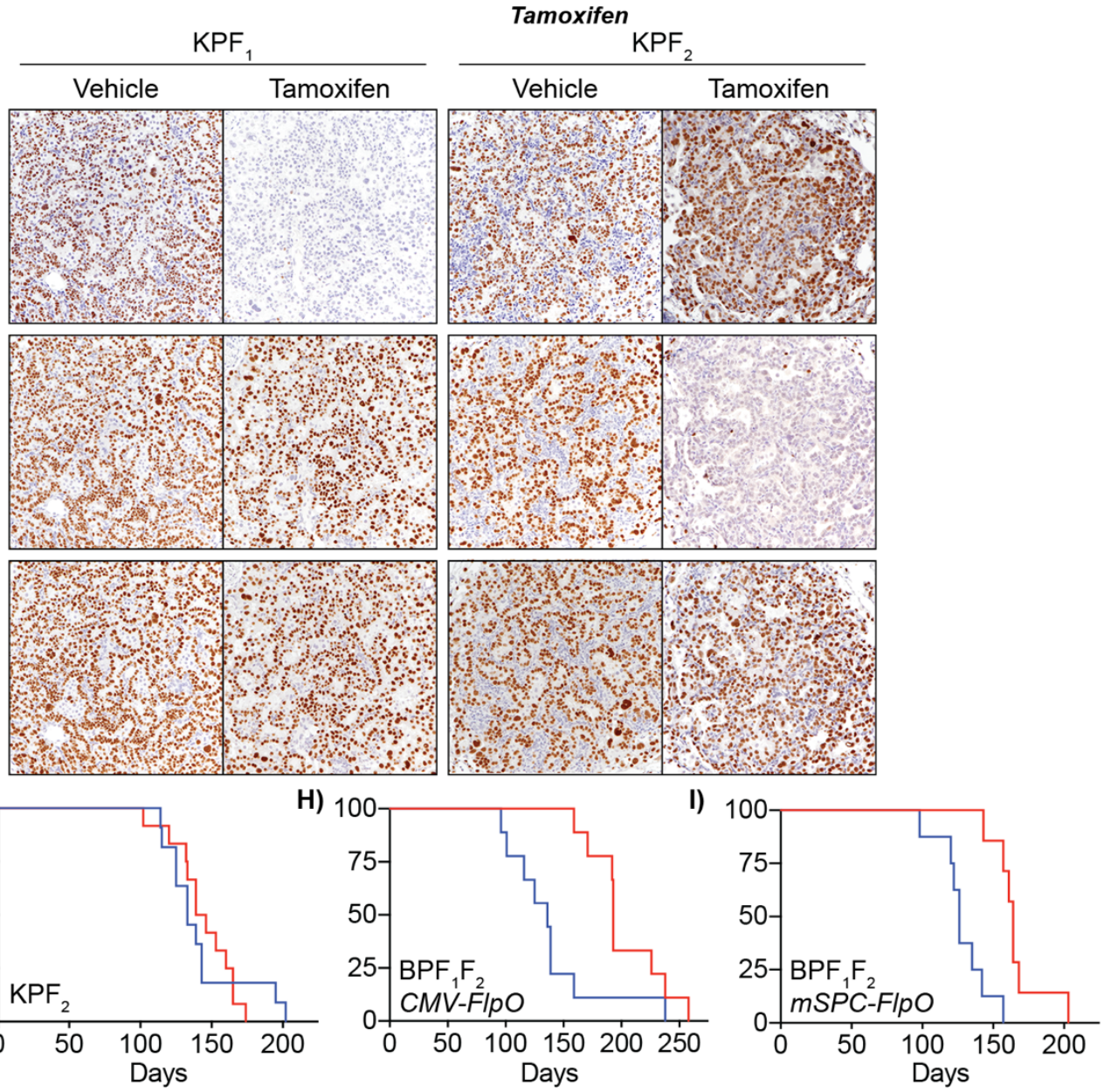


\section{Supplemental Figure 1, related to Figure 1}

A) Representative IHC images from human tissue microarrays illustrating that NKX2-1-positive tumors retain FoxA1/2 expression while NKX2-1-negative tumors can lose FoxA1/2 expression (scale bar $500 \mu \mathrm{m}$ ).

B) Histological analysis of human tumors. All NKX2-1 positive LUAD tumors evaluated ( $n=87$ ) express FoxA1 and/or FoxA2. FoxA1 and/or FoxA2 were detected in $82 \%(37 / 45)$ of NKX2-1-negative LUAD tumors evaluated, while there was no detectable FoxA1 or FoxA2 expression in 18\% (8/45) of NKX2-1-negative tumors. ( $p<0.0001$, Fisher's Exact test).

C) $\mathrm{KPF}_{1} \mathrm{~F}_{2}$ mouse model. Mice are intubated with viral FlpO recombinase, which activates the expression of oncogenic KRAS ${ }^{\mathrm{G} 12 \mathrm{D}}$ and $\mathrm{Cre}^{\mathrm{ERT2}}$ and deletes Trp53 in normal lung epithelial cells. This initiates the development of tumors in which Cre ${ }^{\mathrm{ERT} 2}$ is expressed, but sequestered in the cytoplasm. After tumors have developed, tamoxifen injection allows Cre ${ }^{\mathrm{ERT} 2}$ to enter the nucleus exclusively in tumor cells and delete Foxa1 and Foxa2.

D) Representative IHC images of $\mathrm{KPF}_{1}{ }^{\mathrm{F}+} \mathrm{F}_{2}{ }^{\mathrm{F} /+}(\mathrm{KP}), \mathrm{KPF}_{1}$, and $\mathrm{KPF}_{2}$ mice from the experiment shown in Figure 1B-C illustrating specific deletion of FoxA1 and FoxA2 (scale bar $500 \mu \mathrm{m}$ ).

E) Autochthonous lung tumors were initiated in $\mathrm{KPF}_{1} \mathrm{~F}_{2}$ mice and tracked via $\mu \mathrm{CT}$ scans monthly until there was significant detectable/traceable lung tumor volume in the three-dimensional reconstruction from each mouse (14-17 weeks post-initiation), at which point mice were treated with either vehicle ( $\mathrm{n}=2$, left panel) or tamoxifen ( $n=4$, right panel). Mice were scanned weekly to analyze tumor progression as a measurement of percentage of total lung volume.

F) $\mathrm{KPF}_{1}$ survival study in which tamoxifen $(\mathrm{red}, \mathrm{n}=8$ ) or vehicle (blue, $\mathrm{n}=8$ ) was administered 10 weeks postinitiation with lentiviral EFS-FIpO (vehicle median survival of 16.29 weeks; tamoxifen median survival of 17.71 weeks; p=0.0290, Log-rank Mantel-Cox test).

G) $\mathrm{KPF}_{2}$ survival study in which tamoxifen (red, $\mathrm{n}=12$ ) or vehicle (blue, $\mathrm{n}=11$ ) was administered 10 weeks postinitiation with lentiviral EFS-FIpO (vehicle median survival of 19.00 weeks; tamoxifen median survival of 20.36 weeks; p=0.9499, Log-rank Mantel-Cox test).

H) $B P F_{1} F_{2}$ survival study in which tamoxifen (red, $n=9$ ) or vehicle (blue, $n=9$ ) was administered 6 weeks postinitiation with Adenoviral CMV-Flp (vehicle median survival of 19.43 weeks; tamoxifen median survival of 27.57 weeks; $p=0.0098$, Log-rank Mantel-Cox test). 
bioRxiv preprint doi: https://doi.org/10.1101/2021.06.29.450247; this version posted November 10, 2021. The copyright holder for this preprint (which was not certified by peer review) is the author/funder. All rights reserved. No reuse allowed without permission.

I) $\mathrm{BPF}_{1} \mathrm{~F}_{2}$ survival study in which tamoxifen (red, $\mathrm{n}=7$ ) or vehicle (blue, $\mathrm{n}=8$ ) was administered 6 weeks postinitiation with Adenoviral SPC-Flp (vehicle median survival of 18.00 weeks; tamoxifen median survival of 23.43 weeks; $p=0.0006$, Log-rank Mantel-Cox test). 


\section{FIGURE 2}
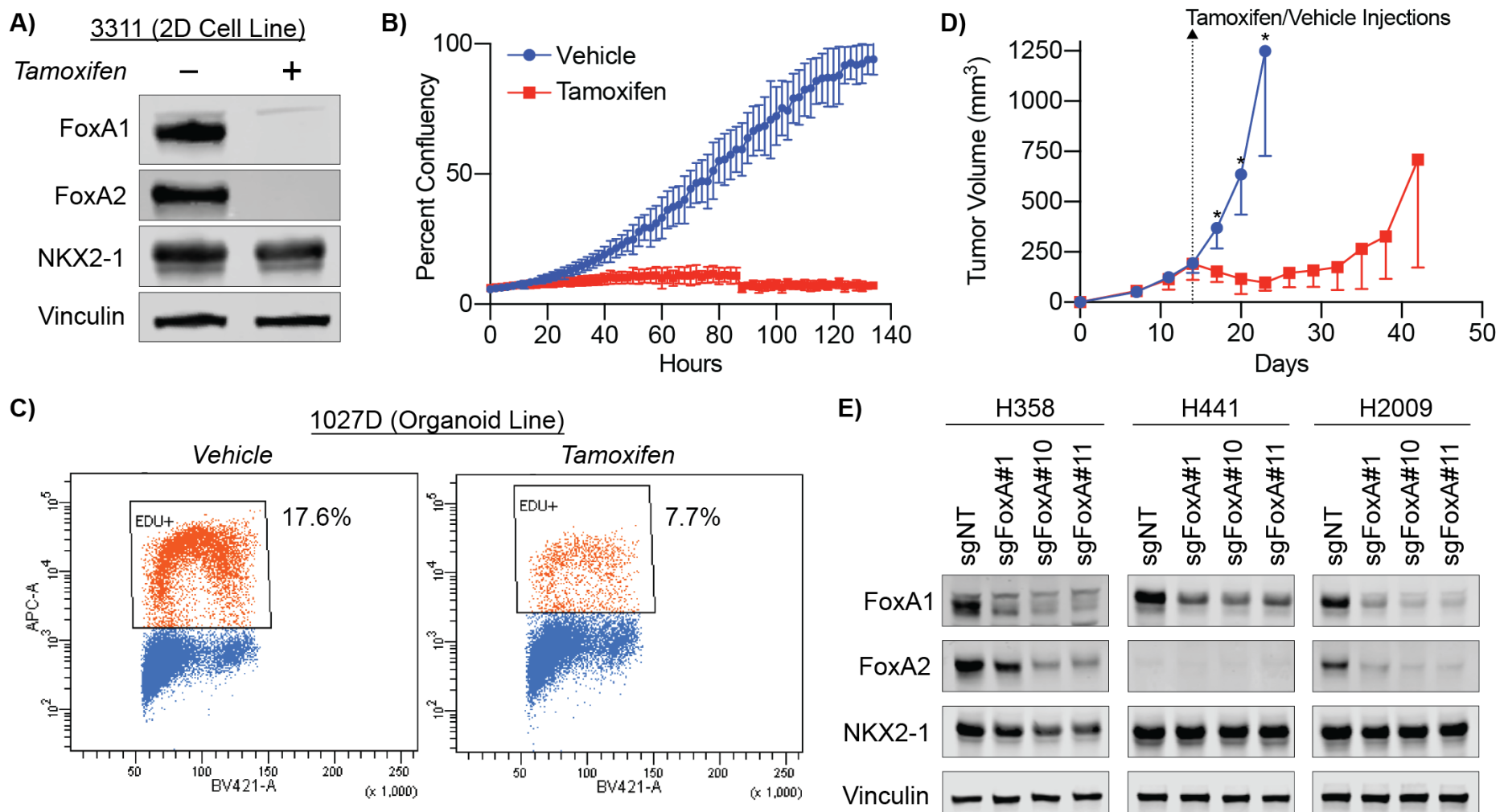

E)
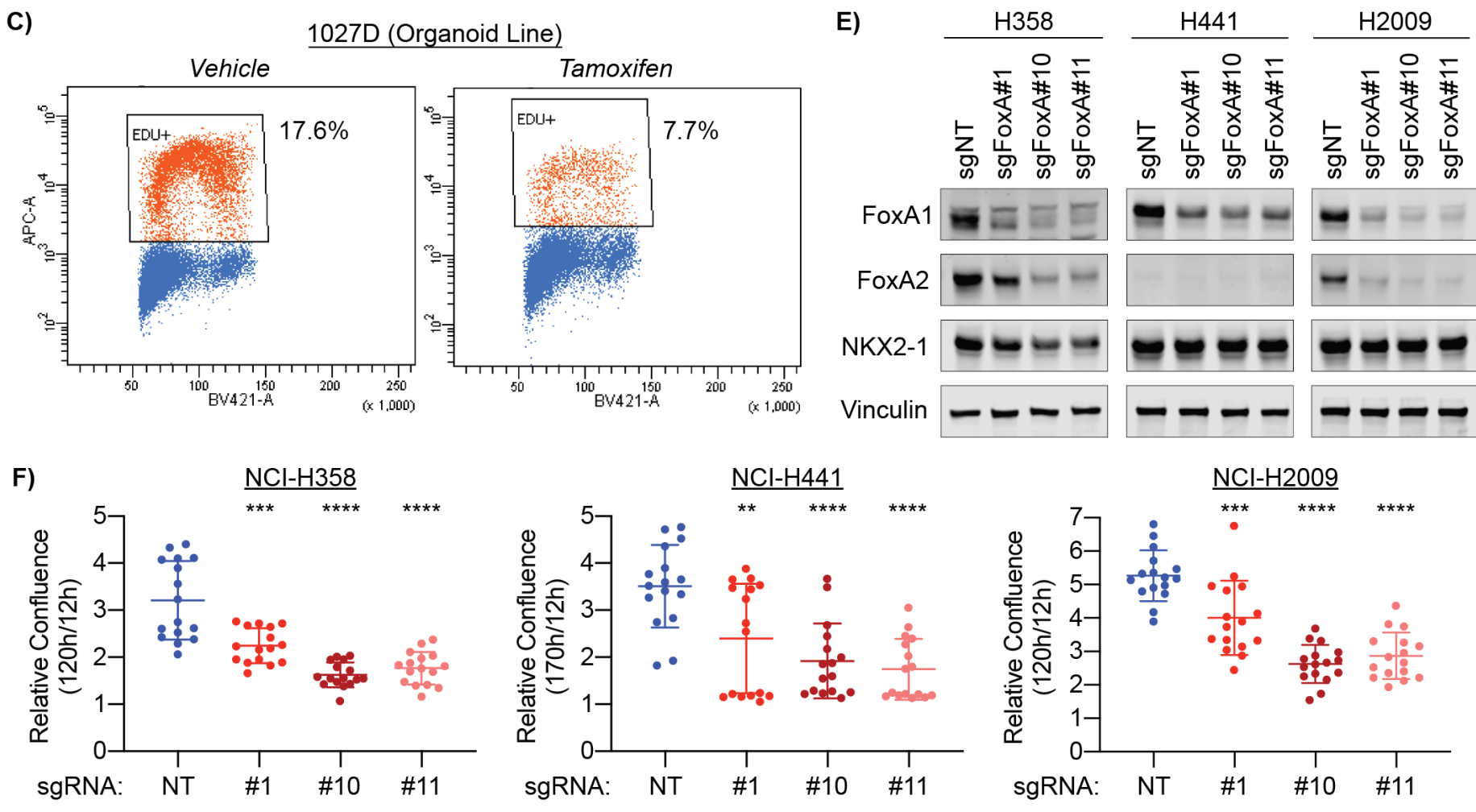

Figure 2: FoxA1/2 are required for growth of NKX2-1-positive lung adenocarcinoma in vitro.

A) Western blot of protein extract from 2D $\mathrm{KPF}_{1} \mathrm{~F}_{2}$ cell line (3311) collected 3 days post 48-hour 4-hydroxytamoxifen treatment demonstrating complete loss of FoxA1/2.

B) Incucyte-based confluency growth assay of 3311 treated with vehicle (blue) or 4-OHT (red) one week before seeding 7,000 cells/well (one biological replicate shown; representative of $n=3$ biological replicates with 8 technical replicates each; $p<0.0001$ unpaired t-test of endpoint values).

C) EdU-incorporation proliferation assay flow cytometry plots of $1027 \mathrm{D} \mathrm{KPF} F_{1} F_{2}$ organoid line one week post 72-hour treatment with vehicle (left) or 4-hydroxytamoxifen (right; BV421-A is DAPI; APC-A is EDU; representative of $n=2$ biological replicates). 
D) Subcutaneous tumors (3311 cell line) were allowed to grow to an average of $200 \mathrm{~mm}^{3}$ before treating with either vehicle $(n=5)$ or tamoxifen $(n=5)$ and progressed until one tumor from the treatment cohort surpassed $1000 \mathrm{~mm}^{3}$ (unpaired t-test day $17 p=0.0027$; day $20 p=0.0006$; day $23 p=0.0012$; representative of one of two independent experiments shown; Graph indicates mean + S.D.)

E) Western blot of protein extract from NCl-H358, NCl-H441, and $\mathrm{NCl}-\mathrm{H} 2009$ cell lines transduced with three separate lentivectors that simultaneously express unique sgRNAs targeting both FoxA1 and FoxA2 (sgFoxA\#1, sgFoxA\#10, and sgFoxA\#11), as well as a non-targeting dual sgRNA control (sgNT). Protein collected concurrently with proliferation assay seeding (6 days post-lentiviral sgRNA transduction/2 days post-selection).

F) Relative increase in percent confluence in Incucyte-based growth assay: percent confluence at 120 or 170 hours normalized to percent confluence 12 hours post-seeding ( $n=2$ biological replicates with 8 technical replicates each; unpaired t-test relative to sgNT $\left.{ }^{* *} p<0.005,{ }^{* * *} p<0.001,{ }^{* * * *} p<0.0001\right)$ 


\section{SUPPLEMENTAL FIGURE 2}

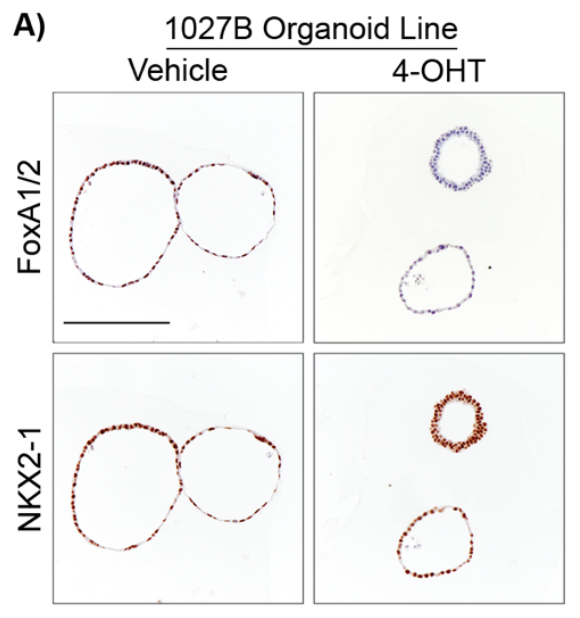

B)

Vehicle
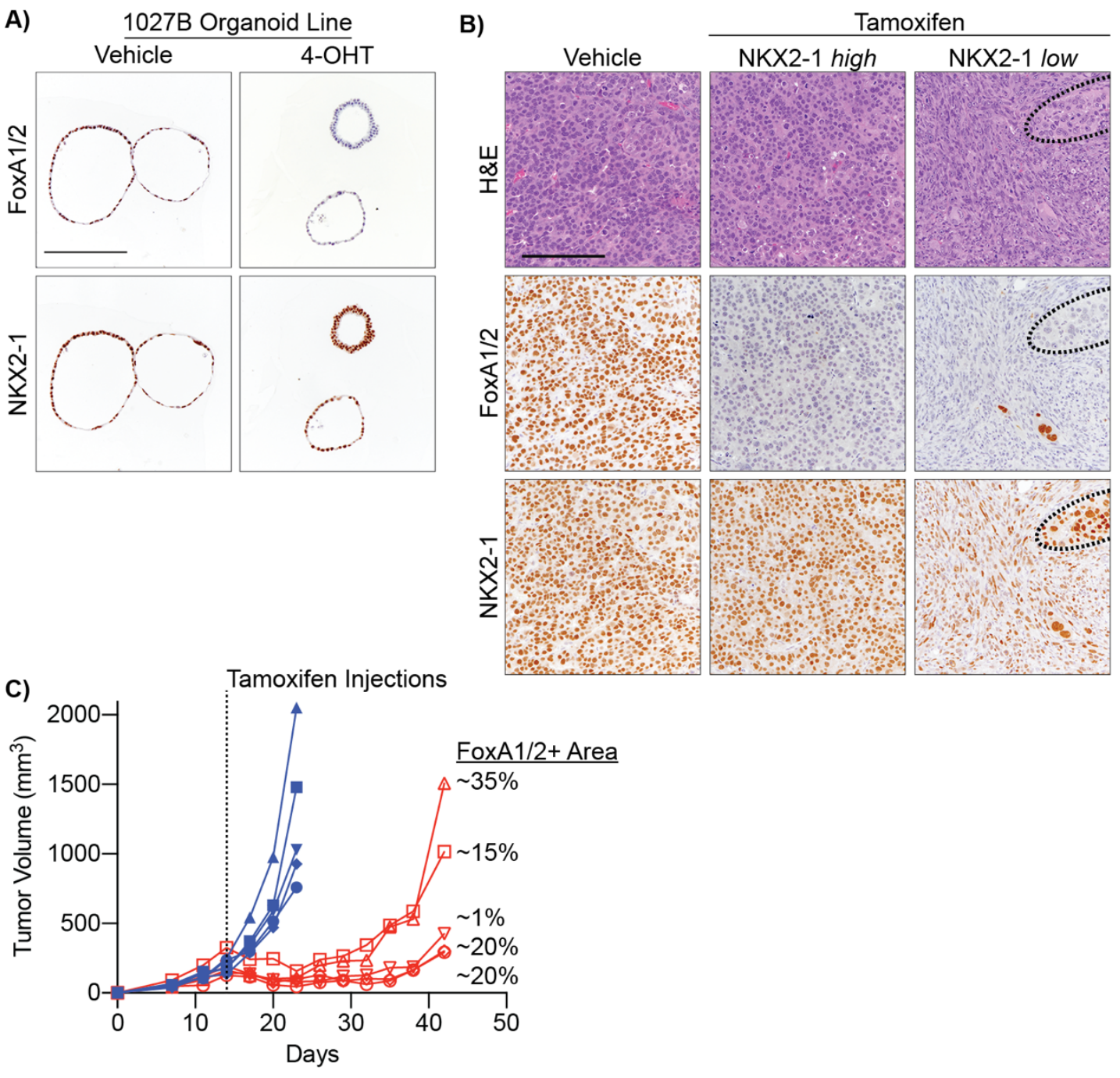

\section{Supplemental Figure 2, related to Figure 2}

A) IHC for FoxA1/2 and NKX2-1 in $\mathrm{KPF}_{1} \mathrm{~F}_{2}$ organoid line 1027B collected 3 days post 72-hour treatment with 4-OHT or vehicle demonstrating complete loss of FoxA1/2 (scale bar $500 \mu \mathrm{m}$ ).

B) Representative images of subcutaneous tumors from experiment shown in E. H\&E and IHC for FoxA1/2 and NKX2-1. Tamoxifen-treated tumors harbored two phenotypes: NKX2-1-high tumors with similar morphology to FoxA1/2-positive tumors and NKX2-1-low tumors with sarcomatoid/quasi-mesenchymal morphology (NKX2-1-high region outlined in dotted line; scale bar $500 \mu \mathrm{m}$ ).

C) Individual tumor measurements for subcutaneous tumors in Figure 2D. Approximated percent of tumor for each tamoxifen-treated mouse that retained FoxA1/2 at time of tumor collection indicated on the right. 


\section{FIGURE 3}
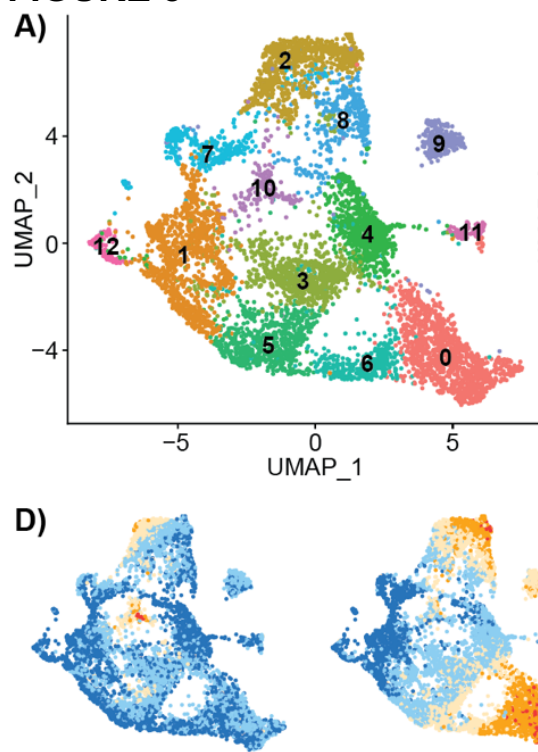

AT1/AT2

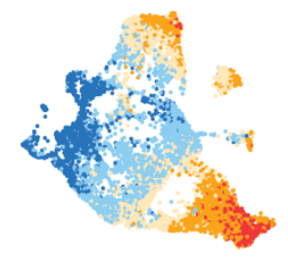

AT2
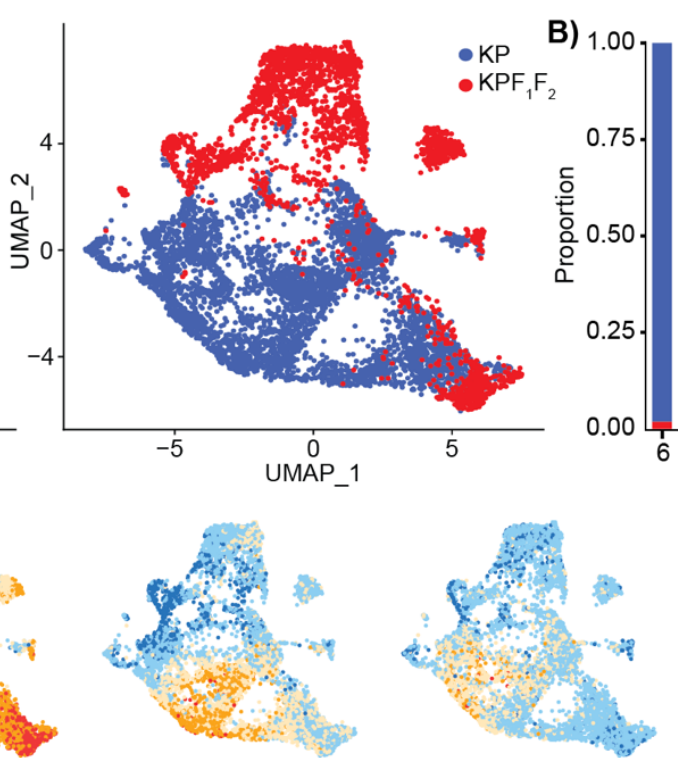

Gastric

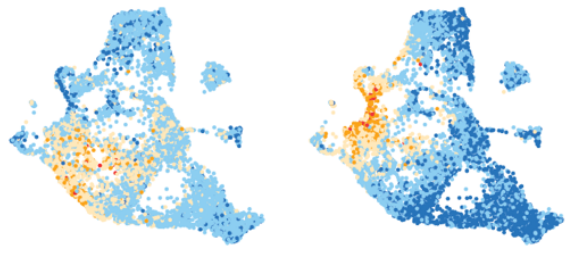

'Highly Mixed'

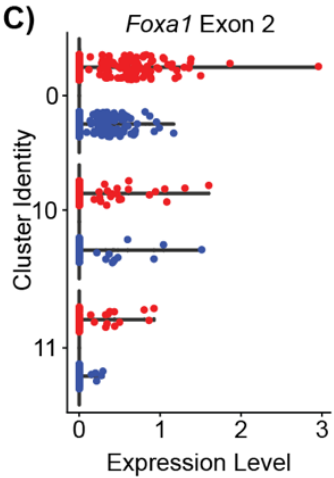

E)

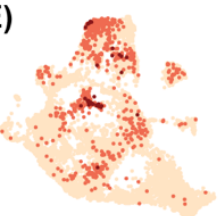

Hopx

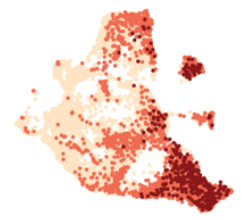

Lamp3

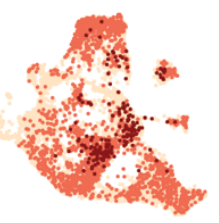

Nkx2-1

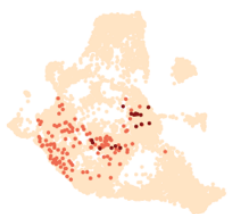

$H n f 4 \alpha$

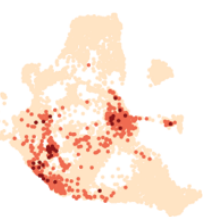

Gkn2

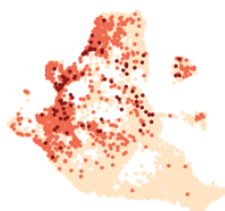

Cldn4

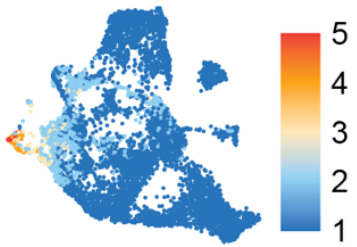

EMT

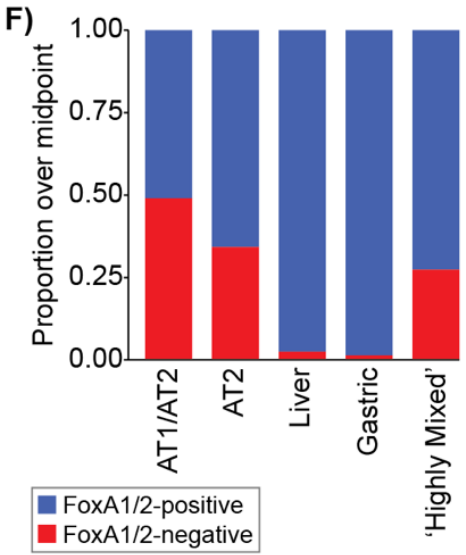

G)

NKX2-1

J) pro-SPB

pro-SPC

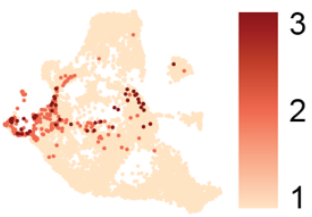

Hmga2

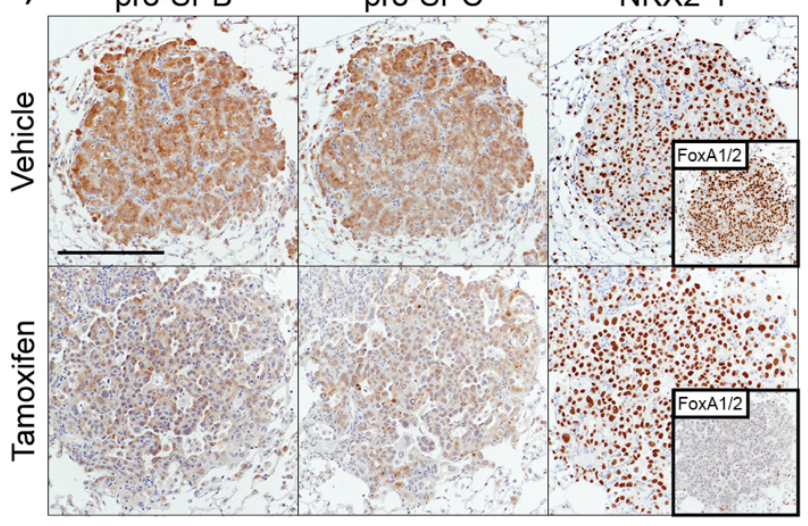

H) Gastric-
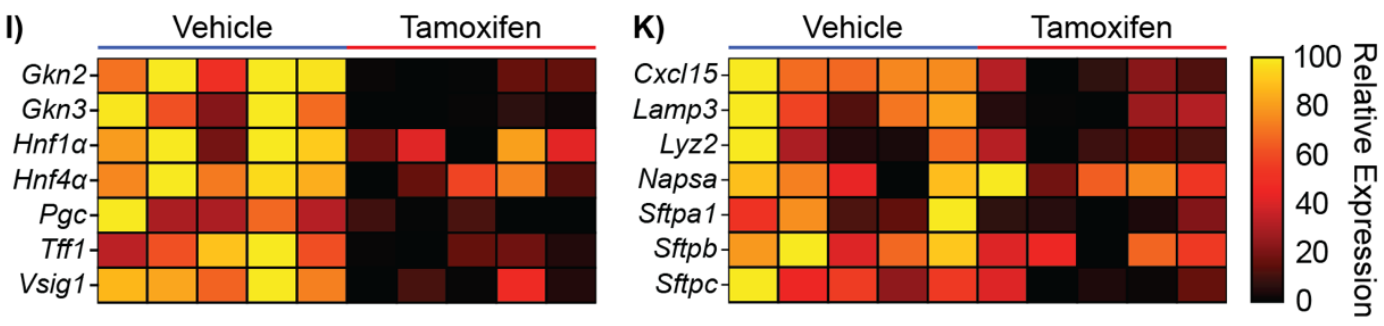

L)
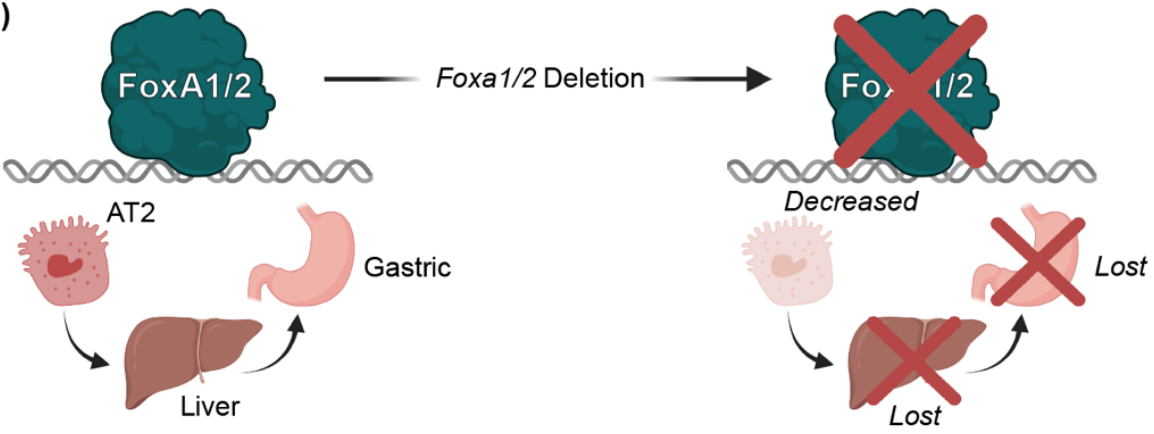


\section{Figure 3: FoxA1/2 enforce AT2 and GI-related transcriptional programs in NKX2-1-positive LUAD}

A) UMAP of cells from KP (4646 cells) and $\mathrm{KPF}_{1} \mathrm{~F}_{2}$ (2481 cells) autochthonous tumors. Left panel colored by cluster identity and right panel colored by genotype.

B) Proportion of cells in each cluster from $\mathrm{KP}$ or $\mathrm{KPF}_{1} \mathrm{~F}_{2}$ tumors.

C) Expression level of the second exon of Foxa1 that should be excised by Cre-ERT2. Detection of this exon within $\mathrm{KPF}_{1} \mathrm{~F}_{2}$ cells indicates retained expression of Foxa1/2 due to incomplete Cre-mediated recombination.

D) UMAPs of gene module scores of dual-identity transcriptional programs associated with tumor progression as defined by Marjanovic et al.

E) UMAPs of relative expression of genes corresponding to above programs (Hopx/AT1-AT2; Lamp3 and Nkx21/AT2; Hnf4a and Gkn2/Liver and Gastric; Cldn4/Highly Mixed; Hmga2/EMT).

F) Proportion of FoxA1/2-positive and FoxA1/2-negative cells with gene module scores at a level at or over the midpoint of the range of scores for the indicated transcriptional programs.

G) Representative IHC images of gastric transcription factor HNF4 $\alpha$ and pulmonary transcription factor NKX21 in FoxA1/2-positive and FoxA1/2-negative tumors, illustrating the co-expression of both endodermal and pulmonary transcription factors in KP tumors and the loss of the gastric lineage upon Foxa1/2 deletion (scale bar $500 \mu \mathrm{m})$.

H) Normalized Enrichment Score (NES) as determined by GSEA of organoid RNAseq for AT2, AT1/AT2, and Gl-related transcriptional programs as defined by Marjanovic et al, illustrating that the AT2, Liver, Gastric, and $\mathrm{Gl}$ transcriptional programs are all enriched in FoxA1/2-positive organoids. Positive NES indicates enrichment in FoxA1/2-positive organoids and negative NES indicates enrichment in FoxA1/2-negative organoids.

I) Relative expression of select gastric marker genes in FoxA1/2-positive (vehicle) and FoxA1/2-negative (tamoxifen) organoid cultures.

J) Representative IHC images of AT2 markers pro-SPB, pro-SPC, and pulmonary transcription factor NKX2-1 in FoxA1/2-positive and FoxA1/2-negative tumors, illustrating the decrease in AT2-like lineage upon Foxa1/2 deletion (scale bar $500 \mu \mathrm{m}$ ).

K) Relative expression of select AT2-specific genes in FoxA1/2-positive (vehicle) and FoxA1/2-negative (tamoxifen) organoid cultures. 
bioRxiv preprint doi: https://doi.org/10.1101/2021.06.29.450247; this version posted November 10, 2021. The copyright holder for this preprint (which was not certified by peer review) is the author/funder. All rights reserved. No reuse allowed without permission.

L) In summary, FoxA1/2 are required for the full expression of AT2, gastro-intestinal, and EMT identities associated with LUAD progression. Deletion of Foxa1/2 causes a distinct loss of liver, gastric, and EMT differentiation states and partially decreases the AT2 transcriptional program. 
bioRxiv preprint doi: https://doi.org/10.1101/2021.06.29.450247; this version posted November 10, 2021. The copyright holder for this preprint (which was not certified by peer review) is the author/funder. All rights reserved. No reuse allowed without permission.

\section{SUPPLEMENTAL FIGURE 3}
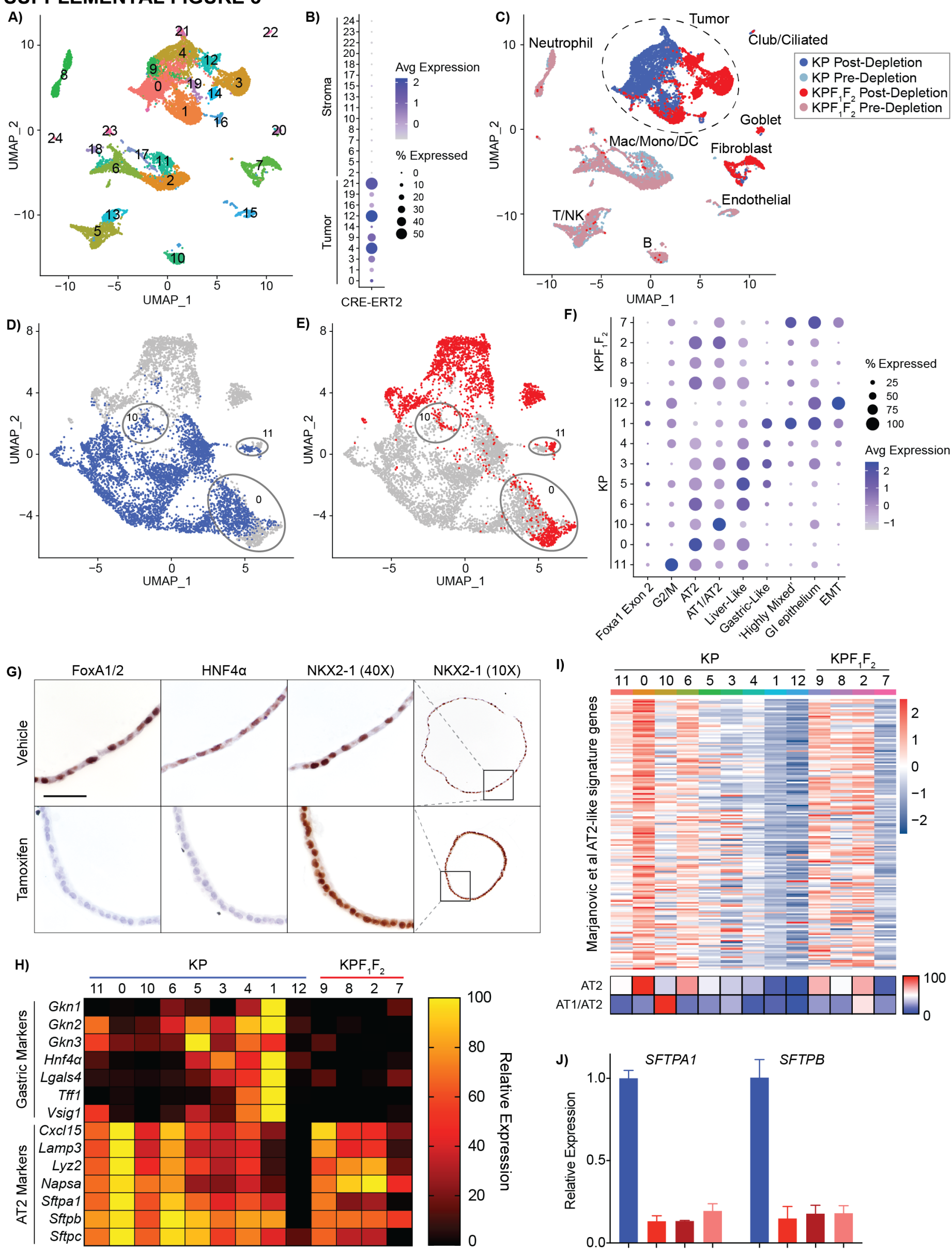
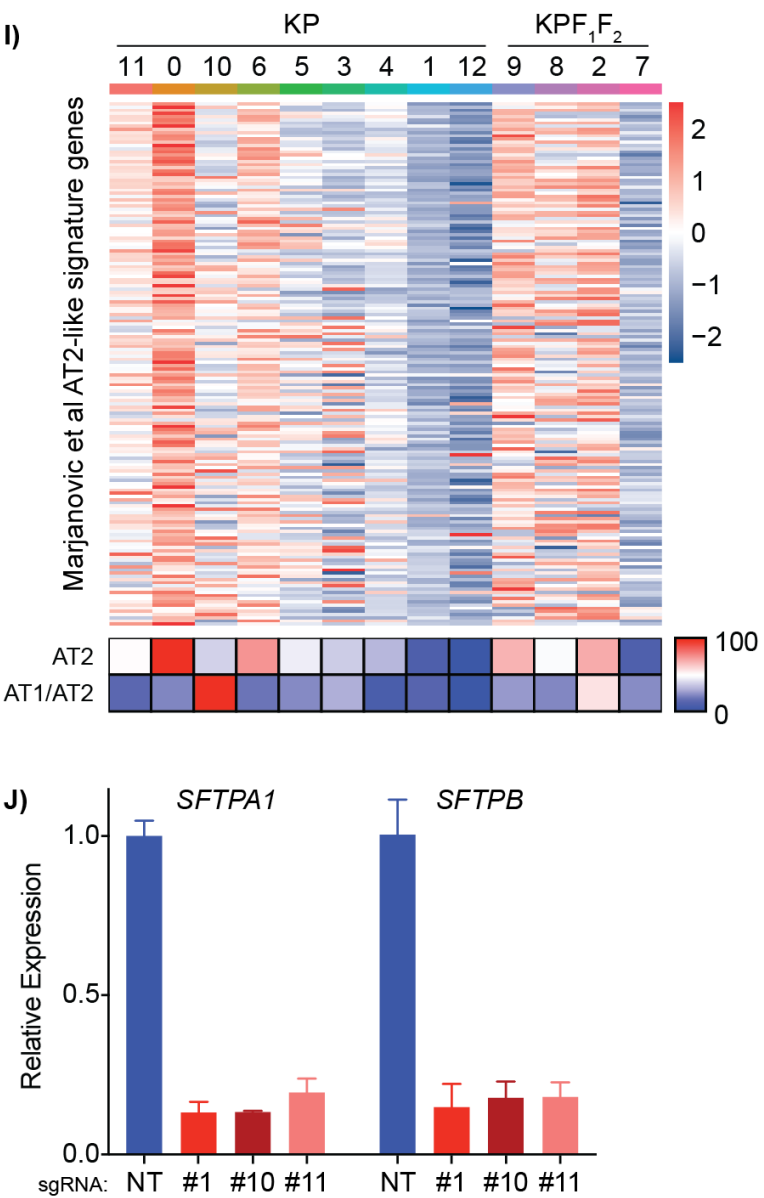


\section{Supplemental Figure 3, related to Figure 3}

A) UMAP of QC-filtered cells from KP (8269 cells) and $\mathrm{KPF}_{1} \mathrm{~F}_{2}$ (6482 cells) macro-dissected tumor regions, both pre- and post-MACS depletion of CD45+ and CD31+ cells. Cells colored by cluster identity.

B) Dot plot quantifying the level of expression and number of cells expressing CRE-ERT2, a tumor-specific transcript. CRE-ERT2 expression indicates that the cells within that cluster are tumor cells, while a complete lack of expression indicates a stromal identity.

C) UMAP shown in A with cells colored by genotype and CD45/CD31 depletion status. Clusters included in the tumor cell reclustering (Figure 3A) circled with dashed line.

D) UMAP of cells from KP (blue) and

E) $\mathrm{KPF}_{1} \mathrm{~F}_{2}$ samples (red) illustrating the level of overlap between the two genotypes in clusters 0,10 , and 11 . $\mathrm{KPF}_{1} \mathrm{~F}_{2}$ cells in these clusters retain expression of Foxa1 Exon 2 indicating incomplete recombination.

F) Dot plot quantifying Foxa1 Exon2 expression and Marjanovic program module scores in the tumor cell UMAP of Figure 3A illustrating enrichment for Foxa1 Exon2 and the mixed-lineage tumor progression within KPspecific clusters relative to $\mathrm{KPF}_{1} \mathrm{~F}_{2}$ clusters.

G) Representative IHC images of gastric transcription factor HNF4 $\alpha$ and pulmonary transcription factor NKX21 in FoxA1/2-positive and FoxA1/2-negative 1027B organoids, illustrating the loss of gastric lineage upon Foxa1/2 deletion (scale bar $100 \mu \mathrm{m}$ ).

H) Row normalized average expression levels of gastric and AT2 genes in each cluster shown in Figure 3A demonstrating a distinct loss of gastric identity markers in FoxA1/2-negative clusters $(2,7,8,9)$ relative to gastric-like KP clusters $(1,3,4,5,6)$ and a decrease in AT2 identity markers in FoxA1/2-negative clusters relative to AT2-like KP Cluster 0 .

I) Top: Heatmap representing average expression of the AT2-like signature in which each row is a gene of the AT2-like program and each column is a cluster's average expression of an individual gene. Bottom: Heatmap of average AT2 and AT1/AT2 module scores in each tumor cell cluster

J) Relative expression of SFTPA1 and SFTPB in H441 cells lines (shown in Figure 2) as determined by RTqPCR. 


\section{FIGURE 4}

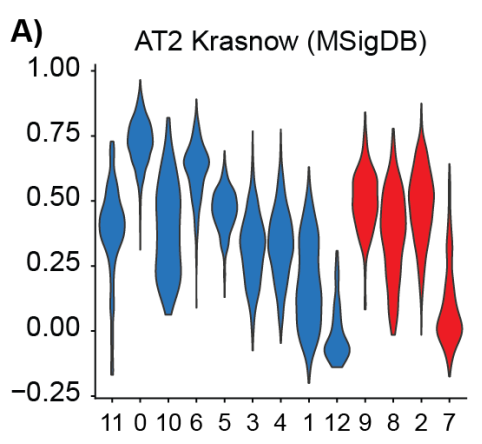

E)

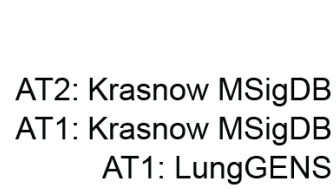

AT1: NKX2-1-dependent genes

PATS

G)

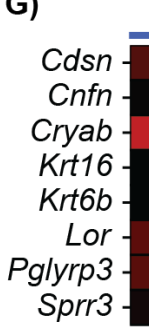

Vehicle

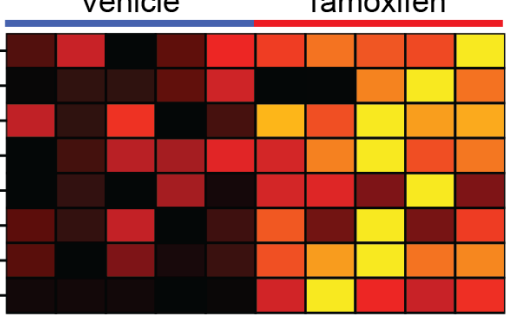

J)

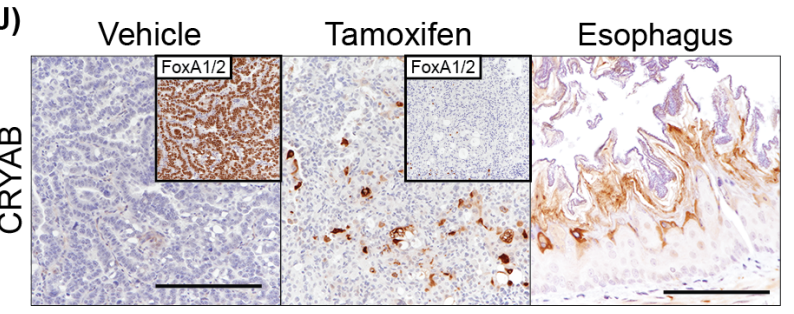

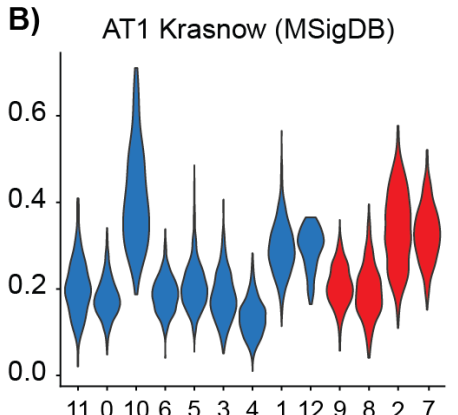

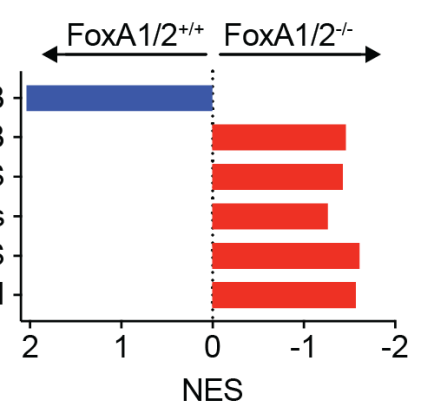

NES

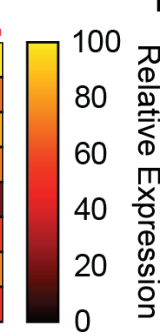

H)

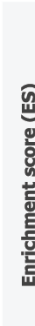

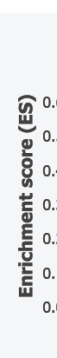

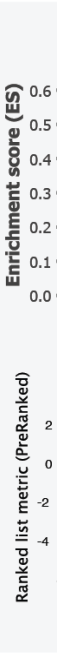

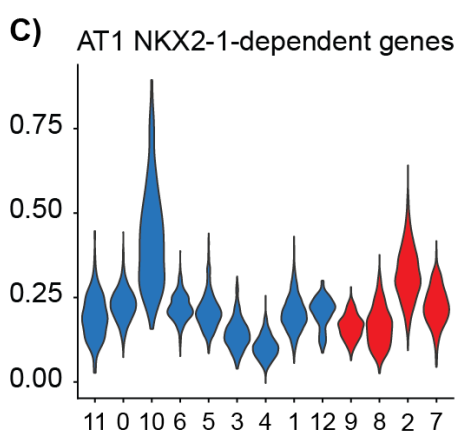

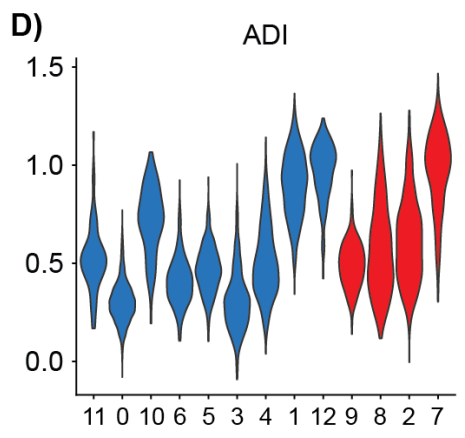

F)

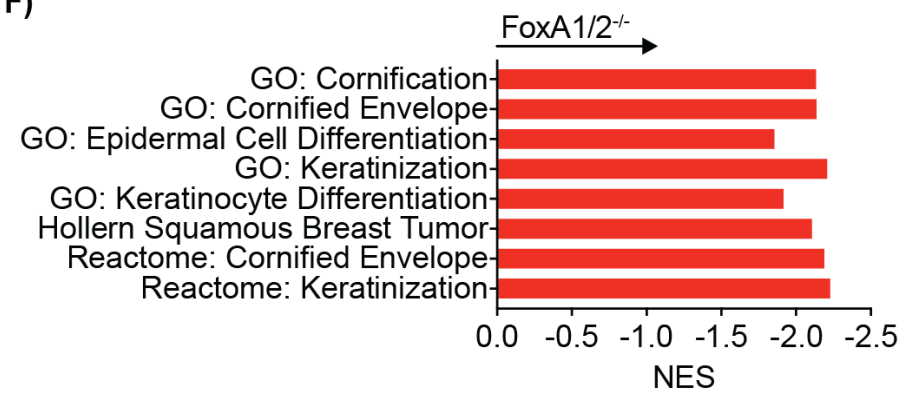

I) GOBP_KERATINOCYTE_DIFFERENTIATION GOBP_KERATINOCYTE_DIFFERENTIATION

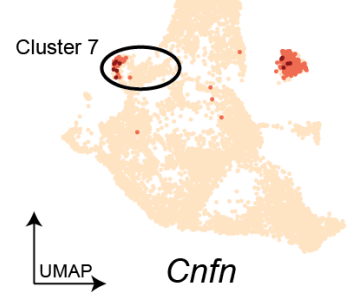
FDR: 0.000 $p=0.000$
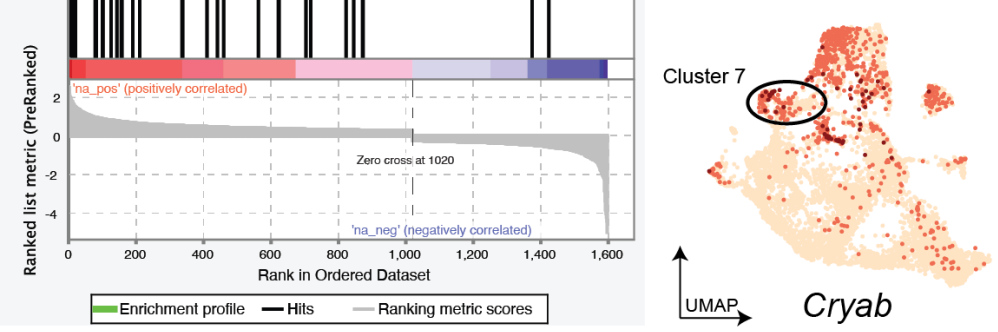

Figure 4: FoxA1/2 suppress alternative pulmonary and stratified squamous transcriptional programs in

\section{NKX2-1-positive lung adenocarcinoma}

A) Violin plot representing the expression of the Krasnow AT2 transcriptional program in each tumor cell cluster.

The $y$-axis indicates gene module score. The $x$-axis indicates cluster identity.

B) Violin plot representing the expression of the Krasnow AT1 transcriptional program in each tumor cell cluster.

C) Violin plot representing the expression of NKX2-1-dependent AT1 genes in each tumor cell cluster.

D) Violin plot representing the expression of the ADI transcriptional program in each tumor cell cluster.

E) Normalized Enrichment Score (NES) as determined by GSEA of organoid RNAseq for the above gene sets. 
F) NES of representative gene sets associated with a squamous differentiation from GSEA intersection of the differentially expressed genes identified in $\mathrm{KPF}_{1} \mathrm{~F}_{2}$ organoid RNAseq with all available MSigDB gene set collections.

G) Relative expression of SSSE genes in FoxA1/2-positive (vehicle) and FoxA1/2-negative (tamoxifen) organoid cultures.

H) GSEA showing enrichment of the gene ontology signature associated with keratinocyte differentiation in FoxA1/2-negative Cluster 7.

I) UMAPs of relative expression of SSSE gene Cornifelin and SSSE/AT1 gene that encodes Alpha CrystallinB, Cryab.

J) Representative IHC images of SSSE/AT1 marker Alpha Crystallin-B in normal SSSE (Esophagus) and FoxA1/2-positive, and FoxA1/2-negative tumors, confirming the induction of these identities upon Foxa1/2 deletion (tumor scale bar $500 \mu \mathrm{m}$, esophagus scale bar $250 \mu \mathrm{m}$ ). 


\section{SUPPLEMENTAL FIGURE 4}
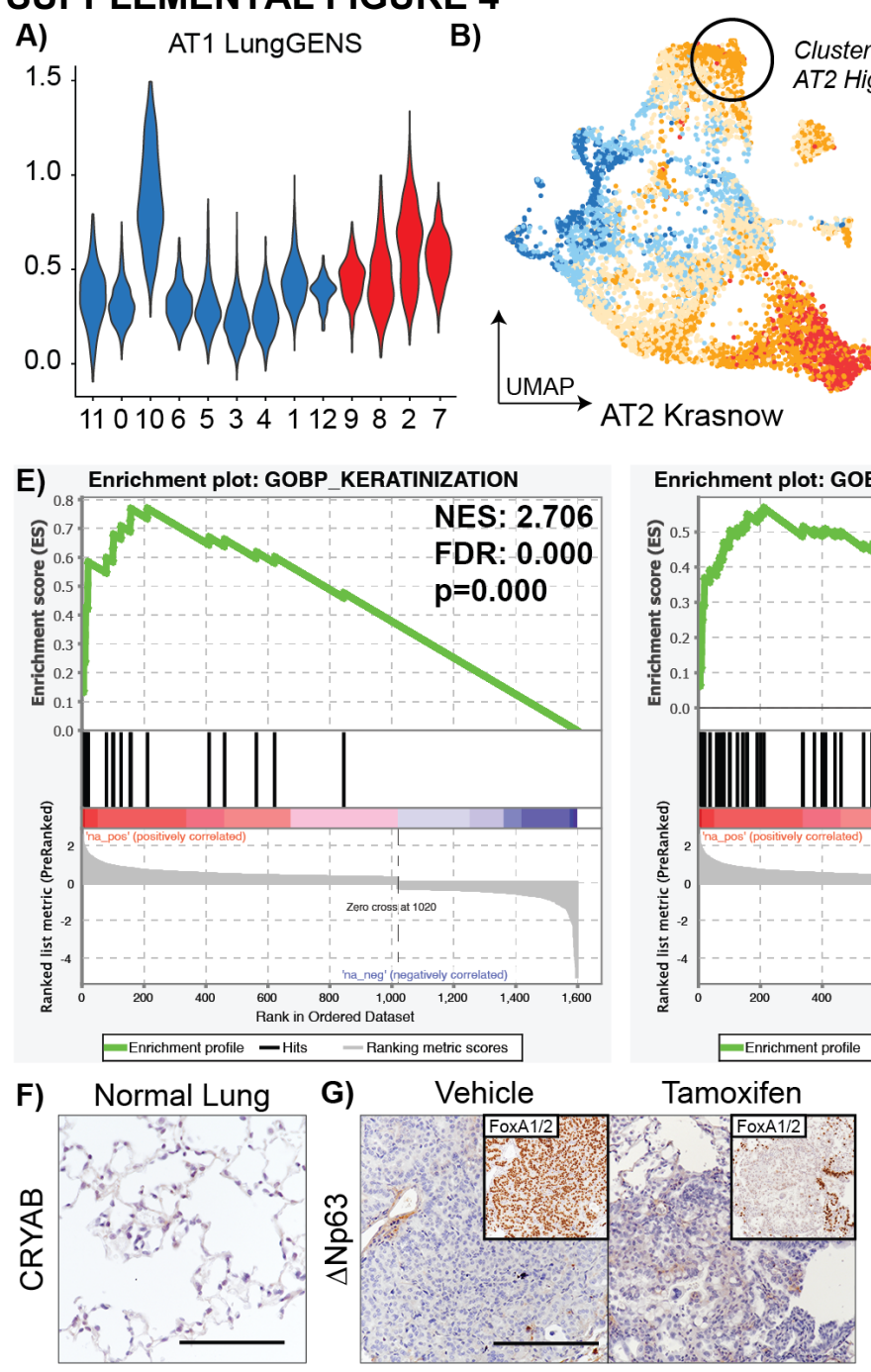

C)

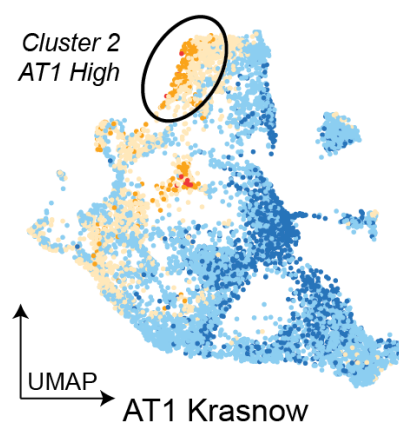

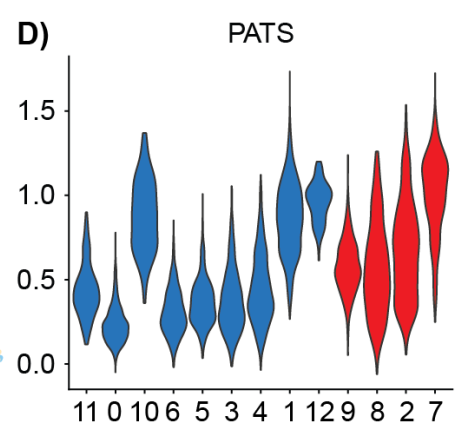
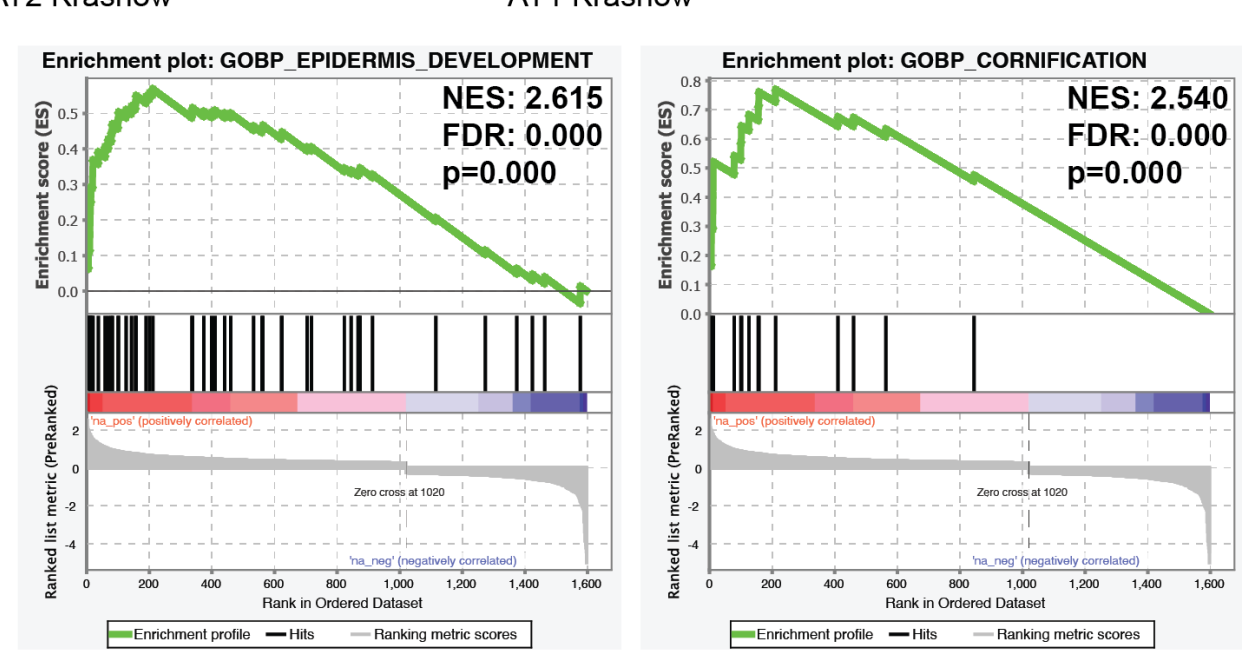

Supplemental Figure 4, related to Figure 4

A) Violin plot representing the expression of the LungGENS AT1 transcriptional program in each tumor cell cluster. The $y$-axis indicates gene module score, and the $x$-axis indicates cluster identity.

B) UMAP of gene module scores for AT2 and...

C) AT1 Krasnow gene signatures, illustrating that there is an inverse correlation between AT2 and AT1 identity within the cells that make up $\mathrm{KPF}_{1} \mathrm{~F}_{2}$-specific Cluster 2.

D) Violin plot representing the expression of the PATS transcriptional program in each tumor cell cluster.

E) GSEA showing enrichment of the gene ontology signatures associated with Keratinization, Epidermis development, and Cornification in FoxA1/2-negative Cluster 7.

F) IHC indicating that, with this antibody, we do not detect Alpha Crystallin-B in normal AT1 cells (scale bar $250 \mu \mathrm{m})$. 
bioRxiv preprint doi: https://doi.org/10.1101/2021.06.29.450247; this version posted November 10, 2021. The copyright holder for this preprint (which was not certified by peer review) is the author/funder. All rights reserved. No reuse allowed without permission.

G) Representative IHC images of $\triangle \mathrm{Np} 63$ in FoxA1/2-positive and FoxA1/2-negative tumors, illustrating the lack of expression in both tumor types (scale bar $500 \mu \mathrm{m}$ ). 


\section{FIGURE 5}

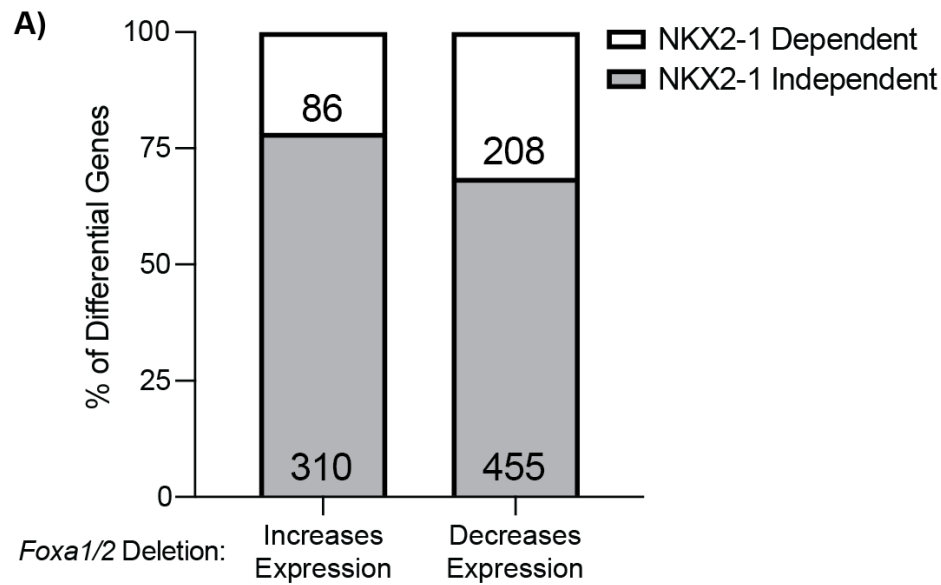

B)

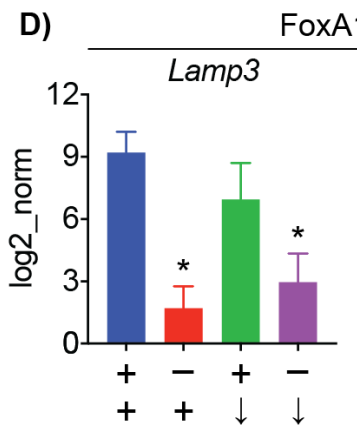

C)
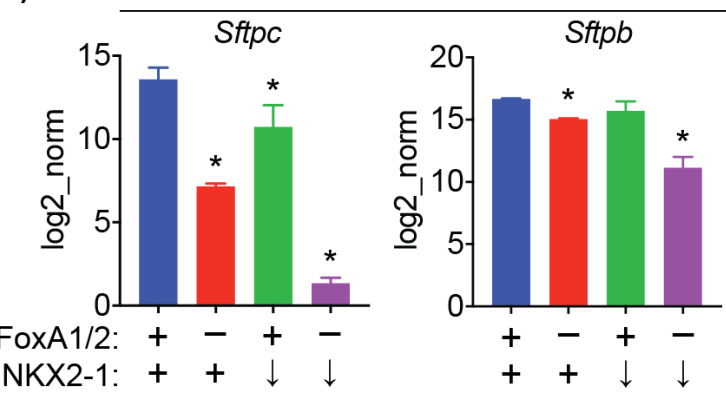

F)

Induction is dependent on NKX2-1
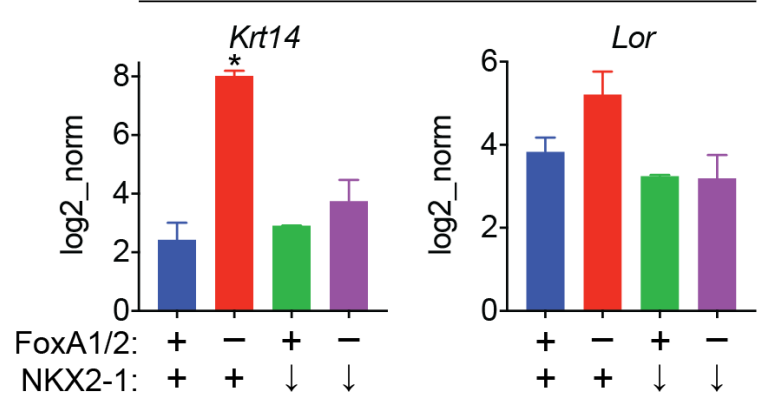

G)
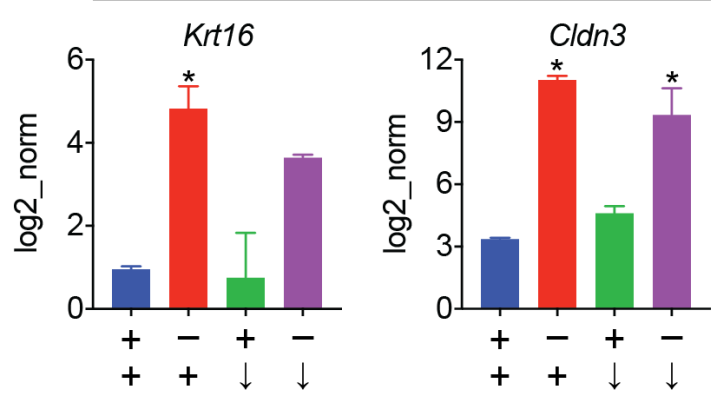

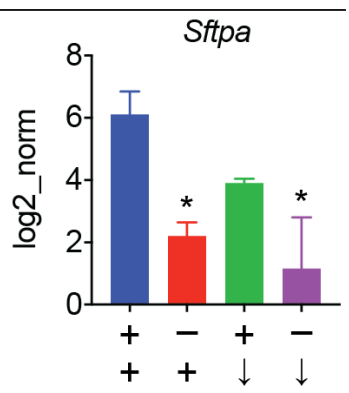

GSEA

shScramble; Tamoxifen vs shNKX2-1; Tamoxifen

$\stackrel{\text { FoxA1/2--; NKX2-1+ }}{\longrightarrow}$

AT1 Krasnow MSigDB-

AT2 Krasnow MSigDB-

GO: Epidermal Cell Differentiation-

GO: Keratinocyte Differentiation

GO: Keratinization-

GO: Cornification-

Reactome: Keratinization-

Reactome: Cornified Envelope-

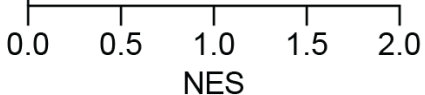

NES

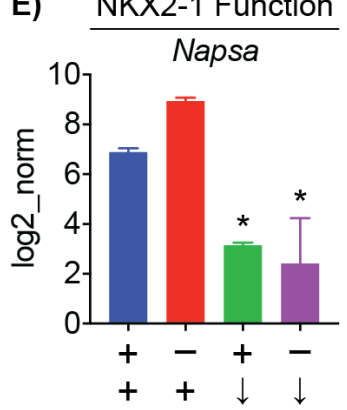

Figure 5: NKX2-1 is required for a subset of gene expression changes induced by FoxA1/2 deletion

A) Number of genes increased or decreased following Foxa1/2 deletion further categorized by NKX2-1 dependence.

B) GSEA intersecting relevant gene sets with the ranked gene list generated by differential expression analysis (DESeq2) comparing FoxA1/2-negative; NKX2-1-positive and FoxA1/2-negative; NKX2-1-low organoid cultures. Positive normalized enrichment score (NES) shows enrichment in the NKX2-1-positive cells, indicating that those gene sets are functionally dependent on NKX2-1.

C) Normalized Log2 expression values as determined by DESeq2 for AT2 genes that show additive FoxA1/2 and NKX2-1 function: Sftpc and Sftpb (decrease relative to shScramble; vehicle ${ }^{*}$ padj<0.007) 
D) Normalized Log2 expression values as determined by DESeq2 for AT2 genes primarily dependent on FoxA1/2 function: Lamp3 and Sftpa1 (decrease relative to shScramble; vehicle * padj $<2 \times 10^{-5}$ )

E) Normalized Log2 expression values as determined by DESeq2 for Napsa, an AT2 gene that is dependent on NKX2-1 and not FoxA1/2 (decrease relative to shScramble; vehicle *padj $<7.22 \times 10^{-10}$ )

F) Normalized Log2 expression values as determined by DESeq2 for SSSE genes that show dependence on NKX2-1: Krt14 and Lor (increase relative to shScramble; vehicle *padj<7.22 $\times 10^{-17}$ )

G) Normalized Log2 expression values as determined by DESeq2 for SSSE genes that are independent of NKX2-1: Krt16 and Cldn3 (increase relative to shScramble; vehicle *padj<0.006) 


\section{SUPPLEMENTAL FIGURE 5}

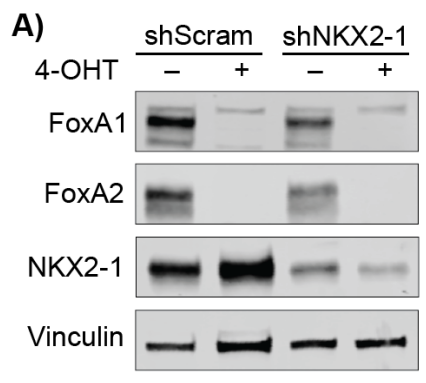

B)

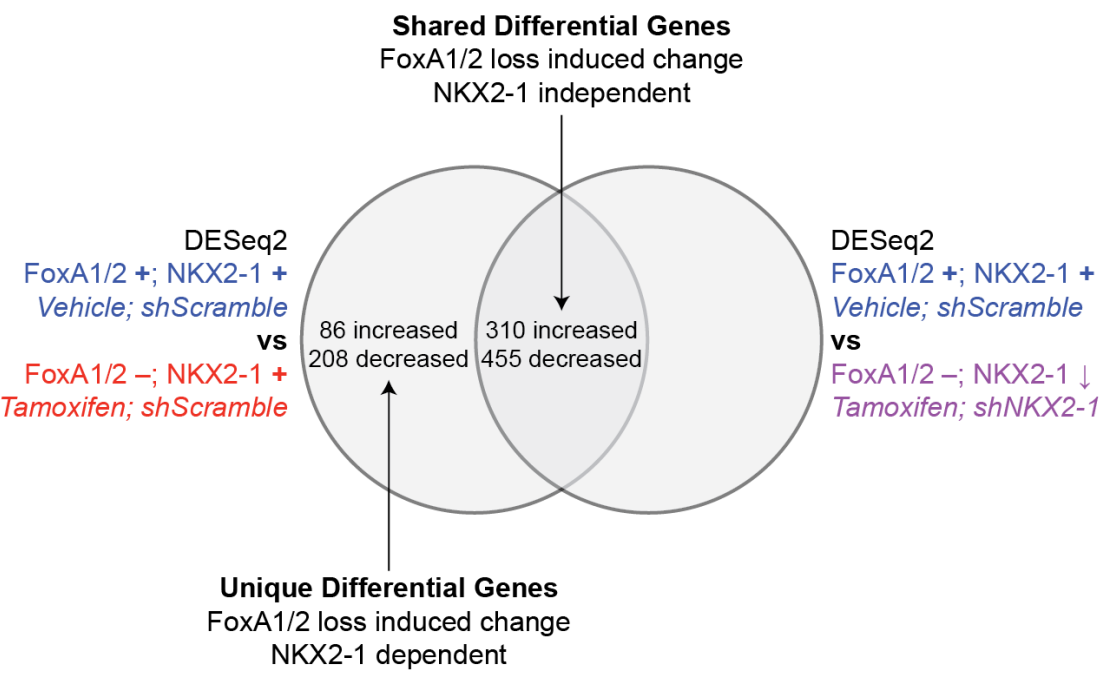

\section{Supplemental Figure 5, related to Figure 5}

A) Western blot of 1027B organoids transduced with shScramble and shNKX2-1 and then treated with 4-OHT/vehicle demonstrating successful knock down of NKX2-1.

B) Graphical representation of relevant comparisons for data shown in Figure 5. See methods section for detailed description of bioinformatic analysis. 


\section{FIGURE 6}

A)

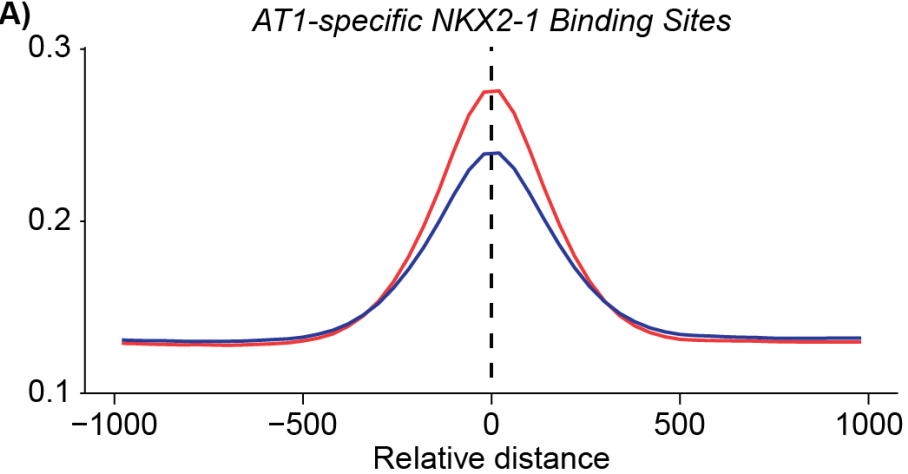

_ FoxA1/2-positive

FoxA1/2-negative

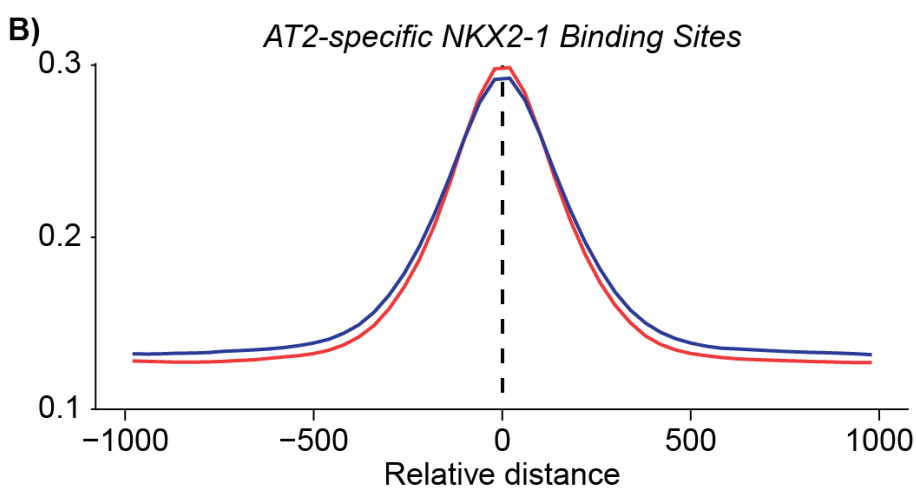

FoxA1/2-positive
FoxA1/2-negative
C) FoxA1/2-positive FoxA1/2-negative

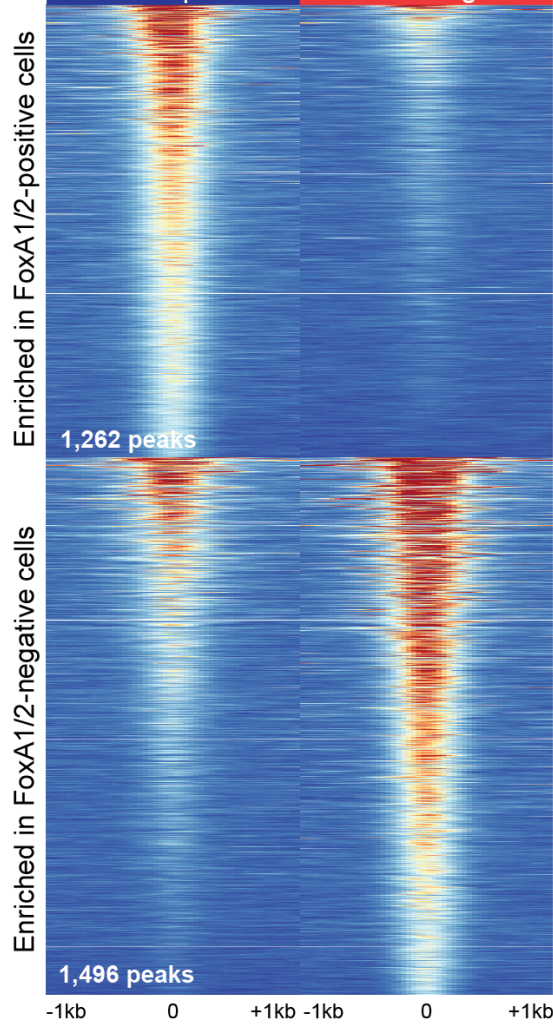

1.4 D) FoxA1/2-positive enriched motifs

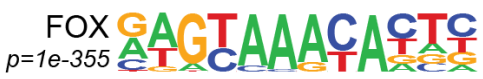
NKX TGAGTG

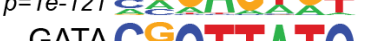
GATAC定C $p=1 \mathrm{e}-22$ ATC $\underset{p=1 e-21}{\mathrm{CEBP}}$ IACGAAA $\operatorname{sox}_{p=1 e-21} \mathrm{~A} A \mathrm{GCC} \mathrm{C} I \mathrm{C}$

E) FoxA1/2-negative enriched motifs NKX CTTGAGTGETET GATA TA $\triangle A \Lambda \mathrm{CG}$ $p=1 e-187$ AGA $A$ A

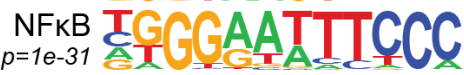
ERG (ETS) GACAAAGT NFI GTICCCAAGC MITF GAGTCACAAC

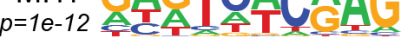
GRHL AACCGGTIT
F) No significant change enriched motifs NKX AACCAC CAA GATA ECA A A A A $p=1 e-440$ 短

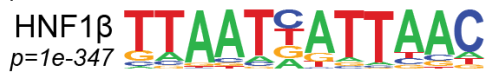
CEBP
$p=1 e-269$ FOX TGTIACACA

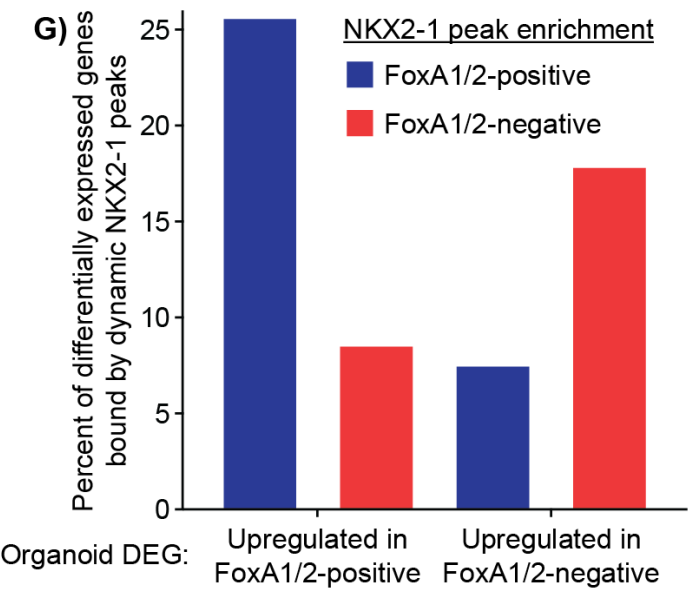

FoxA1/2:

H) Positive

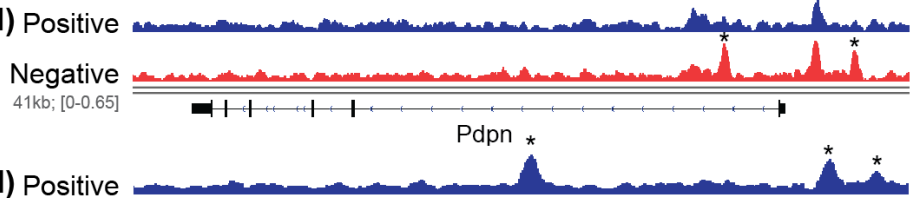

\section{Negative} 17kb; [0-0.82]

J) Positive

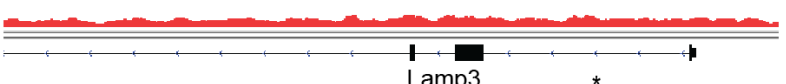

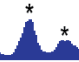

FoxA1/2:

FoxA1/2:

K) Positive Negative 47kb; [0-5.12]

L) Positive

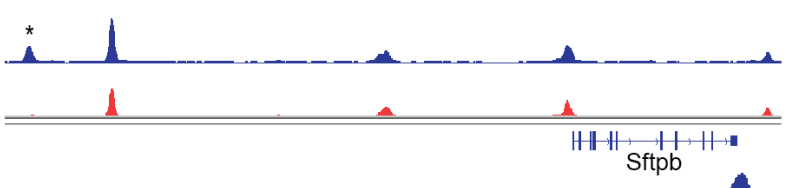

Negative 12kb; [0-1.20]

M) Positive Negative
19kb; $[0-0.84]$

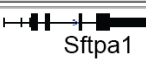

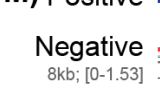

Figure 6: FoxA1/2 regulate global NKX2-1 binding in lung adenocarcinoma

A) Mean peak profile of FoxA1/2-positive and FoxA1/2-negative NKX2-1 ChIP peaks (fragments) over all AT1specific and 
B) AT2-specific NKX2-1 binding sites as defined by NKX2-1 ChIPseq in healthy adult mouse AT1 and AT2 lung cells $^{23}$. Representative of four biological replicates of NKX2-1 ChIPseq in FoxA1/2-positive and FoxA1/2negative LUAD organoids (1027B).

C) Heatmap representing NKX2-1 peak fragments of 2,758 differential NKX2-1 binding sites as determined by MAnorm2 in FoxA1/2-positive (left panels) and FoxA1/2-negative (right panels) LUAD organoids. 1,262 peaks enriched in a FoxA1/2-positive context (top panels) and 1,496 enriched in a FoxA1/2-negative context (bottom panels) with a significance cutoff of padj $<0.05$.

D) Select transcription factor motifs detected in NKX2-1 peaks enriched in FoxA1/2-positive or...

E) FoxA1/2-negative organoids.

F) Select transcription factor motifs for those NKX2-1 peaks not called as significantly enriched in either condition.

G) Graphical representation of significant association between differential NKX2-1 binding and differential gene expression ( $p<0.0001$, Fishers exact test). Differential NKX2-1 peaks were assigned to protein-coding genes and then intersected with genes significantly changed after Foxa1/2 deletion as determined by RNAseq of two biological replicates of isogenic 1027B organoids. $25.7 \%(170 / 662)$ of genes enriched in FoxA1/2positive organoids are associated with a FoxA1/2-positive enriched NKX2-1 binding site. 8.6\% (57/662) of genes enriched in FoxA1/2-positive organoids are associated with a FoxA1/2-negative enriched NKX2-1 binding site. 7.6\% (30/396) of genes enriched in FoxA1/2-negative organoids are associated with a FoxA1/2positive enriched NKX2-1 binding site. 18.0\% (71/396) of genes enriched in FoxA1/2-negative organoids are associated with a FoxA1/2-negative enriched NKX2-1 binding site.

H) NKX2-1 ChIP peaks in FoxA1/2-positive and FoxA1/2-negative organoids for AT1 marker Pdpn and AT2 markers Lamp3 (I), Sftpa1 (J), Sftpb (K), Sftpc (L), and Napsa (M). Scale for each gene indicated in individual figures. 


\section{SUPPLEMENTAL FIGURE 6}

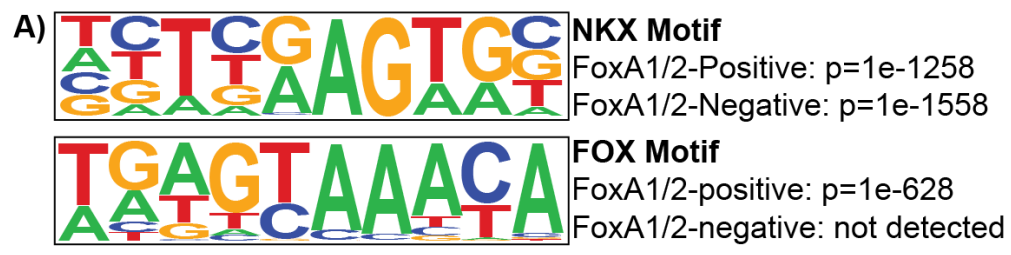

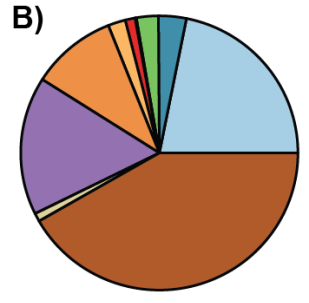

All Peaks

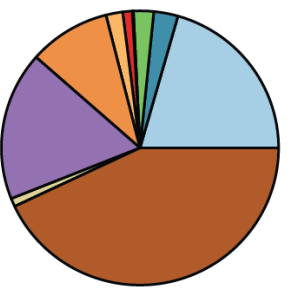

FoxA1/2-Positive Peaks

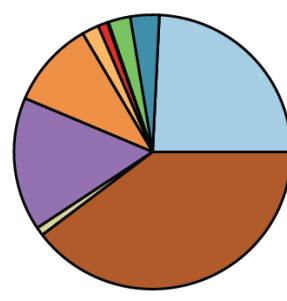

FoxA1/2-Negative Peaks

C) Lung Epithelial Lineage NKX2-1 Binding Sites

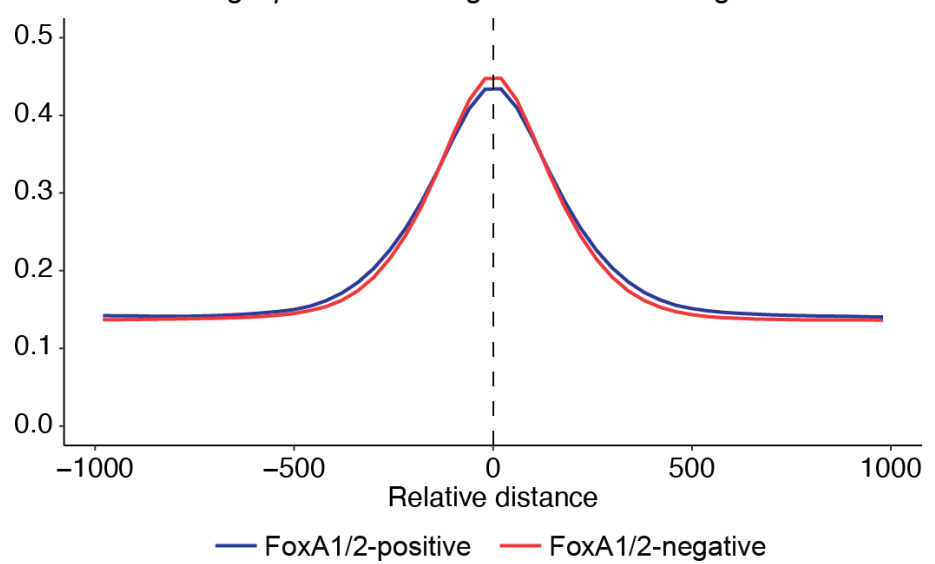

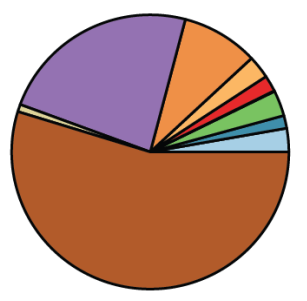

FoxA1/2-Positive Enriched Peaks

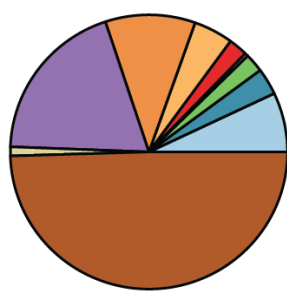

FoxA1/2-Negative Enriched Peaks $\square$ Promoter (<=1 kb)

$\square$ Promoter (1-2kb)

$\square$ Promoter (2-3kb)

$\square$ 5' UTR

$\square$ 3' UTR

$\square$ Other Exon

$\square$ 1st Intron

$\square$ Other Intron

$\square$ Downstream (<=300)

$\square$ Distal Intergenic
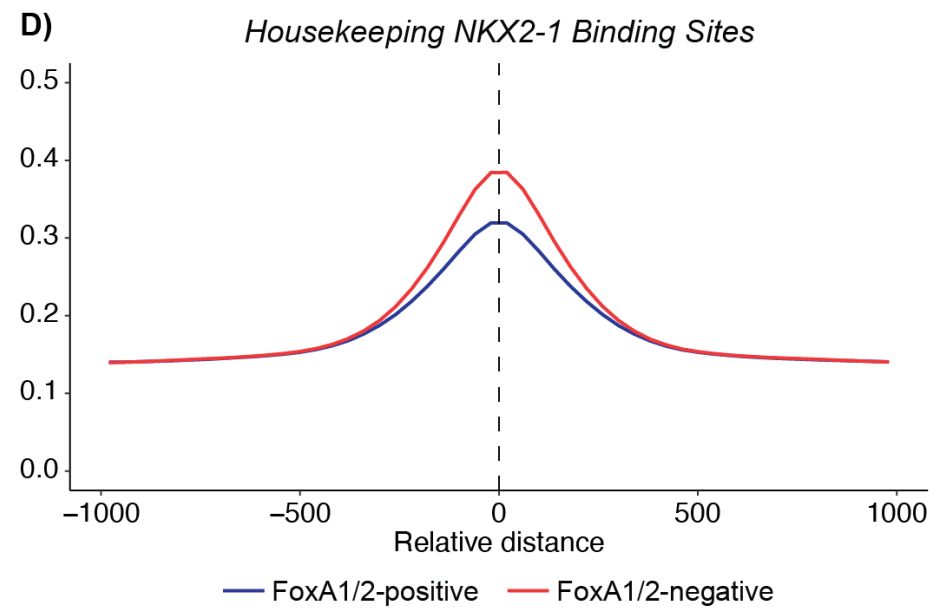

\section{Supplemental Figure 6, related to Figure 6}

A) NKX and FOX transcription factor motifs and their corresponding p-values in FoxA1/2-positive and FoxA1/2negative NKX2-1 ChIP peaks.

B) Genomic occupancy of all NKX2-1 ChIP peaks, FoxA1/2-positive NKX2-1 ChIP peaks, FoxA1/2-negative NKX2-1 ChIP peaks, NKX2-1 ChIP peaks enriched in FoxA1/2-positive cells, and NKX2-1 ChIP peaks enriched in FoxA1/2-negative cells.

C) Mean peak profile of FoxA1/2-positive and FoxA1/2-negative NKX2-1 ChIP peaks (fragments) over all lung epithelial lineage and...

D) Housekeeping NKX2-1 binding sites as defined by NKX2-1 ChIPseq in healthy adult mouse AT1 and AT2 lung cells ${ }^{23}$. 


\section{FIGURE 7}
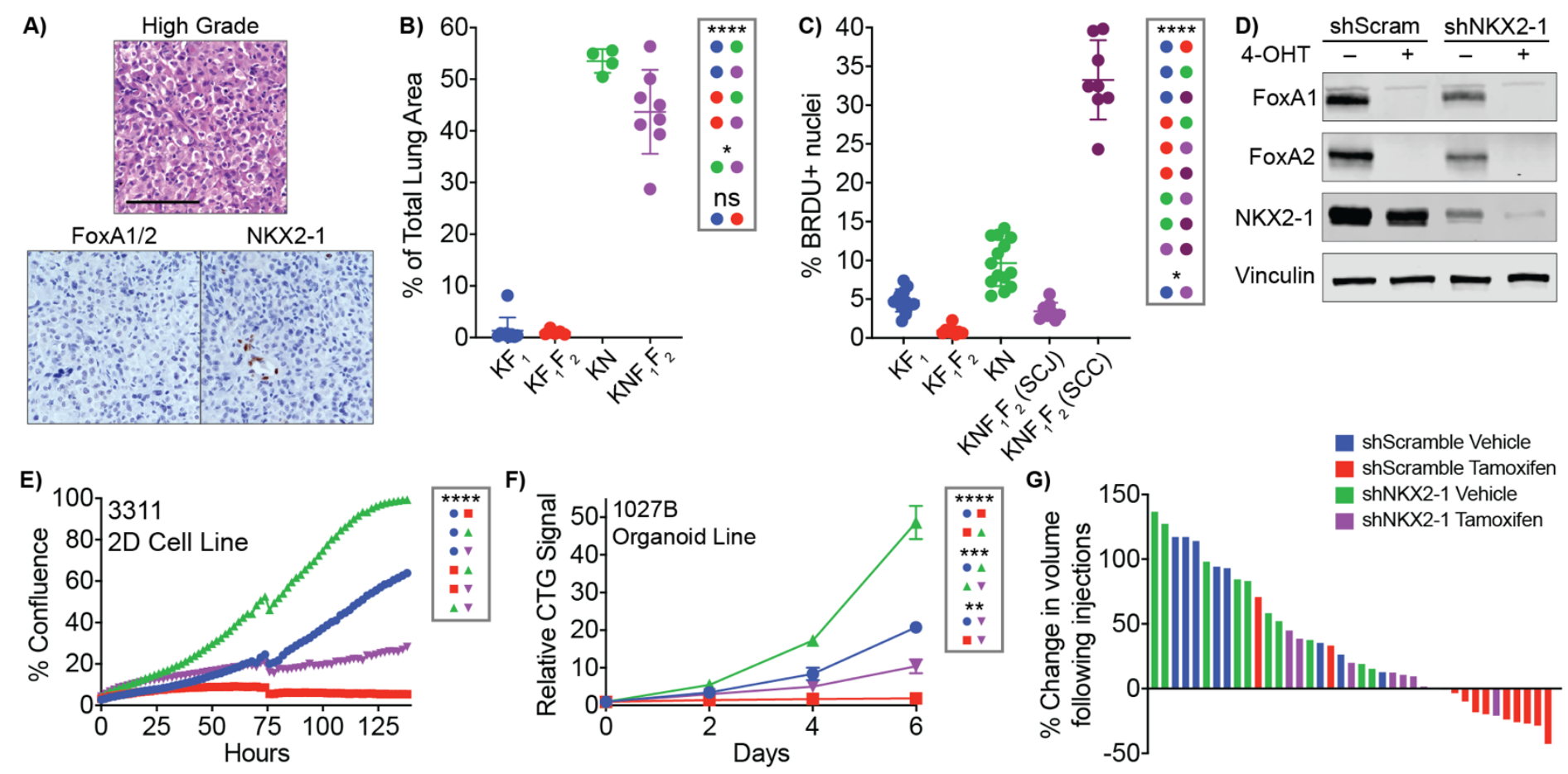

H)
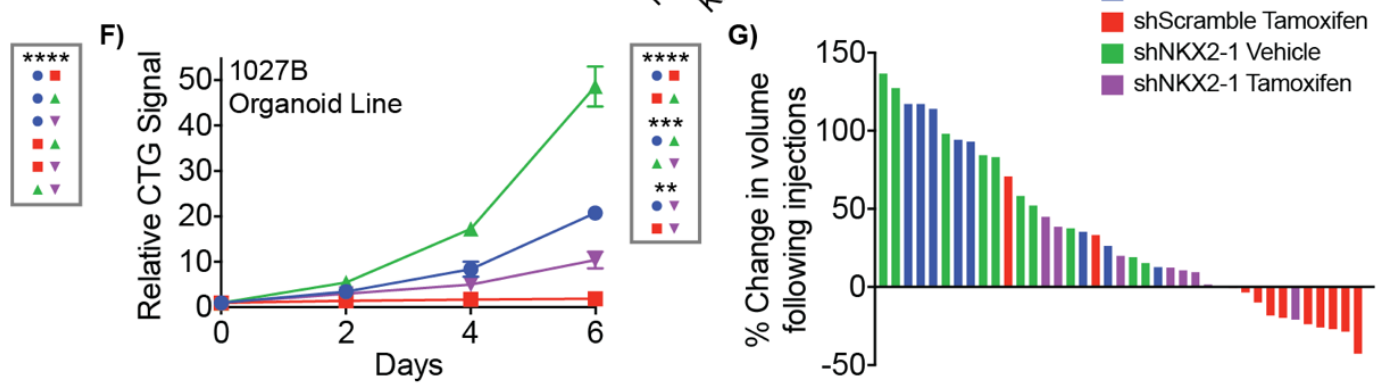

Pro-tumorigenic Cell Fates

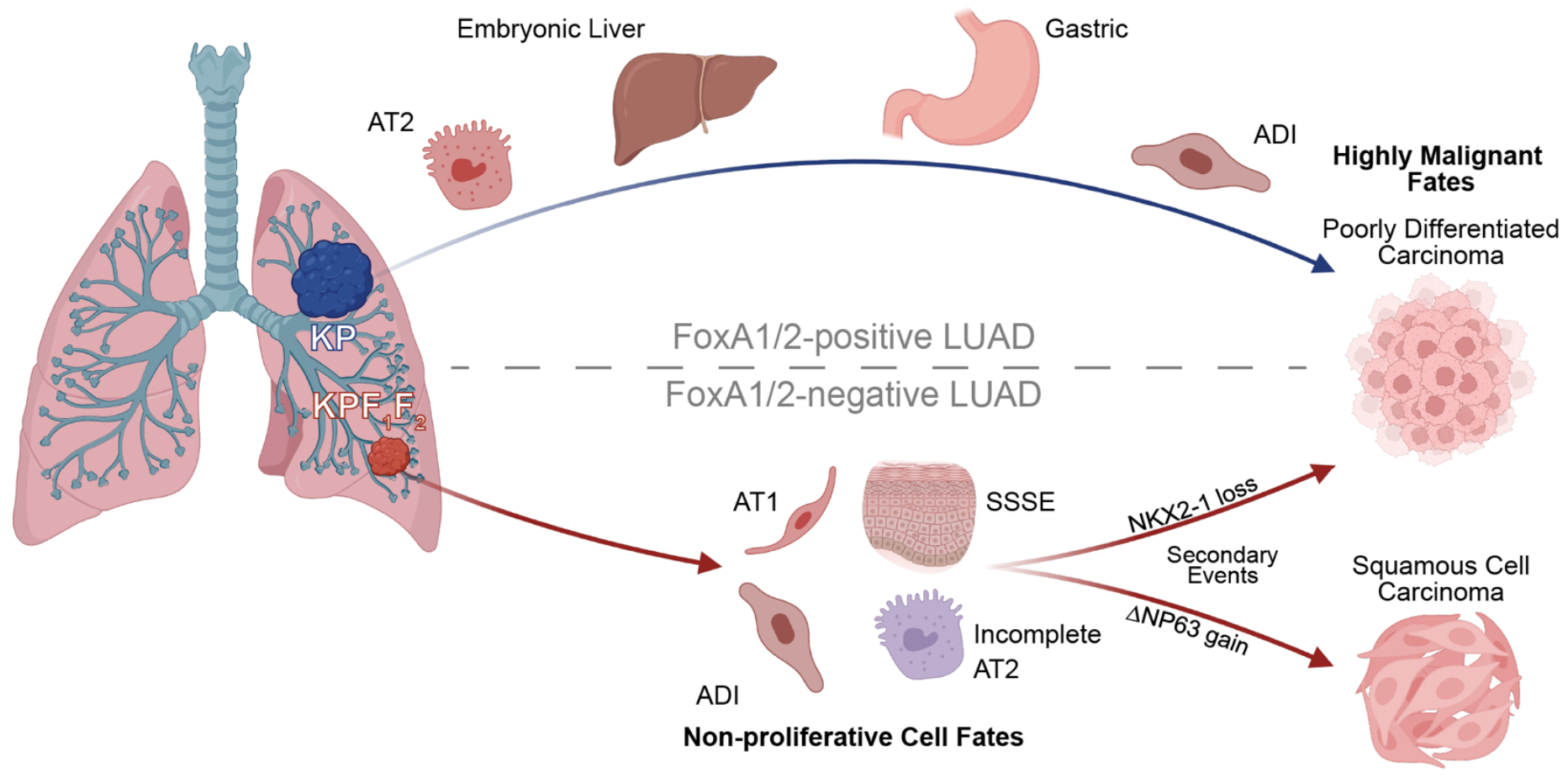

Figure 7: NKX2-1 restrains tumor growth in the absence of FoxA1/2

A) Representative images of high grade, NKX2-1-negative tumors that emerged in the FoxA1/2-negative cohort of long-term survival studies. Top panel H\&E, bottom panels IHC (scale bar $250 \mu \mathrm{m}$ ).

B) Tumor burden quantification of $\mathrm{KF}_{1}, \mathrm{KF}_{1} \mathrm{~F}_{2}, \mathrm{KN}$, and $\mathrm{KNF}_{1} \mathrm{~F}_{2}$ tumors initiated with Ad5mSPC-Flp, injected with tamoxifen 6 weeks later, and sacrificed at 12 weeks (multiple unpaired t-tests; ${ }^{*} p=0.0424 ;{ }^{* * * *} p<0.0001$ ). 
C) BRDU IHC quantification experiment shown in B (multiple unpaired t-tests; ${ }^{*} p=0.0366$; ${ }^{* * *} p<0.0001$ )

D) Western blot of protein extract from standard culture $\mathrm{KPF}_{1} \mathrm{~F}_{2}$ cell line (3311) transduced with shScramble and shNKX2-1 collected 3 days post 48-hour 4-OHT/vehicle treatment demonstrating successful knock down of NKX2-1.

E) Incucyte-based confluency growth assay of cells shown in D one week after 4-OHT/vehicle treatment. One representative experiment shown of two biological replicates with 8 technical replicates each (multiple unpaired t-tests of endpoint values; ${ }^{* * *} p<0.0001$ for all comparisons).

F) Timecourse experiment using the CellTiter-Glo 3D cell viability assay of cells shown in Figure 5A. CTG assay was performed on the day of seeding, as well as 2,4 , and 6 days post-seeding. Data shown relative to day 0 luminescence reads for each condition. One representative experiment shown of two biological replicates with 3 technical replicates each (multiple unpaired t-tests of endpoint values; ${ }^{* *} p=0.0016 ;{ }^{* * *} p<0.0005$; $\left.{ }^{* * * *} p<0.0001\right)$

G) Waterfall plot showing the change in individual 3311 subcutaneous tumor volumes. Percent change representing the first measurement after completion of tamoxifen/vehicle injections relative to the measurement immediately preceding treatment.

H) FoxA1/2 are required for the activation of the dual identity, combined pulmonary and gastro-intestinal identity that is a key step in the malignant evolution of this disease. In the absence of FoxA1/2, LUAD tumors shed a portion of their AT2 identity while activating an AT1-like identity and maintaining a PATS/ADI pulmonary state. Discrete subpopulations within both of these pulmonary states also express gene sets associated specifically with the superficial layers of the stratified squamous epithelium. We have shown that NKX2-1 acts in a tumor-suppressive manner in a FoxA1/2-negative context. In the absence of FoxA1/2 NKX2-1 localization dynamically changes, and these alterations are correlated with global gene expression changes. We have shown that NKX2-1 transcriptional activity is at least partially responsible for the gene expression changes following FoxA1/2 deletion, including both the emergence of AT1 and SSSE identities as well as a decrease in AT2 identity. We speculate that this transition to non-proliferative cellular identities upon Foxa1/2 deletion, as aided by de novo NKX2-1 activity, plays a major role in the growth inhibition observed in FoxA1/2negative LUAD. Over time, FoxA1/2-negative lesions can overcome this growth inhibition by either 
bioRxiv preprint doi: https://doi.org/10.1101/2021.06.29.450247; this version posted November 10, 2021. The copyright holder for this preprint (which was not certified by peer review) is the author/funder. All rights reserved. No reuse allowed without permission.

progressing into a fully malignant, keratinized squamous tumor or stochastically downregulating NKX2-1 expression and transforming into a high grade, poorly-differentiated tumor. 


\section{SUPPLEMENTAL FIGURE 7}
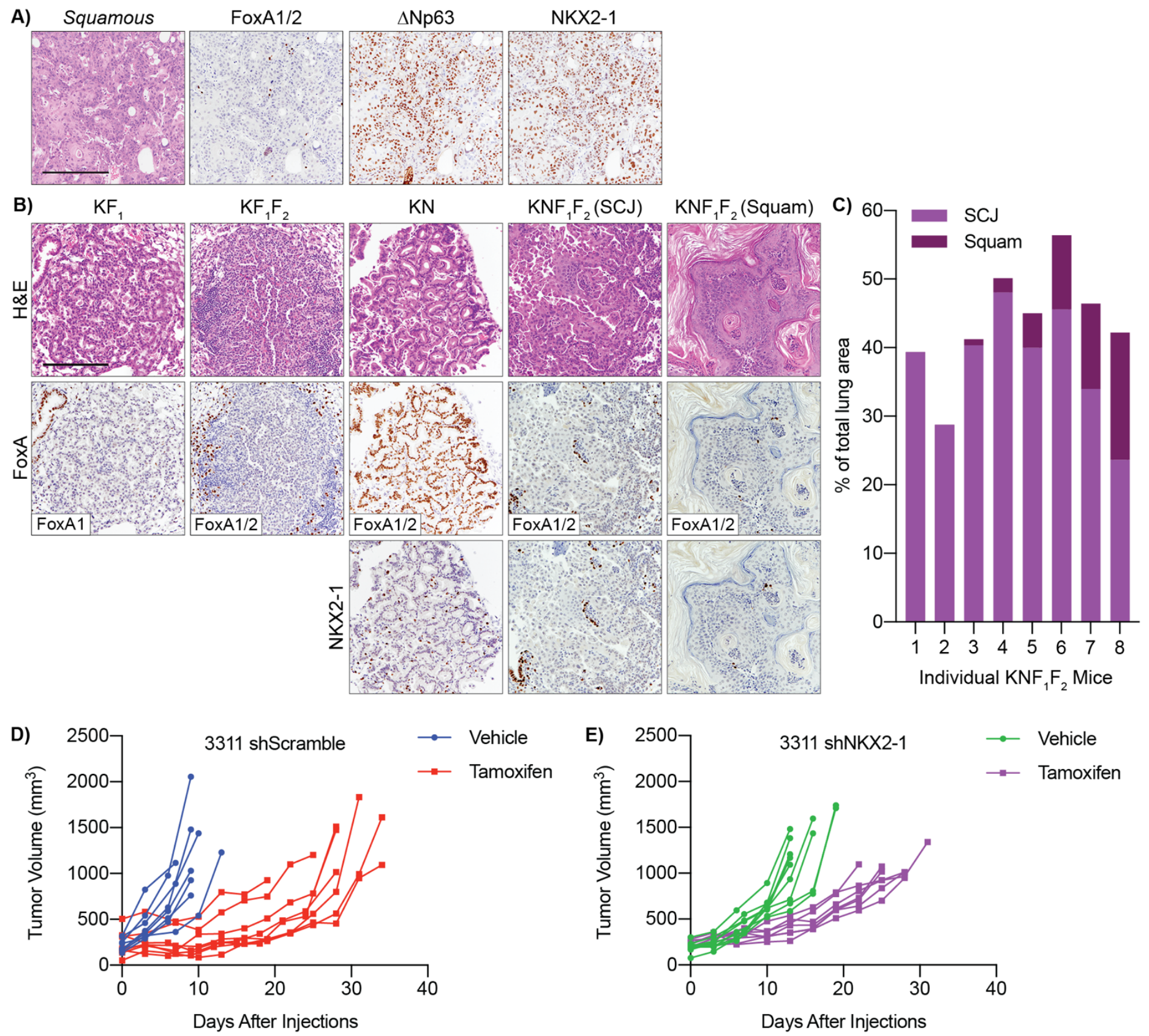

\section{Supplemental Figure 7, related to Figure 7}

A) Representative images of squamous, $\Delta$ Np63-positive, NKX2-1-positive tumors that emerged in the FoxA1/2negative cohort of long term KP survival studies (scale bar $500 \mu \mathrm{m}$ ).

B) Representative images of $\mathrm{KF}_{1}, \mathrm{KF}_{1} \mathrm{~F}_{2}, \mathrm{KN}$, and $\mathrm{KNF}_{1} \mathrm{~F}_{2}$ tumors in which alleles were deleted via tamoxifen treatment 6 weeks post-tumor initiation, and tissue was collected 12 weeks post-tumor initiation (SCJ=Squamocolumnar Junction; Squam=Squamous; scale bar $500 \mu \mathrm{m}$ ).

C) Percentage of tumor burden in individual $\mathrm{KNF}_{1} \mathrm{~F}_{2}$ mice attributable to $\mathrm{SCJ}$ and Squamous phenotypes. 
bioRxiv preprint doi: https://doi.org/10.1101/2021.06.29.450247; this version posted November 10, 2021. The copyright holder for this preprint (which was not certified by peer review) is the author/funder. All rights reserved. No reuse allowed without permission.

D) Measurements of subcutaneous tumor volume for individual mice allografted with 3311 shScramble and injected with vehicle or tamoxifen. Each line represents an individual tumor.

E) Measurements of subcutaneous tumor volume for individual mice allografted with 3311 shNKX2-1 and injected with vehicle or tamoxifen. Each line represents an individual tumor. 